Advances in Physics

Vol. 00, No. 00, Month-Month 200x, 1-91

\title{
Exactly solvable model of avalanches dynamics for Barkhausen crackling noise
}

\author{
Francesca Colaiori, SMC, INFM-CNR and Dipartimento di Fisica, "La Sapienza" \\ Università di Roma, P.le Aldo Moro 2, I-00185 Roma, Italy \\ (Received 00 Month 200x; In final form 00 Month 200x)
}

\begin{abstract}
We review the present state of understanding of the Barkhausen effect in soft ferromagnetic materials. Barkhausen noise (BN) is generated by the discontinuous motion of magnetic domains as they interact () with impurities and defects. BN is one of the very many examples of crackling noise, arising in a variety of contexts with remarkably similar features, and occurring when a system responds in a - jerky manner to a smooth external forcing. Among all crackling system, we focus on BN, where a I complete and consistent picture emerges thanks to an exactly solvable model of avalanches dynamics, known as ABBM model, which ultimately describes the system in terms of a Langevin equation for the velocity of the avalanche front. Despite its simplicity the ABBM model is able to accurately Treproduce the phenomenology observed in the experiments on a large class of magnetic materials, $\downarrow$ as long as universal properties are involved. To complete the picture and to understand the longUstanding discrepancy between the ABBM theory and the experiments - which otherwise agree Eexceptionally well - consisting in the puzzling asymmetric shape of the noise pulses, microscopic details must be taken into account, namely the effects of eddy currents retardation. These effects I can be incorporated in the model, and result, to a first order approximation, in a negative effective mass associated with the wall. The progress made in understanding $\mathrm{BN}$ is potentially relevant for other crackling systems: on one hand, the ABBM model turns out to be a paradigmatic model for the universal behavior of avalanches dynamics; on the other hand, the microscopic explanation of the asymmetry in the noise pulses suggests that inertial effects may also be at the origin of pulses asymmetry observed in other crackling systems.
\end{abstract}

\section{Introduction}

\section{(1.1 Crackling noise}

it

The term "crackling noise" $[1,2]$ refers to the signal that some disordered systems produce as a response to an external driving field smoothly changing in time. Due to the presence of disorder, crackling signals are extremely irregular, despite the steady increase of the external forcing. They are typically characterized by a sequence of pulses of very different sizes and durations, separated by quiescence intervals. Tiny events occur very frequently, while large ones are Jorare, with power laws probability distributions.

In this review we focus on one particular case of noise, the so called Barkhausen noise $(\mathrm{BN})$, which is emitted by ferromagnetic materials during the magnetization reversal process [3-5]. 
Systems that "crackle" are found in many different situations, and, remarkably, the corresponding signals often share some common characteristic features. Examples of crackling signals, besides BN, include the shear response of a granular media [6-8], the acoustic emission during martensitic phase transitions [9], the bursts of dislocations activity in plastic deformation [10-14], the dynamic of superconductors [15-17] and superfluids [18,19], the fluctuations in the stock market [20-22], the dielectric polarization of ferroelectric materials [23], the acoustic emission in fractures [24-28], and the seismic activity in earthquakes $[29,30]$.

Crackling noise signals are expected to encode information on the physical process that generates them. Understanding the statistical properties of these jerky emissions, is therefore a step towards the understanding of the microscopic dynamics taking place in the system that crackles. Moreover, the fact that very diverse systems behave in a remarkably similar manner, suggests that some general basic principle may exist in the underlying physics. If this is the case, then, understanding in detail one of them, may give insight to others, and propel other fields of research.

BN can be seen as a case study, for which a satisfying picture is finally available. A main ingredient in this picture is a model of avalanche dynamics known as ABBM model, named after the authors of the original papers, Bruno Alessandro, Cinzia Beatrice, Giorgio Bertotti and Arianna Montorsi, whose companion seminal papers $[31,32]$, one devoted to the developing the theory, the other to the comparison with experiments, have represented a huge step forward in the understanding of BN. The ABBM model, corresponds to a mean field description of an elastic magnetic wall moving in a disordered ferromagnet under the effect of an external driving field. The system is ultimately described by a simple Langevin equation for the velocity of the center of mass of the wall.

Despite its simplicity, the ABBM model is able to reproduce with striking accuracy most of the phenomenology observed in $\mathrm{BN}$ experiments on a large class of magnetic materials. Moreover, thanks to a mapping onto a simple stochastic process, all the results concerning the noise statistics can be derived analytically, and have a clear and direct interpretation. In particular, this simple mean field description allows to explain the origin of the power law distributions of size and duration of pulses, both in the quasi-static limit, where the applied field variations are extremely slow, and at finite driving field rates. In this second case, the model is also able to predict how these distributions depend on the driving field rate.

A more refined analysis of the experimental data against theoretical predictions includes the comparison of the shape of the avalanches. This is where the ABBM model unexpectedly fails, being unable to predict the characteristic leftward asymmetric form of BN pulses. The origin of this asymmetry lies 
in the non-instantaneous response of the eddy fields to the domain wall displacement. To understand and evaluate the effect of such delay, one as to take into account the dynamical effect of eddy currents in the Maxwell equations for the eddy field. Eddy currents retardation gives rise to an "anti-inertial" effect, that can be accounted for, to the first order, by associating a negative effective mass to the wall.

Once the effective mass is identified, the corresponding inertial term can be incorporated into the ABBM equation of motion. The modified ABBM model that comes out from this analysis gives a complete picture of the Barkhausen effect, consistent with the phenomenological observations: it is still successful in reproducing the distribution of sizes and durations of pulses, and also correctly reproduces the asymmetry in their shape.

It is interesting to underline the role of universality versus non- universality in the theory of Barkhausen effect. The standard statistical mechanics approach usually focuses on universal quantities. Definitely, universality is a key and extremely powerful concept at the basis of this approach, as it allows to predict the essential behavior of self-similar systems by means of very simple models. This consideration indeed applies to the case of Barkhausen noise, where, in analogy to critical phenomena, most of the statistical properties of the signals only depend on general properties of the system, while they are independent of the microscopic details: different magnetic samples respond to the forcing by an external field by producing events of magnetization reversal characterized by the same power law distributions, regardless of the specific microscopic structure of the material.

However, some interesting features of $\mathrm{BN}$ turn out to be of microscopic origin. Although, consistently with universality, microscopic details would become negligible on extremely large scales, they have an unusually large effect in Barkhausen signals, an effect that is still significant at the experimental scale. On one hand this can be seen as an inconvenient and a limitation of the methods of statistical mechanics, since it means that not all the relevant aspects of the phenomenon can be captured by simple models. On the other hand, the identification of some macroscopic effect of the microscopic details, provides a mean to extract information on microscopic quantities by Barkhausen measurements. The asymmetry of the pulses gives indeed a measure of this type. As an example, the skewness of the pulses measured as a function of the pulse duration shows a peak, that allows to identify a characteristic timescale for relaxation, which corresponds to the ratio between mass and damping constant.

In this review we will deal with both these aspects of the theory, in the attempt to put together a number of results achieved in recent years. We will also try to underline which points are still unclear and would need further analysis. 


\section{$1.2 \quad$ Outline}

The paper is organized as follows. Section 2 reviews some general facts about Barkhausen noise. We start with a short historical note (2.1). Then we discuss how magnetic materials are classified, in order to clarify to which ones the following theory applies (2.2). We shortly describe the typical set-up and the problems arising in $\mathrm{BN}$ experiments in subsection 2.3. BN phenomenology, as it comes out from experiments, is described in subsection 2.4. In subsection 2.5 we discuss the two main theoretical approaches to BN that have been developed in recent times: one based on a microscopic description in terms of a random field Ising model, the other describing the magnetization process in terms of the dynamic of magnetic interfaces, that will be further developed in the next section.

In section 3 we focus on the second approach: first we introduce the ABBM as a phenomenological model (3.1) and underline its main limitations (3.2). To put the model in a more general framework we then briefly discuss the problem of the dynamic of a generic elastic interface in a random media (3.3), and then we specialize to the case of a magnetic interface (3.4). The purpose of this digression is to recover the ABBM model as a mean field for a model of interface dynamic (3.5), therefore corroborating its validity.

Section 4 is the core part of the paper: we show how the ABBM equation is mapped onto a Langevin equation describing a biased random walk in logarithmic potential with absorbing boundary conditions at the origin (4.1). This approach is amenable of analytical treatment and allows to work out exactly a number of properties, and to give a direct interpretation of the phenomenology observed in the experiments in terms of properties of the corresponding stochastic process (4.2). The power law distributions in avalanches' sizes and durations can be exactly calculated in terms of distributions of return times to the origin (4.3). The continuous dependence of the exponents in the power law distributions on the driving field rate can also be calculated, and turns out to be related to the marginality of the logarithmic perturbation to the free random walk (4.4). The existence of a threshold in the driving field rate can be derived from recurrence properties of a free random walk (4.5), and the existence of a cut-off in the power law distributions can be associated to the bias term (4.6). The average shape of the Barkhausen pulse can be derived in terms of the excursion of the process (4.7), and finally the power spectra can be derived from the process correlations (4.8). In subsection 4.9 we summarize the ABBM predictions and compare them with the phenomenological observations. In the last subsection (4.10) we discuss the scaling of the cut-offs with the demagnetizing factor. This scaling is not entirely captured by the mean field approximation, although it is predicted by the full elastic interface model in $d=3$. 
Section 4 collects a number of results, most of which, but not all, are spread in literature, but to which has never been given a comprehensive exposition in the BN framework before. Some of these results have been indeed developed in an unrelated context, to deal with a completely different problem [33]. For this reason in this part we made an effort to give a self-contained exposition.

The issue of the asymmetry of Barkhausen pulses (that the ABBM model is not able to reproduce) is treated in section 5, a characteristic leftward asymmetry in the average pulse shape is observed in experiments (5.1), which is due to the non-instantaneous response of the eddy currents field to the wall displacement (5.2). The eddy current retardation gives rise to inertial effects, that are accounted for in a generalized ABBM model by associating a negative effective mass to the domain wall (5.3). In subsection 5.4 we discuss how this non-universal effect is affected by the sample geometry, and, finally, in subsection 5.5 we compare theory predictions and experiments.

In section 6 we discuss some other crackling systems that may be tackled with an approach similar to the one used for the Barkhausen effect: the case of the dynamic of a granular media under shear (6.1), and the case of seismic activity during earthquakes (6.2).

Section 7 and 8 are devoted to conclusions and acknowledgments respectively.

\section{The Barkhausen effect: history, experiments and theoretical approaches}

\section{$2.1 \quad$ Short historical note}

Barkhausen noise is probably one of the first crackling signals ever recorded. Indeed the Barkhausen effect has been known for almost a century: its first observation dates back in 1919, when Heinrich Barkhausen noticed that "iron produces a noise when magnetized: as the magnetomotive force is smoothly varied $[. .$.$] it generates irregular induction pulses in a coil wound around the$ sample that can be heard as a noise in a telephone" [3].

Barkhausen's first experiment consisted in observing the variations of the magnetization in a ferromagnetic sample subject to a slowly and smoothly changing magnetic field. A pickup coil winded around the sample was used to detect the variations of the magnetization as schematically shown in figure 1. As the magnetization reverses, the variations of the magnetic flux induce a voltage in the coil, that indirectly measures the changes in the magnetization of the sample, and that may eventually be translated in real noise through an amplifier. The signal recorded in correspondence to jumps in the magnetization, appears to be very irregular, no matter how smooth is the variation of the external field. 


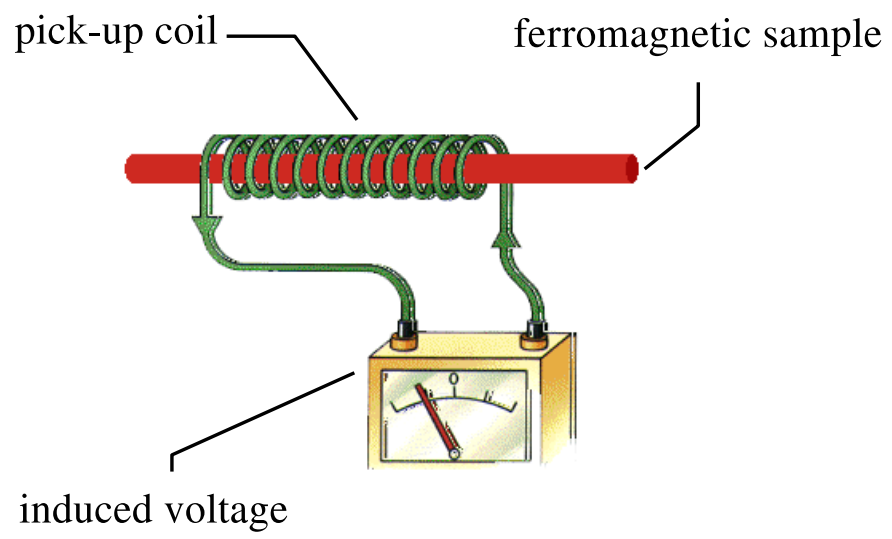

Figure 1. Sketch of experimental set-up used in the first BN experiment. The bar is the ferromagnetic sample, which is inserted in the pick-up coil. As the external field is changed, an electromotive force is induced in the coil. The voltage can eventually be transformed in real noise through an amplifier.

Nowadays, magneto-optical methods are also available, that allows a direct observations of moving domains, however, the most reliable experiments in terms of signal statistics are still based on inductive measurements. In order to be capable to record clean signals with reduced background noise and high statistic, modern experimental settings make use of sophisticated equipments, however, the basic set-up for inductive BN experiments is conceptually still the same as the one shown in figure 1 .

Barkhausen wondered about what could be the source of the signal emitted during the magnetization of the iron sample, and he mistakenly concluded that the sequence of pulses was generated by a corresponding sequence of sudden and complete reversals of entire magnetic domains. At that time, his experiment was indeed taken as the first indirect observation of magnetic domains, whose existence had been postulated a few years before (1907) by Weiss [34]. Almost 20 years later (1938) Elmore was able to perform an experiment on a cobalt crystal where the motion of domain boundaries was directly observed and measured for the first time [35]. However Elmore did not recognize this motion as the source of BN. Only more then 10 years later (1949), after another experiment by Williams and Shockley on a FeSi crystal [36], the origin of $\mathrm{BN}$ was finally ascribed to the correct cause, namely to the irregular fluctuations of magnetic domain boundaries, rather then to the sudden inversion of domains. This fact was definitely clarified in the same year by Kittle in his fundamental review on the physical theory of ferromagnetic domains [37]. A 
nice exposition of the first steps in the understanding and misunderstandings of Barkhausen effect is given in a recent review by G. Durin and S. Zapperi [5], which also includes in appendix a translation from German to English of the original paper by H. Barkhausen.

Since its first observation, a lot of work has been done in order to "decode" the Barkhausen signal, both on the experimental side and on the theoretical one. For a long time, theoretical studies have been based on a phenomenological approach, which described the signal as a superposition of random elementary jumps. Then, it became clear that BN could represent a powerful tool to investigate the magnetization process and the hysteretic properties on a microscopic scale, and much effort has been devoted in developing physically grounded models that could allow to relate the phenomenology to its microscopic origin. The theoretical studies have followed two kind of approaches: one based on a microscopic description in terms of spins in a random magnetic field (see section 2.5); the other one, which is the one on which we will focus in this review, describes the system in terms of a fluctuating magnetic interface that moves under the action of the external field.

The research on BN has been in part motivated, especially at the beginning, by the applications of Barkhausen effect to material testing. Barkhausen emissions are indeed commonly used to check in a non-destructive way the integrity of magnetic samples: the signal intensity is sensitive to the changes in material microstructure, and to the presence of residual stresses (in magnetostrictive positive materials compressive stresses will decrease the intensity of Barkhausen noise while tensile stresses will increase it). These properties make $\mathrm{BN}$ an efficient tool for the detection of micro-imperfections and for the evaluation and mapping of the local distribution of residual stresses [38-45].

Most of the recent research on the statistical properties of $\mathrm{BN}$ is however theoretically oriented, and primarily aimed to gain understanding on the hysteretic properties of ferromagnetic materials, and to investigate the magnetization reversal process on a microscopic scale.

Recently, further interest has been raised by the fact that many other driven dissipative systems have been found to respond to a smooth forcing in a disordered manner, with very similar and reproducible statistical features. To this kind of response has been given the general denomination of "crackling noise". Clearly, the irregular response is due to the presence of disorder in the system, and the challenge is to get information on the specific system under study by decoding the noise that it generates. As the recent encouraging advances in the understanding of $\mathrm{BN}$ compose a rather satisfying picture of the phenomenon, $\mathrm{BN}$ emerges as an attractive case study to understand crackling noise in general. 


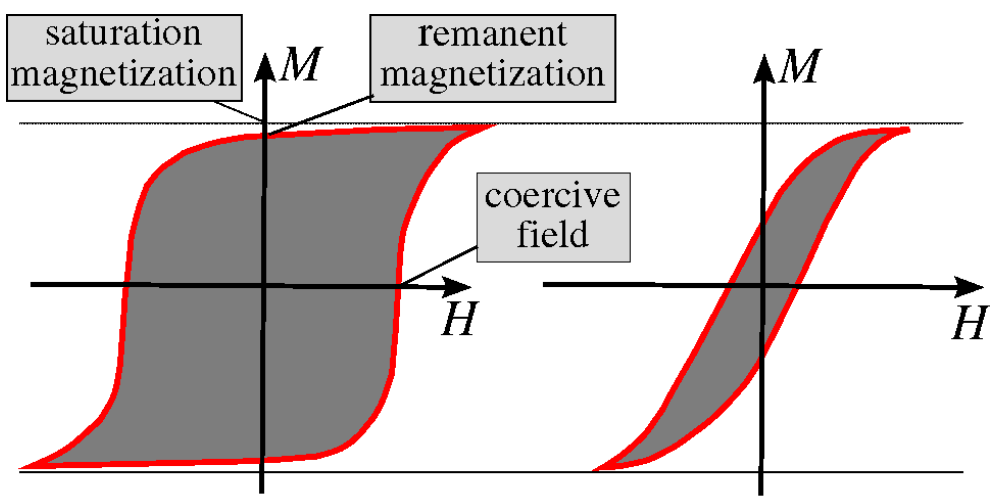

(a)

(b)

Figure 2. Sketch of a typical hysteresis loop for a hard magnet (a) and a soft magnet (b).

\section{$2.2 \quad$ Soft magnets versus hard magnets}

The understanding of hysteretic properties of magnets has an interesting overlap with material science, and the theory that we describe has been built always keeping close contact with the experimental side. Therefore, although the aim of this review is mainly theoretical, we believe that, in order to put in a correct framework the theory, understand its limitations, and to keep close the connection with the experimental counterpart, it is worth to discuss briefly how magnetic materials are classified, and to clarify to which ones this theory applies, and to which ones it does not.

Magnetic materials are roughly classified in soft and hard. Soft magnets are those characterized by very tiny hysteresis loop, reflecting the fact that they are easily magnetized and demagnetized by applying relatively small fields. Hard magnets are the exact opposite: they have very wide and squared hysteresis loops, and they need a big effort in terms of applied field in order to be magnetized and demagnetized. The two classes of magnets are quite well

separated, and there is not much in between. A good quantitative measure of "magnetic hardness", is given by the coercive field, which is the field needed to bring back to zero the magnetization of a saturated sample. This quantity differs, between soft and hard magnets, by several orders of magnitudes (see figure 2) [46].

The markedly different hysteretic behavior observed in the two classes of materials is due to the fact that the magnetization reversal proceeds by different mechanisms in the two cases. In soft magnets it is dominated, except than close to saturation, by the nucleation and displacement of magnetic walls. This is a process with low energetic cost, that translates into a small loop area, which measures the dissipated energy. On the other side, in hard magnets the wall nucleation and/or displacement is strongly impeded, or totally 
suppressed, and magnetization reversal by coherent spin rotation enters into play.

Hard magnets have highly anisotropic magnetic properties. They are usually composed from alloys containing rare earth elements (typical ones are $\mathrm{Co}_{5} \mathrm{Sm}$ and $\mathrm{Ne}_{2} \mathrm{Fe}_{14} \mathrm{~B}$ ). In single domain particle magnets the nucleation of domains is prevented by building samples made out of very small magnetic grains, whose size is comparable with the domain wall thickness, and each one with a random easy magnetization direction. Due to the high anisotropy, the magnetization can only reverse by coherent spin rotation when the applied field is large enough to force the spins out of the easy magnetization direction. Due to the high coercive field, hard magnets cannot be accidentally demagnetized when subjected to small fields. Also, they have a remanent magnetization very close to the saturation value, meaning that they are able to retain their magnetization when the applied field is removed. These properties are extremely desirable in the design of magnetic storage devices.

Examples of soft materials are Iron-Silicon alloys, crystalline Iron-Nickel alloys (permalloys), and amorphous metallic alloys (typical is the $\mathrm{FeCoB}$ ). Applications of soft magnetic materials exploit the large flux changes obtained with a small change in the applied field, and the small energy loss. Soft magnets are used for example in transformers, motors, and inductors, and also as field sensors in magnetic recording devices. The magnetization reversal in these materials is dominated by the low-cost process of magnetic domain displacement. Domain walls, and the domain walls movement can be directly observed in experiments based on the magneto-optical Kerr effect (MOKE). Reflection of a beam of linearly polarized light from the surface of a magnetized sample causes the polarization to become elliptical, with the principal axis rotated with respect to the incoming light, depending on the magnetization direction. An analyzing filter in the reflected beam is then used to generate magnetic domain contrast.

The theory described in this review is based on a mesoscopic description in terms of dynamics of domain walls, therefore it only applies to soft magnets. The magnetization reversal process in hard magnets is indeed worth of attention, however, in this case, a microscopic description in terms of spin models would be more appropriate. As it will be discussed in subsection 2.4. soft materials have different statistical properties depending on the range of the interactions ruling the dynamics. The mean field theory which is the focus of this review applies to those with long range interactions.

\subsection{Barkhausen noise experiments}

In inductive experiments $\mathrm{BN}$ is detected by a pick-up coil wound around the ferromagnetic sample during the magnetization of the material, which takes 
place under the action of a varying external field $H(t)$. As the magnetization reverses in the sample, the variations of the magnetic flux $\dot{\Phi}$, induce an electromotive force $V$ in the pick-up coil, which has a contribution from the applied field across the pick-up coil, and another one given by the magnetic field inside the material:

$$
V=-N \dot{\Phi}=-N \mu_{0}\left(A_{c} \dot{H}+A \dot{M}\right)
$$

where $\mu_{0}$ is the vacuum magnetic permeability, $\mathrm{N}$ is the number of coil turns $A_{c}$ is the coil cross section, $A$ is the sample cross section, and $M$ the magnetization.

In high susceptibility materials the contribution coming from the first term in equation (11) can be neglected, since, as long as the susceptibility $\chi=$ $\left(\mu / \mu_{0}-1\right)$ is much larger then 1 (where $\mu$ is the magnetic permeability of the ferromagnetic material), the contribution from the magnetization rate $\dot{M}$ is much larger then the one due to the variation of the applied field $\dot{H}$. To neglect the first term in equation (11), it is also necessary that the sample cross section $A$ is not too small with respect to the pick-up coil section $A_{c}$.

In low susceptibility materials, or in cases where the condition $A \simeq A_{c}$ is not easy to satisfy, as for example in the case of thin films, it is possible to compensate the induced flux in air by using another pick-up coil with the same number of turns and cross section, but wound in the opposite direction.

With the eventual addition of the compensation coil, it can therefore always be assumed that the variation of the magnetic flux $\dot{\Phi}$ is approximately proportional to the rate of change of the magnetization:

$$
V \simeq-N \mu_{0} A \dot{M}
$$

In the simplest experimental conditions, where there is just one domain wall separating two regions of opposite magnetization, the rate of change of $M$ is twice the magnetization of the region spanned per unit time. The volume of this region is given by $v d L$ in the geometry and notations of figure 3, and where $v$ is the average wall displacement velocity. This volume, times the magnetization per unit volume $M_{s} / A L$, gives the magnetization rate

$$
\dot{M}=2 M_{s} v d / A
$$

where $M_{s}$ is the saturation magnetization.

Therefore, the induced voltage $V$ measured by the pick-up coil is just proportional to the wall velocity $v$ :

$$
V=-2 N \mu_{0} d M_{s} v
$$




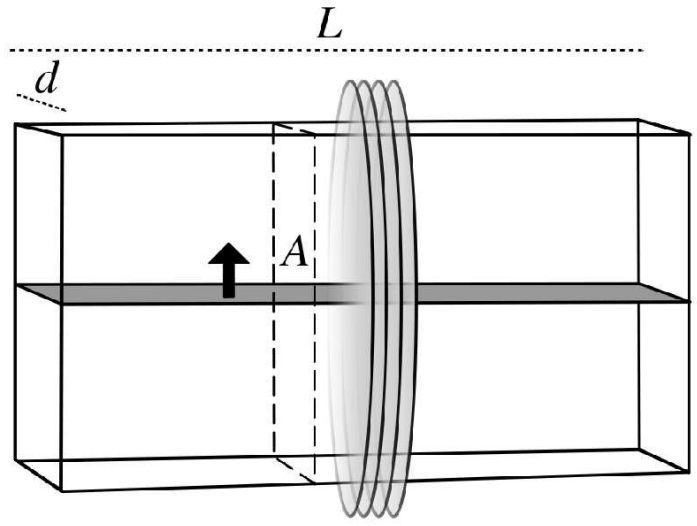

Figure 3. Sample geometry for a typical inductive BN experiment.

Note that the sample thickness $d$ in the direction perpendicular to the wall movement and to the coil axis is the only geometrical parameter that enters in the intensity of the BN signal.

The number of turns in the pick-up coil amplifies the signal by a factor $N$, thus one could be tempted to use wide detection coils. However, we stress that this is not a good choice: the magnetostatic field produces a counterfield which has a crucial role in the domain wall dynamics. This counterfield depends on sample geometry and domain structure, and it is homogeneous only in special geometries. However it is convenient to assume it to be constant also in more general cases. This is a good approximation only as long as one limits the region of the pick-up coil.

In the more complex case where several domain walls are present, the signal encodes the complex collective effect of many interacting domains. In this case the quantity $v$ in equation (4) approximately measure the velocity of the active walls.

Due to the presence of various types of disorder, like non-magnetic inclusions and dislocations, the wall movement is discontinuous, since the disorder locations act as pinning points. As the external field is smoothly increased, the magnetization changes in steps, in correspondence to jumps of the magnetic interface, or, in microscopic terms, in correspondence to avalanches of spin flips. The hysteresis loops is also discontinuous, and the signal, which is proportional to the derivative of the magnetization, plotted versus time, looks like a disordered series of pulses (see figure 4).

Note that, in identifying the Barkhausen signal with the derivative of the hysteresis curve, we are assuming that the applied field $H(t)$ is increased linearly in time: indeed, in BN measurements, often - but not always - a driving field with a triangular time profile is applied, instead of one with the sinusoidal 




Figure 4. Sketch of a typical hysteresis loop for a soft magnet. The loop is not smooth, rather the magnetization increases in jumps as shown in the zoom. The inset on the bottom right side is the derivative of the magnetization, proportional to the typical Barkhausen signal, detected from the induced voltage. The voltage directly measures the velocity of the domain wall: pulses correspond to jumps of the magnetic interface that gets repeatedly stuck by the various kind of impurities and imperfection, acting as pinning centers.

shape commonly used in hysteresis experiments. In this case, the variations of magnetization with respect to the applied field are directly translated in derivatives with respect to time.

There exists a vast literature reporting results and data about BN experiments in soft materials. However, not all the data collected are reliable when one is interested in statistically analyzing and modeling the self-similar properties of the noise, as the details of the experimental procedure may have a serious impact on the quality of the signal. Unfortunately, the need to establish a standard in the experimental set-up that could allow a rigorous comparison of the data, has been recognized as an important issue only in recent times.

The most relevant factor to take into account in order to ensure a reliable noise signal is to ascertain the stationarity during the dynamics. This question 


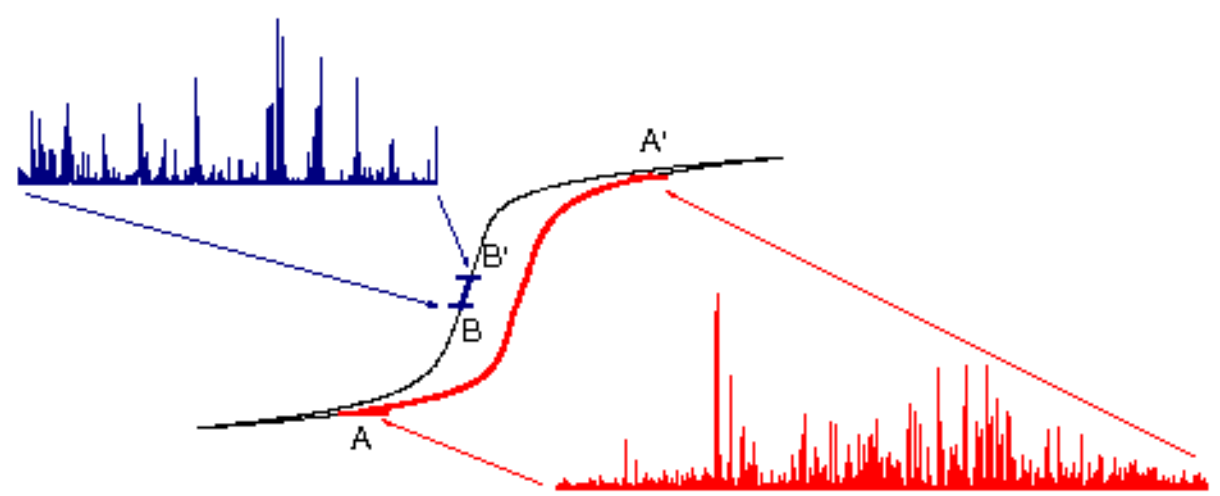

Figure 5. Hysteresis loop of a $F e_{85} B_{15}$ amorphous alloy under moderate tensile stress $(10 M P a)$. The signal measured along the whole half cycle (from $A$ to $A^{\prime}$ ) is highly non-stationary. The interval $B B^{\prime}$ represent the interval where the permeability can be assumed to be constant, and where the Barkhausen signal is stationary. (Reprinted with permission from [48]). [48] Copyright 2006 by Springer.

is crucial, since non-stationarity strongly biases the scaling of the avalanches distributions. However, it is not always easy to check whether the system is in a stationary state or not.

In the context of BN, the statistical properties of the signal strongly depend on the region of the hysteresis loop where the measure is recorded. This question was first raised by Bertotti and Fiorillo [47], who suggested to restrict the measures to the part of the loop corresponding to constant average permeability, where the condition of stationarity is guaranteed. This important point has however been disregarded in many experimental studies.

When collecting BN data, it is indeed crucial to take into account that the Barkhausen signal is stationary only in the linear region around the hysteresis loop, where the intensity of the applied field is close to the coercive field value (see figure 5). This is precisely the region where the domain wall displacement is the dominating - if not the unique - mechanism involved in the magnetization reversal process. Getting closer to saturation, other mechanisms enter into play, as the spins, which during the previous phase of the magnetization process stay parallel to the easy magnetization axis, start to coherently rotate in order to align to the external field (see figure 6).

Experimental studies that do not take this point into account, and average Barkhausen signals over the whole hysteresis loop, will mix the effect of the different magnetization mechanisms and of different dynamical conditions, making very difficult to attribute to the corresponding data a clear physical interpretation.

The role of stationarity has been recently further analyzed in great detail by Durin and Zapperi [48]. In their work they show both with simulations and experiments that in non-stationary conditions the distributions of avalanches, 



Figure 6. Typical magnetization loop for a soft magnet. On the right hand side the magnetization domain structures corresponding to five different regions of the hysteresis loop are sketched, assuming that only a single domain wall is present. The domain structure in (a) corresponds to a zero magnetization configuration. The domain wall divides the sample in two regions of opposite magnetization, oriented along the material easy magnetization directions. The two small triangular domains are the so called closure domains. Their presence is neglected in the following treatment. Increasing the applied field, the domain wall moves as in (b) in order to increase the size of the domain magnetized closer to the direction of the applied field. In this region the hysteresis loop is linear. This motion proceeds as the external field is increased, until the wall has spanned the whole sample as in configuration (c). At this point the sample is all magnetized in the same direction, which however does not coincides in general with the direction of the external applied field. From now on, further increasing the field, the magnetization proceeds by coherent rotation of the spins (d), until the saturation magnetization is reached (e), where all the spins are all aligned parallel to the direction of the applied field.

being integrated over different values of the control parameters, are characterized by larger effective exponents. This might explain some experimental results which give unusually large values for the exponents in the power law distributions of sizes and durations of avalanches [49]. Evaluating the effects of non-stationary conditions on the statistical behavior of a system is an issue that arises in many driven non-equilibrium situations, and is analyzed in a more general context in [50].

A detailed analysis of the data from many Barkhausen experiments reported in literature is given by Durin and Zapperi in their review [5], where the reliability of each experiment considered is also discussed. 
(a)

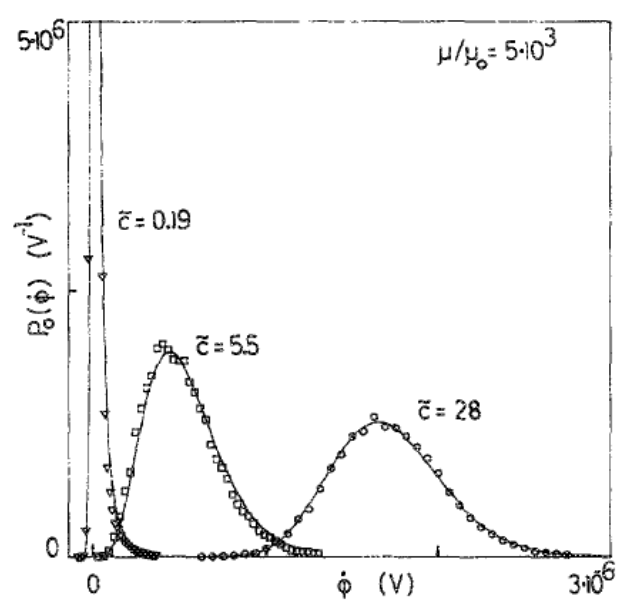

(b)

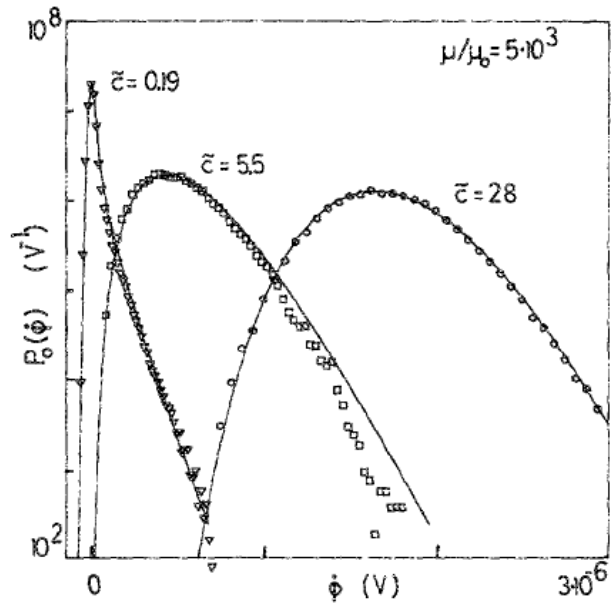

Figure 7. (a) Amplitude probability distribution $P_{0}(\dot{\Phi})$ (which corresponds to $P(V)$ in our notations) at three different values of the driving field rate. $\dot{\Phi}$ is the variation of magnetic flux assumed to be proportional to the wall velocity. $\tilde{c}$ is proportional to $c$. The experimental data on a polycrystalline $3 \% \mathrm{FeSi}$ sample are fitted with equation 5 (b) Same as (a), in semilogarithmic scale. (Reprinted with permission from [32]). [32] Copyright 1990 by the American Institute of Physics.

\subsection{Barkhausen noise phenomenology}

BN has some remarkable statistical properties, that are nowadays measured with great precision in experiments. We briefly report the main experimental results here.

2.4.1 Signal amplitude distribution. The amplitude $V$ of the BN signal, i. e. the instantaneous value of the voltage, is found to follow a distribution well fitted by

$$
P(V) \propto V^{-(1-c)} \exp \left(-V / V_{0}\right)
$$

where $c$ is proportional to the driving field rate, and $V_{0}$ is some characteristic cut-off value (see figure 7).

The amplitude distribution of the signal is an average quantity over avalanches. To go more into detail, and investigate the statistical properties of the single avalanches, it is necessary to define precisely where a jump starts and ends. This is commonly done by fixing a threshold value, which cannot be strictly zero due to the unavoidable presence of background noise (see figures 8. 9). That of signal thresholding is however a very complicated issue, which arises in many cases when one has to analyze temporal series, and that would 


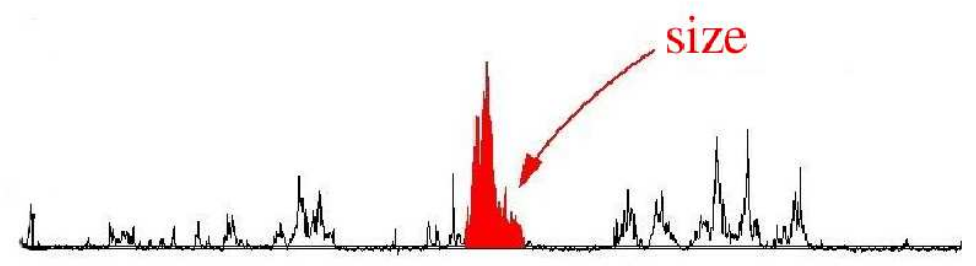

Figure 8. Size of an avalanche.

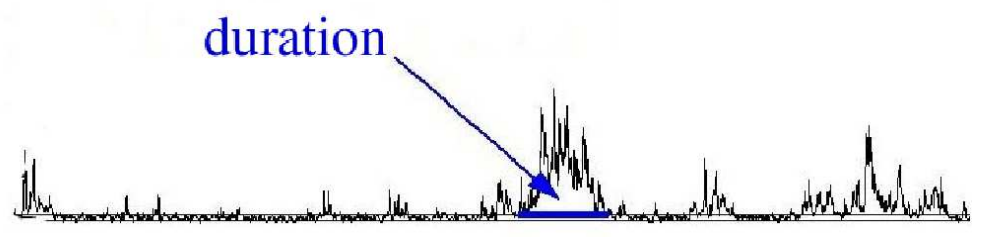

Figure 9. Duration of an avalanche.

need further attention. The choice of the threshold is crucial, since it defines the objects whose statistical properties one is looking at, and the absence of a clear rule makes the identification of single pulses rather ambiguous. On the other hand, signal thresholding is inescapable if one wants to analyze directly experimental time series, it would therefore be desirable to be able at least to control its effects on the measured quantities. An alternative approach to the statistical analysis of the signals, which bypasses completely ambiguous thresholding methods, is to focus on the spectral properties, which indicate temporal correlations in the system, and then try to infer from there the statistical properties of the avalanches. This is indeed possible in some cases thanks to the fact that the power spectra exponents are related to the exponents in the size and time distribution of pulses as it was shown by Kuntz and Sethna [51] for the random field Ising model and some of its variants, and by Laurson, Alava, and Zapperi [52] for the case of sand piles.

In the case of $\mathrm{BN}$, the experimental data have been shown to be relatively insensitive to the exact choice of the threshold [53]. Background noise limits the detection of small avalanches. Choosing the threshold below its typical amplitude would result in over-estimating very small avalanches, but it is expected not to affect sensibly the rest of their distribution. The characteristic signal amplitude $V_{0}$ in equation (5) gives a good reference value for the threshold, which is usually taken between 5 and $15 \%$ of $V_{0}$. 
2.4.2 Distribution of sizes and durations of Barkhausen pulses. Once the pulses are well defined one can look at the statistics of their sizes and durations. By drawing an histogram it appears that they are also both characterized by power law distributions, limited by an exponential cut-off: the probability of having a pulse of a given size has the form

$$
p(S)=S^{-\tau} F_{S}\left(S / S_{0}\right)
$$

where $F_{S}$ is some scaling function that accounts for the cut-off, and $S_{0}$ is some characteristic avalanche size.

The distribution of durations has a similar behavior: the probability of having a pulse of duration $T$ is given by

$$
p(T)=T^{-\alpha} F_{T}\left(T / T_{0}\right)
$$

where again $T_{0}$ is some characteristic time, and $F_{T}$ accounts for the exponential cut-off.

2.4.3 Universality classes. By repeating BN experiments on different samples of different materials and in different external conditions, it has been observed that the exponents characterizing the power law distributions are universal, and tend to cluster around two sets of values (see figure 10) [54]. In soft magnets, this allows to identify two distinct universality classes, which are named long range and short range, according to the kind of elastic interaction which dominates the behavior of the magnetic walls.

The two classes are characterized by exponents that, in the zero frequency limit of the applied field, take the values $\tau \simeq 1.50, \alpha \simeq 2.0$ for materials in the long range class, and $\tau \simeq 1.27, \alpha \simeq 1.5$ for those in the short range class, respectively for the avalanches' sizes and durations distributions (see figure 101).

Typical materials belonging to the long range class are polycrystalline $\mathrm{FeSi}$ with high $\mathrm{Si}$ content, and partially crystallized $\mathrm{Fe}_{64} \mathrm{Co}_{21} B_{15}$ amorphous alloys. Short range behavior is observed for instance on Perminvar $\left(\mathrm{Fe}_{30} \mathrm{Ni}_{45} \mathrm{Co}_{25}\right)$ and amorphous alloys with composition $\mathrm{Fe}_{x} \mathrm{Co}_{85-x} \mathrm{~B}_{15}$ under tensile stress.

We will restrict here to materials belonging to the long range class, since for these systems the mean field description given by the ABBM model, which is the focus of this review, turns out to be exact for universal properties at long length and time scales (see section 3.5 ). 



Figure 10. The data refer to Barkhausen measurements in different materials at the lowest available driving frequency. (a) Distribution of size of avalanches for different long range (filled symbols) and short range (empty symbols) materials. A solid line corresponding to slope 1.5 (long range) and a dashed line corresponding to slope 1.27 (short range) are shown for comparison. (b)

Distribution of duration of avalanches for different long range (filled symbols) and short range

(filled symbols) materials. A solid line corresponding to slope 2.0 (long range) and a dashed line corresponding to slope 1.5 (short range) are shown for comparison. (Reprinted with permission from [54]). [54] Copyright 2000 by the American Physical Society.

2.4.4 Distributions of pulses at finite driving rates. The values $\tau \simeq 1.5$ and $\alpha \simeq 2.0$ for the long range elasticity class, refer to experiments performed in the quasi-static limit, namely, such that the applied field is increased very slowly with respect to the typical time scale of the system, in such a way that the system has time to rearrange after every infinitesimal change of the field before the field is increased again. 


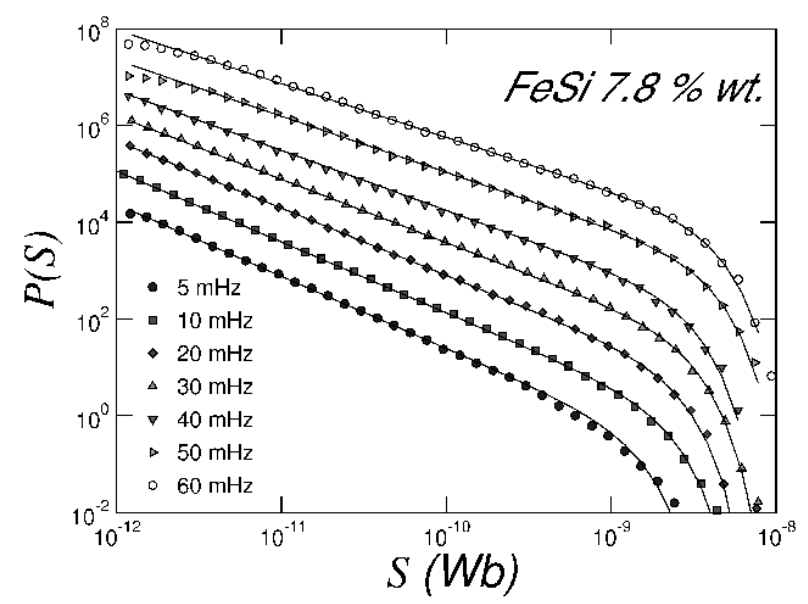

Figure 11. Cumulated distribution of sizes $P(S)=\int_{S}^{\infty} p(s) d s$ for a polycrystalline FeSi material at different applied field frequencies. The solid lines are fits with equation 6 and with

$\tau-1=(1-c) / 2$. The exponent $\tau$ varies linearly with frequency between 1.5 and 1.15 . (Reprinted with permission from [5]). [5] Copyright 2005 by Academic Press.

In experiments where the frequency of the field is finite but still small, one can observe a linear dependence of the exponents $\alpha$ and $\tau$ on the driving field rate $c$, well fitted by the expressions $\alpha=2-c$, and $\tau=(3-c) / 2$ (see figure 11, where the cumulated distribution of sizes of BN pulse for a polycrystalline $F e S i$ sample is shown for seven values of the field sweep rate). No similar dependence has been observed in materials belonging to the short range class, where the exponents keep their quasistatic limit values, independently of the driving field rate.

\subsubsection{Threshold in the field rate, transition from intermittent to contin-} uous regime. The variation of the exponents $\alpha$ and $\tau$ with the driving field rate $c$ reflects the fact that, increasing the sweep rate, large events become more frequent. Indeed, even without a statistical analysis, it is clear by eye inspection that the Barkhausen signals become more and more pronounced as the frequency of the applied field is increased (see figure 12).

Further increasing the driving field rate, it is observed that, above some well defined threshold, the typical intermittent behavior is lost, single pulses disappear, and the magnetization reverses in a unique avalanche. This is a sort of meta-phase transition, which separates two completely different regimes of the dynamics. It would be interesting to see how the system approaches the critical driving rate, by monitoring for example the quiescence times separating the avalanches as $c$ approaches the threshold value. This, to our knowledge, has never been done. A similar situation arises for example in sheared granular matter, where a threshold driving separates the stick-slip form the sliding 


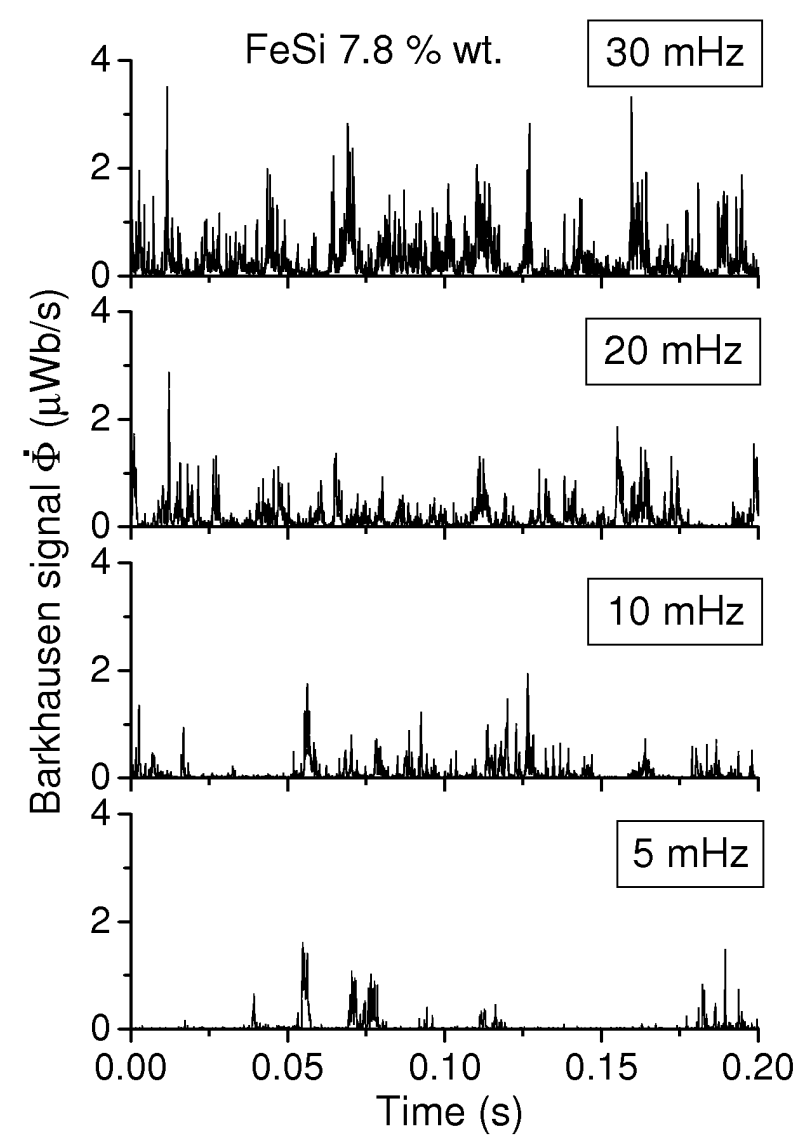

Figure 12. Barkhausen signal recorded at increasing sweep rates. (Reprinted with permission from [5]). [5] Copyright 2005 by Academic Press.

regime. This effect is due to avalanches overlapping aver time: since at finite driving rates the external field increases during the avalanche, it can keep active an avalanche that would have stopped otherwise (see subsection 4.4 and the paper by White and Dahmen [55]).

2.4.6 Cut-off in the distribution of sizes and durations. The power law distributions of sizes and durations of avalanches are limited by an exponential cut-off, which turns out to be related to the demagnetizing field (see subsection 4.6), which in its turn depends on the sample geometry. Experiments repeated on samples with different geometries, indeed confirm that the position of the exponential cut-offs $S_{0}$ and $T_{0}$ in the distributions (6) varies with the shape of the sample. However, a precise experimental evaluation of the cut-off dependence on the parameter $k$ measuring the strength of the demagnetizing field 


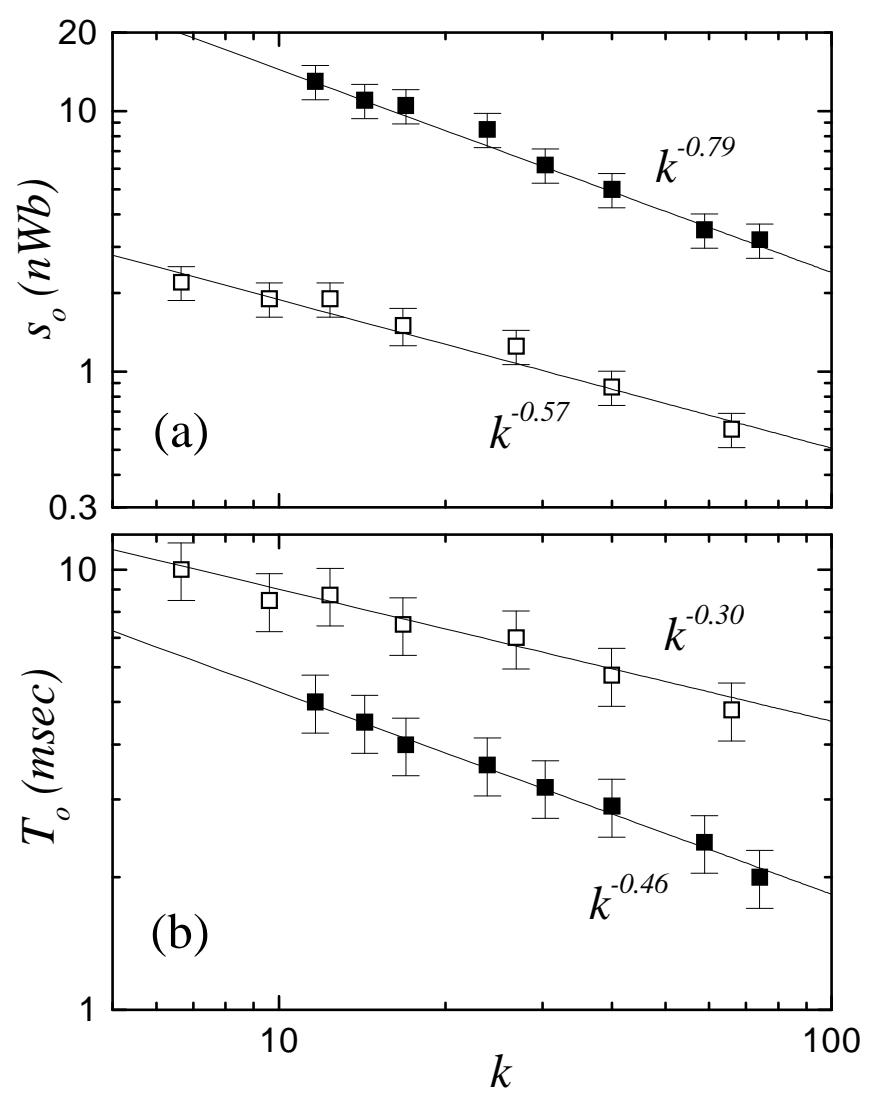

Figure 13. Top graph: experimental estimations of the cut-off $S_{0}$ of Barkhausen pulse size distribution as a function of the demagnetizing factor $k$ in a FeSi $6.5 \mathrm{wt} \%$ alloy (empty symbols), and an amorphous alloy $\mathrm{Fe}_{21} \mathrm{Co}_{64} B_{15}$ under stress (filled symbols). The empty symbols correspond to the long range class considered here. Bottom graph: same as the top for the cut-off

$T_{0}$ of the Barkhausen pulse duration distribution. (Reprinted with permission from [54]). [54] Copyright 2000 by the American Physical Society.

is very difficult, also due to the further dependence of the distribution on the driving rate.

Earlier studies [56] report the scaling $S_{0} \sim k^{-1}$, and $T_{0} \sim k^{-1 / 2}$. The most reliable experiments are probably those reported in [54,57], done by taking measurements on the same ribbon repeatedly cut along one direction, in order to modify the effect of the demagnetizing field, while stresses and internal disorder are kept constant. For a FeSi sample, the scalings reported are $S_{0} \sim$ $k^{-0.57}$, and $T_{0} \sim k^{-0.30}$ (see figure [13).

2.4.7 Average pulse shape. Averaging avalanches of the same duration $T$, one obtains, for every given $T$ a characteristic pulse shape. 


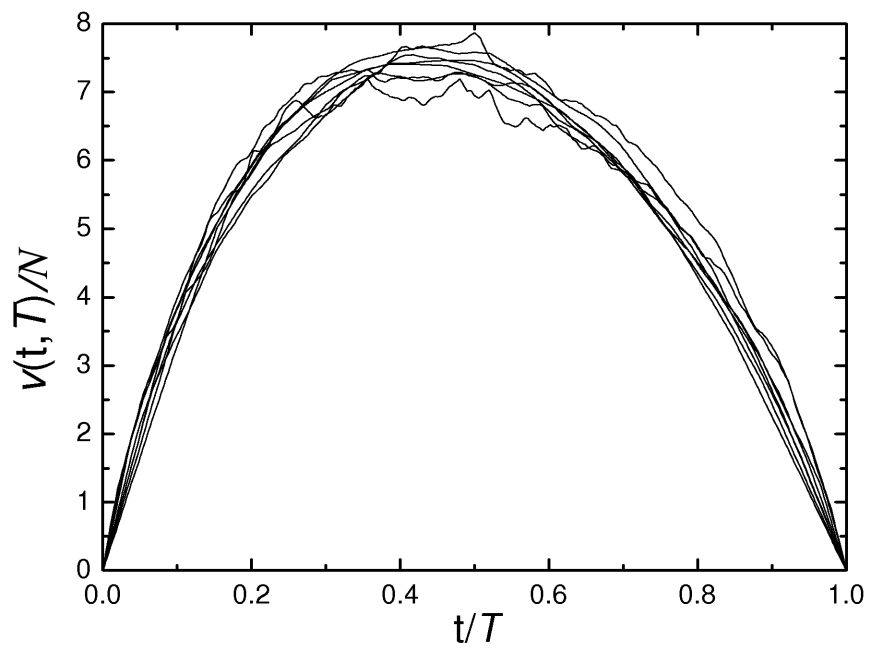

Figure 14. Attempt to collapse average pulses of different durations onto the same function. The data are from a BN experiment on a polycrystalline $F e S i$ sample. Pulses are normalized with $N \propto \int_{0}^{1} d(t / T)\langle v(t \mid T)\rangle$. The curves are averages over several pulses for each of eight different values of $T$, ranging from 0,16 to 2, $03 \mathrm{~ms}$. (Reprinted with permission from [5]). [5] Copyright 2005 by Academic Press.

$$
\langle V(t \mid T)\rangle
$$

In analogy to conventional critical phenomena one might expect that average pulses of different durations, properly rescaled by some power of their duration, will depend on $t$ only through the scaling variable $t / T$, and therefore collapse onto the same universal function. This quantity was recently proposed in [1] to test theory against experiments, and indeed it provides a much stringent tool than the simple comparison of scalar quantities, such as critical exponents.

As shown in figure 14, which reports data from a BN experiment on a polycrystalline $\mathrm{FeSi}$ sample, the rescaling of experimental pulses can approximately be performed. As we will see, the shape of the scaling function is influenced by some non-universal effects that however disappear, as expected, on very long time scales.

2.4.8 Power spectra. In most of earlier literature, experimental papers focus on the detection of the power spectra of the noise. The most accurate report of power spectra are reported in the seminal paper by Alessandro et al. [32] in experiments on a $3 \% \mathrm{FeSi}$ sample. For experiments on single crystals and other polycrystalline $\mathrm{FeSi}$ materials see also [58].

For materials in the long range universality class the power spectra is found 


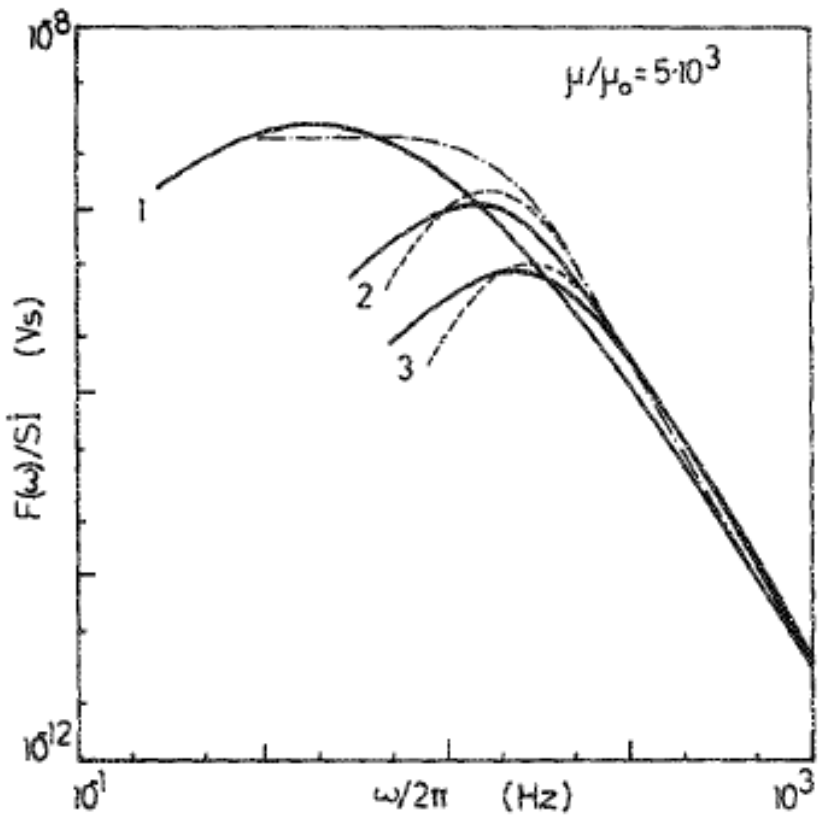

Figure 15. Log-log plot of the power spectra divided by the driving field rate, versus frequency measured in a polycrystalline $3 \% \mathrm{FeSi}$ sample. The three solid lines are the experimental results for three different driving field rates, the dotted lines are predictions from the ABBM model. (Reprinted with permission from [32]). [32] Copyright 1990 by the American Institute of Physics.

to decay at large frequencies $\omega$ as $\omega^{-2}$ (see figure 15), although in same cases a deviation from this scaling is observed at intermediate frequencies. At small frequencies the spectrum is characterized by the presence of a maximum. The position of the peak depends on the driving field rate $c$ : it occurs at a frequency $\omega_{M}$ roughly proportional to $c^{1 / 2}$, and with an amplitude $F_{M}$ that scales as $c$ in the intermittent regime (slow driving), while it is roughly constant in the continuous regime (fast driving) (see figure 16 where $F_{M} / c$ is plotted versus $c$ ).

The behavior of the power spectra reported in [32] and shown in figure 15 is indeed common to many ferromagnetic materials. Similar spectra are found for example in $N i F e$ alloys with vanishing anisotropies [59], grain oriented FeSi alloys [47], FeSi single crystals [60].

At frequencies below the peak the power spectrum has been claimed to behave like some power $\omega^{\psi}$, with approximately $\psi \simeq 0.6$. However, these claims are based on fits of data that are spread on less than one decade, and therefore are not conclusive.

Although the large $\omega$ tail and the existence of the peak are reproduced by the theory, the correct interpretation of all the details in the complex behavior of the frequency spectrum still represents a challenging problem. In principle the 
(a)

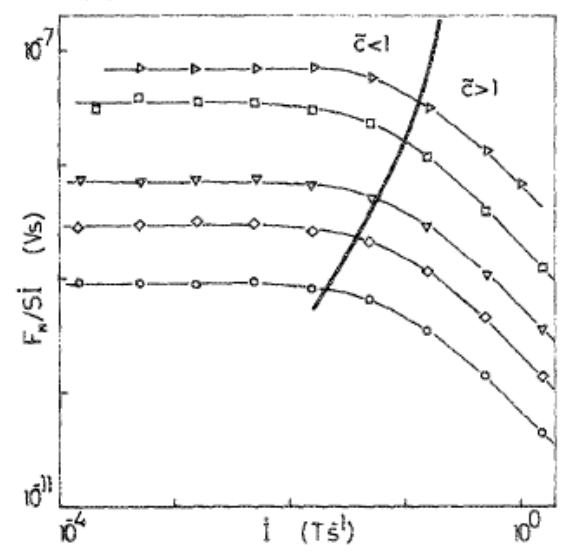

(b)

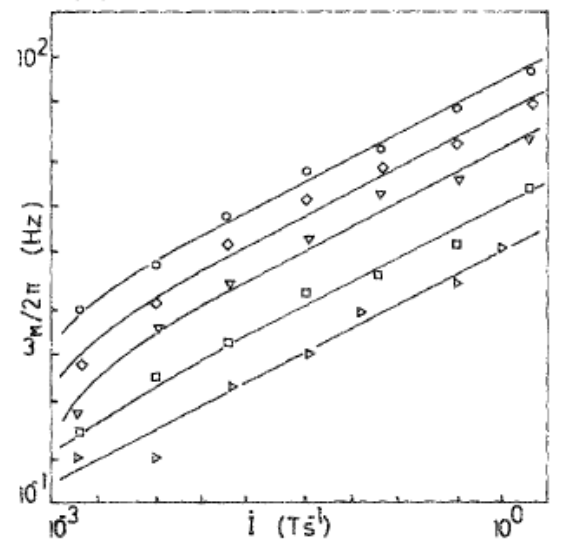

Figure 16. (a) Amplitude of the maximum $F_{M}$ in the frequency spectrum divided by driving field rate versus driving field rate in log-log scale. Different data-sets refer to different values of the permeability. The bold line separates the two regimes intermittent and continuous. $\tilde{c}$ is equivalent to $c$ in our notations. (b) Same as (a), for the frequency $\omega_{M}$ corresponding to the maximum of the power spectrum. (Reprinted with permission from [32]). [32] Copyright 1990 by the American Institute of Physics.

contributions from the correlations among different avalanches, and that from the correlations within avalanches are mixed together in the power spectrum, which therefore is not easily amenable of physical interpretation.

Some authors have also considered higher order spectra to further characterize the BN signal $[61,62]$. In principle this analysis could indicate whether events on a certain scale would systematically precede events on another scale. While no precursor events have been found, time asymmetries of the signal emerge from the analysis of the imaginary part of the moment of order 1.5 consistently with that observed in the skewed pulse shape (see below).

2.4.9 Summary of experimental observations. It is useful at this point to summarize the main phenomenological observations collected from different $\mathrm{BN}$ experiments, that we will want to compare to the theoretical predictions in the next sections.

For soft materials with long range interactions:

(i) The distribution of the intensity of the BN signal follows a power law decay with an exponent $1-c$ where $c$ is proportional to the driving field rate.

(ii) The sizes and durations of avalanches are power law distributed. In experiments performed in the quasi-static limit the exponents are $\alpha \simeq 1.5$ and $\tau \simeq 2.0$, respectively.

(iii) The distributions of sizes and durations of avalanches flatten as the sweep 
rate is increased. The corresponding exponents vary linearly with the field rate $c$, and their values are well fitted by the relations $\alpha=2-c$, and $\tau=(3-c) / 2$.

(iv) The previous statement hold as long as the sweep rate is not too high: there exist a well defined threshold in the driving field rate $c$ above which the Barkhausen pulses become indistinguishable and magnetization reverses in a unique avalanche.

(v) The exponential cut-offs $S_{0}$, and $T_{0}$ in the distributions of sizes and durations of avalanches are related to the sample geometry through the demagnetizing field, and scale as powers of $k$.

(vi) Average pulses properly rescaled by their duration can approximately be collapsed onto the same function.

(vii) The power spectrum decays as $\omega^{-2}$ at large frequencies. At small frequencies is characterized by a peak that occurs at $\omega_{M} \simeq c^{1 / 2}$, with an amplitude that in the slow driving regime scales roughly as $F_{M} \simeq c$.

\subsection{Modern theoretical approaches to Barkhausen noise}

Recent theoretical studies on BN have moved from a pure phenomenological description, where $\mathrm{BN}$ is considered as a superposition of elementary events, without any connection to the microscopic dynamic, to more physical and detailed descriptions which take into account the relevant interactions governing the dynamics of the magnetization reversal process.

The theory has then developed on two different but related avenues: one follows a microscopic description and models the magnetic system through interacting spins. In this framework, the criticality observed in BN experiment is explained in terms of the proximity of a disorder driven non-equilibrium phase transition, which is observed in the zero temperature random field Ising model (RFIM). The other approach describes the magnetization process in mesoscopic terms, through the dynamics of the domain walls. In this case, the criticality is related to the depinning transition occurring when the applied field overcomes a critical threshold value. We will briefly discuss here the first approach, and refer to the next sections for the second one, which is the one taken in this is review.

The attempt to model the Barkhausen phenomenon in terms of spin models is indeed appealing. Thanks to universality on expects the statistical properties of $\mathrm{BN}$ to be independent of the microscopic details of the system, provided that the relevant symmetries of the problem are properly taken into account. This allows in principle to derive macroscopic properties without going through the analysis of complicated micromagnetic equations, which are very difficult to treat analytically. Instead, one models the avalanche dynamics with simplified rules for the evolution of integer valued spins. This approach allows both to 
perform large scale simulations, and to derive some theoretical result.

In particular, among all the spin models, the non-equilibrium random field Ising model (RFIM), proposed by Sethna et al. [63] as a model for avalanche dynamics in BN, has been widely studied both analytically and numerically [1,64-69]. In this model integer valued spins $s_{i}= \pm 1$ are assigned to each site of a $d$-dimensional lattice, and interact with the nearest neighbors through a ferromagnetic coupling $J$. The spins are coupled to the external field $H$, and to a quenched random field $h_{i}$ extracted from a Gaussian distribution with variance $R$, which is associated to each site of the lattice, and is supposed to mimic the presence of disorder. The Hamiltonian reads

$$
\mathcal{H}=-\sum_{\langle i, j\rangle} J s_{i} s_{j}-\sum_{i}\left(H(t)+h_{i}\right) s_{i}
$$

where the first sum is restricted to the nearest neighbors. In the nonequilibrium zero temperature dynamics proposed by Sethna and co-workers [63], at each time step the spins align with the local field:

$$
s_{i}=\operatorname{sign}\left(J \sum_{j} s_{j}+H(t)+h_{i}\right),
$$

where, again, the first sum is restricted to the nearest neighbors of the spin $i$. Starting from a configuration with all the spins down, the field $H$ is slowly ramped from $-\infty$ to $+\infty$. A single spin flip can cause nearest neighboring spins to flip, and trigger an avalanche. In the quasistatic limit the field $H(t)$ is kept constant during the avalanches.

For small values of $R$, the random field is not strong enough to stop the avalanches, and the hysteresis loop has a squared shape, with a large jump at a critical field $H_{c}$ at which the big avalanche starts. When $R$ is large, only small avalanches are observed: essentially every spin flips independently when $H(t)$ is large enough to overcome the local pinning field $h_{i}$, plus the comparably small energetic contribution from the coupling $J$ with the neighboring spins. The two regimes are separated by a critical value $R_{c}$ of the variance of the random field, for which the avalanches are power-law distributed.

This model predicts that for the critical value of the disorder $R=R_{c}$, at the critical point, in $d=3$, and in the quasi-static limit, the exponents in the power-law distributions of size and duration of the avalanches are given by $\tau \simeq 1.6$, and $\alpha \simeq 2.05$. These values have been obtained both by large scale numerical simulations [67], and with an epsilon expansion around the mean field solution, that has been be worked out analytically [66].

The main problem with this approach is that RFIM predicts scaling only 


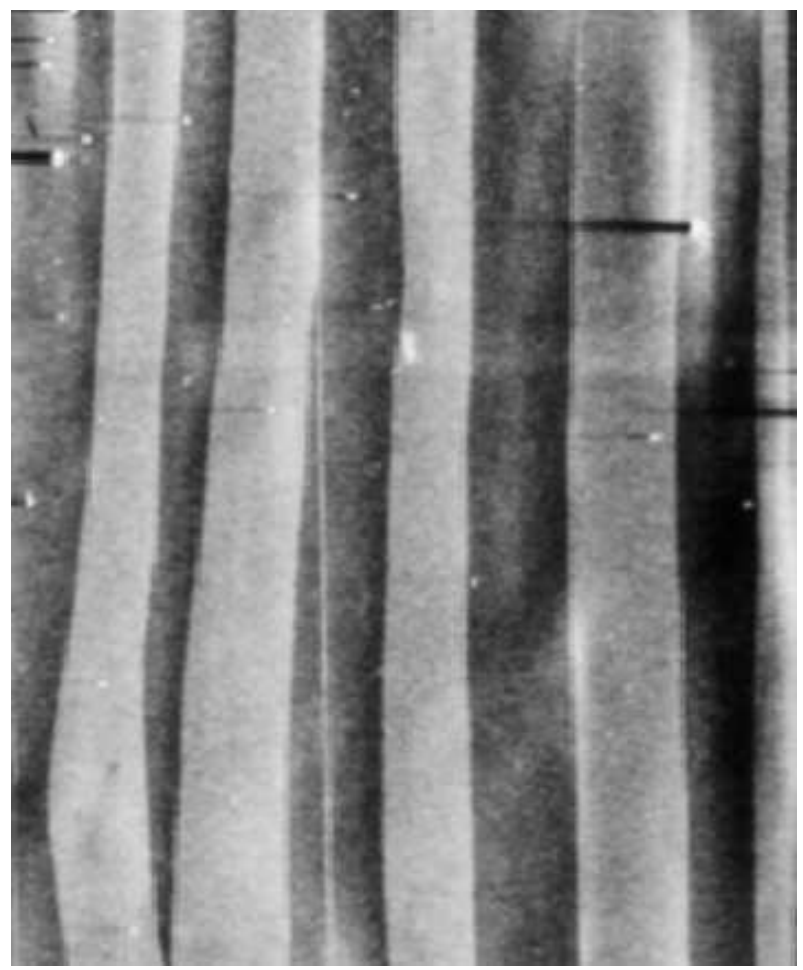

Figure 17. Domain structure of $\mathrm{Fe}_{21} \mathrm{Co}_{64} B_{15}$ amorphous alloy observed by scanning electron microscopy. This is the typical structure observed in soft ferromagnetic materials. (Reprinted with permission from [56]). [56] Copyright 1998 by the American Physical Society.

at a critical value of the disorder, while there is not reason to expect this condition to be satisfied in experiments. Another problem is that the results for the critical exponents in the non-equilibrium RFIM are only marginally compatible with the values observed in experiments, where the exponents are around $\tau \simeq 1.50, \alpha \simeq 2.0$, or $\tau \simeq 1.27, \alpha \simeq 1.5$ depending on the range of dipolar interactions.

More importantly, even a rough investigation of the qualitative behavior of the non-equilibrium RFIM shows that the domain structure is very different from the one observed in real magnets (see figure 17). While in the RFIM avalanches nucleate in different points of the system and grow with a fractal spatial structure, in real soft magnets domain walls are rather flat [2].

In the RFIM the magnetization proceeds simultaneously by two different mechanisms, nucleation and wall displacement, while in real soft magnets (and in the central part of the loop, where $\mathrm{BN}$ is measured) the wall movement is the only relevant mechanism of magnetization reversal: the dynamic evolves by the motion of existing boundaries, rather then by nucleating new domains. As a consequence, the RFIM, as it is, is inadequate as a model for BN in soft mag- 
nets, while it could be an interesting starting point to describe magnetization dynamics in hard magnetic materials.

The non-equilibrium RFIM neglects dipolar interactions, which originates, both from free dipoles which are present on the sample surface, and from those on the magnetic interface. Magnetostatic dipolar interactions due to the presence of "free magnetic charges" on the sample boundaries, generate a long range demagnetizing field, which opposes the external applied field. The most relevant ingredient missing in the RFIM is indeed the demagnetizing field, which reflects in the flat domain domain structure observed in soft materials (see figure 17), very different from the structure found in the RFIM, where demagnetizing forces are not taken into account. The other crucial effect of the demagnetizing field, as we discuss below, is that it prevents the formation of large avalanches.

Several variants of the RFIM have been proposed to overcome these problems. A particularly successful one, the front propagation model (FPM), originally introduced in [70], has been extensively analyzed in [51,69,71] as a model for BN. In this variant of the RFIM, the dynamics is such that only spins close to an existing domin boundary take part to the dynamics, while spins not adjacent to an avalanche front are prohibited to flip, even when it would be energetically favorable, so that the nucleation of new domains is totally suppressed. This can also be implemented by introducing in the RFIM Hamiltonian a long range term mimicking the demagnetizing field. The FPM gives exponents $\tau \simeq 1.27$, and $\alpha \simeq 1.72$ in $d=3$, which are compatible with $\mathrm{BN}$ experiments for soft magnets in the short range universality class.

It has to be stressed that, unlike the RFIM, the FPM exhibits critical behavior in the whole low disorder regime $R<R_{c}$ and not just at the critical value $R_{c}$. Indeed, the demagnetizing field opposes the reversal process and forbids the magnetization to change abruptly, as it is the case, below $R_{c}$, in the original non-equilibrium RFIM: the demagnetizing field reduces the effective field until it stops the domain growth, which will start again with a new avalanche only by the increase of the external field. This mechanism will be treated extensively in the next section. The criticality observed is therefore not related to the disorder induced transition of the non-equilibrium RFIM, but to a depinning transition.

Dipolar interactions between free dipoles on the magnetic wall produce an effective elasticity between different parts of the domain wall, that can be short range or long range, depending on the material. The long range elastic interaction has been claimed to lower the upper critical dimension to $\mathrm{d}=3$ [56]. In such materials, a mean field approximation is therefore expected to apply. In mean field, the front propagation model gives $\tau \simeq 1.5$, in good agreement with experiment on materials in the long range universality class.

Another point that should be considered carefully in any RFIM approach 
to $\mathrm{BN}$ regards the kind of disorder it represents, since random fields are not present in real magnets. It is therefore crucial to understand whether the RFIM non-equilibrium phase transition represents a broad enough universality class that is preserved when other kinds of disorder are introduced. This question is still controversial. Several studies have attempted to understand by numerical simulations whether other disordered spin models belong to the same universality class as the RFIM. Examples include the non-equilibrium random bond Ising model (RBIM), with Hamiltonian

$$
\mathcal{H}=-\sum_{\langle i, j\rangle} J_{i, j} s_{i} s_{j}-\sum_{i} H(t) s_{i}
$$

where the disorder enters into the exchange couplings $J_{i, j}$, and the nonequilibrium site diluted Ising model (SDIM), described by

$$
\mathcal{H}=-\sum_{i, j} J c_{i} c_{j} s_{i} s_{j}-\sum_{i} H(t) c_{i} s_{i}
$$

where the quenched variables $c_{i}$, taking the values $[0,1]$, indicate whether a given site represents a magnetic spin or not, thus modeling the presence of non-magnetic inclusions in the material.

Simulations show that both the non-equilibrium RBIM [72] and the nonequilibrium SDIM [73] exhibit a disorder induced phase transition, with a behavior qualitatively similar to that arising in the RFIM. The critical exponents for the avalanche dynamics differ slightly, but are compatible, with those of the RFIM, suggesting that RFIM may indeed represent a broad universality class. The robustness of RFIM with respect to different kinds of disorder is also discussed by Dahmen and Sethna in [66], where it is argued that universality is expected to hold on the base of renormalization group calculations. The authors suggest that although RBIM and the random anisotropy model have higher symmetries then the RFIM in equilibrium, they have the same symmetries in non-equilibrium conditions, since the external field at the critical point breaks the up-down symmetry.

To conclude our short discussion of spin models, we can summarize that the simple RFIM is inadequate to describe the Barkhausen effect in soft magnets, rather it could be a good starting point to model magnetization reversal process in hard magnets. The FPM, obtained from the RFIM by incorporating the demagnetizing field, and suppressing domains nucleation, is instead a more adequate spin model for soft magnets, and give results in good agreement with experiments on materials in the short range universality class for $d=3$, and for the long range in mean field.

It is however very important to stress that in the FPM, the observed critical 
behavior is not related to the proximity of the RFIM non-equilibrium critical point (indeed criticality is observed for all $R<R_{c}$, i.e. in the whole low disorder regime), but rather to the depinning transition of the magnetic interface that will be discussed extensively in the next sections.

In some sense spin models can be more general then interface models, as can easily deal for example with interface overhangs, however, in the case of soft magnets, given the nature of the transition, a simplified approach in terms of domain wall dynamics, seems more convenient, and has the advantage to allow to treat separately domain wall propagation and nucleation. The FPM naturally provides a discretized way to implement the front dynamics, very suitable for simulations. An alternative approach, which we follow in this review, is to formulate directly an equation of motion for the propagating wall.

\section{The ABBM model}

The first important theoretical approach to BN was done in 1990 by Alessandro, Beatrice, Bertotti, and Montorsi, who proposed a phenomenological model, which was then named after the authors of the two companion papers [31,32], one dealing with theory, the other with experiments. By-passing all the difficulties that appear when trying to approach the problem from basic micromagnetic equations, Alessandro et al. managed to capture the essential ingredients at the origin of $\mathrm{BN}$, by formulating a very simplified equation for the domain wall dynamics. Their model turned then out to be extremely successful in reproducing the experimental observations, and played a fundamental role in most of the successive theoretical progress, and in the development of a consistent theory for BN.

The ABBM model was inspired by the work of Neél $[74,75]$, who was the first to introduce a random energy model in the study of hysteresis: in Neél's model, the complex domain structure usually present in a magnetized sample is replaced by a single domain wall, moving in a random energy landscape, which is supposed to account for all the interactions among walls, and of the walls with the medium. The energy profile is constructed with a sequence of equispaced parabolic arcs, whose curvatures are extracted from a Gaussian distribution, in such a way that, on small scales, the motion is reversible, while, on large scales, the wall moves in random irreversible jumps. This model was introduced to explain in simple terms the Rayleigh law in hysteresis.

In the Alessandro et al. approach, the idea of a random energy landscape is generalized, and used to construct a stochastic equation for the domain wall dynamics, in order to describe the Barkhausen effect. The equation is derived by following a previous work by Williams et al. [76], who studied the dynamics of a single domain wall under an external field in a FeSi single 


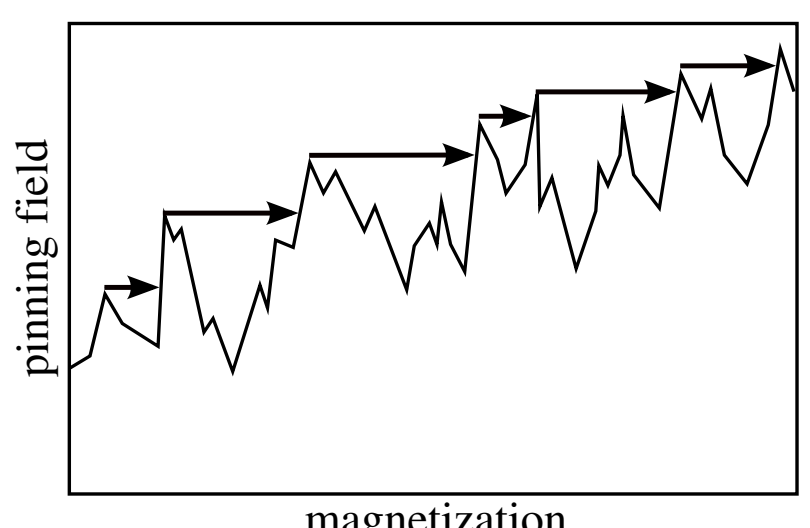

Figure 18. Random energy landscape representing the pinning field, the arrows represent the Barkhausen jumps. In random energy models, when the system has enough energy to overcome a barrier, it jumps irreversibly to the next valley, with a corresponding random jump in the magnetization. Then the system is stuck until the effective field has grown enough to overcome the new energy barrier.

crystal sample. In this work, the motion of the wall, limited by eddy currents damping, was described by a linear relation between the average velocity and the net field acting on the interface, given by the difference between the applied field $H(t)$ and some threshold field $H_{0}$, which includes all kinds of random (like interaction with pinning centers) and non-random (like dipolar forces and demagnetizing fields) interactions (see figure 18).

The ABBM model is derived by making a more stringent claim, namely by assuming that the linear relation between velocity and effective fields holds not only on average, but also on the instantaneous velocity. By separating in $H_{0}$ the random contribution of the pinning field $H_{p}$ from the demagnetizing component $H_{d}$, one could then write

$$
v(t) \propto H(t)-\left(H_{d}+H_{p}\right)
$$

The further assumption is that the pinning field is Brownian correlated (see Eq. 15 below), as suggested by some experimental evidence in special system containing just one active wall $[77,78]$. Let us point out that this assumption is quite crucial, since an uncorrelated pinning field, as the one considered in the original work by Neél, would not give rise to power law distributed magnetization events.

In the following section we will first describe the ABBM equation, as it was formulated in $[31,32]$. Then, in order to put this model in a more general framework, we turn to the description of the dynamics of a magnetic elastic interface in a random media. Following [79], we describe a depinning interface model for $\mathrm{BN}$, that in the mean field approximation gives back the ABBM 
model [56]. As we will see, this will also clarify the origin of the correlated effective pinning field.

\section{1 $A B B M$ as a phenomenological model}

The ABBM theory assumes that there is just one single rigid wall separating two domains of opposite magnetization. The system has therefore just one degree of freedom, and the dynamics is described in terms of a one-dimensional equation of motion for the center of mass of the wall. The wall moves under the action of an applied driving field in an effective Brownian correlated pinning field, mimicking the presence of disorder in the sample, as discussed above.

Without loss of generality, we choose the origin of the coordinate indicating the position of the wall at the center of the sample, i.e. at the zero magnetization domain configuration, so that, in what follows, the magnetization is proportional to the position of the wall. The equation of motion for the center of mass of the wall is then an equation for the magnetization.

The motion is assumed to be overdamped, so that the velocity of the wall, which is proportional to the derivative of the magnetization, is equal to the sum of the forces acting on the interface, with a damping coefficient $\Gamma$, characterizing the amount of dissipation:

$$
\Gamma \frac{\partial m}{\partial t}=H(t)-k m+W(m)
$$

As mentioned before, the total force acting on the wall, on the r.h.s. of the equation, includes two terms, besides the time-dependent applied magnetic field $H(t)$, as in equation (13). One is the demagnetizing field $H_{d}$ generated by free magnetic charges on the boundary of the sample, which opposes the external field and here is assumed to be proportional to the magnetization $H_{d}=-k m$, through a constant $k$ (demagnetizing factor) that takes into account the geometry of the sample. The other one is the random pinning field $H_{p}=W(m)$, which accounts for defects and non-magnetic impurities present in the sample, and it is assumed to be Brownian correlated in the magnetization:

$$
\left\langle\left|W(m)-W\left(m^{\prime}\right)\right|^{2}\right\rangle=2 D\left|m-m^{\prime}\right|
$$

\subsection{Limitations of the ABBM model}

The ABBM model has the advantage to be exactly solvable, and to reproduce with striking accuracy most of the statistical properties observed in the experiments for soft magnetic materials in the long range universality class, 
described in section 2.4. However, it is worth to underline its main limitations. One, that we will deal with in the last part of the review (see section 5), is that it is unable to reproduce some features of non-universal origin, in particular, the asymmetric shape of Barkhausen pulses.

Another important limitation of the ABBM model is that it deals with a single domain wall, while in common experiments more interacting domains are present in the specimen and participate in the magnetization process. The ABBM predictions on the signal power spectra are for example cleanly observed in experiment where a single domain wall is present [78], while other experiments suggest that the power spectra changes when more domains are present $[80,81]$. To fully understand these experiments a model which includes many interacting domain walls would be needed. This point was indeed raised in the original paper [31], where the authors argue that in the case of many interacting domains, the same equation of motion approximately holds, once the correlation associated to the pinning field (see the discussion in section 4.8) is multiplied by the number of active walls.

Another feature of $\mathrm{BN}$ for which the description in terms of the $\mathrm{ABBM}$ model is unsatisfactory is the partial signal reproducibility observed in some experiments $[82,83]$, that could be due to small variations in the initial conditions or in the driving field rate or in the peak field, or to thermal effects. A model able to describe this behavior should treat many interacting degrees of freedom explicitly, and not in an effective manner as in the ABBM model.

A comparison point to point between ABBM model predictions and the experimental evidences, which are summarized in subsection 2.4.9, is postponed to subsection 4.9 .

\subsection{Elastic interfaces in a random media}

An analysis of the Barkhausen phenomenon at a mesoscopic level is done by describing the system as an elastic interface in a random media driven by an external field, and relates the criticality observed in the experiments to the well known depinning transition of the interface.

The response of an elastic manifold in a random media to an external driving force is a well studied problem in statistical physics, that arises in many contexts, for example in the study of fluids in porous media [84-88], flux lines in type II superconductors [89], charge density waves [90-92], and crack fronts in solids $[25,27,28,93-95]$. In the following we briefly review the main facts, for the case of a $d-1$ interface in $d$ dimensions, and refer to the extended literature for more details $[96,97]$.

Assuming that the motion is overdamped, so that inertial effects may be neglected, the general equation describing the dynamics of an elastic interface in a quenched random media, is obtained by equating the damping term to 
the total force acting on the interface:

$$
\Gamma \frac{\partial z}{\partial t}(\vec{r}, t)=-\frac{\delta E}{\delta z}
$$

where, assuming that there are no overhangs, $z$ defines the position of the interface as a function of the $d-1$ dimensional coordinate $\vec{r}$ on the substrate plane, and of time $t$. The coefficient $\Gamma$ is the damping coefficient, ruling the rate of dissipation.

The simplest equation of motion for an interface in an isotropic medium is the quenched Edward Wilkinson equation in a driving field, given by

$$
\Gamma \frac{\partial z}{\partial t}(\vec{r}, t)=\vec{F}(t)+\nu \nabla^{2} z(\vec{r}, t)+\eta(z(\vec{r}, t), \vec{r}),
$$

where $\vec{F}$ is a uniform, eventually time dependent, applied force, $\nabla^{2} z$ is the elastic term, whose coefficient $\nu$ is the surface tension, and $\eta(z, \vec{r})$ is the noise term, that mimics the presence of quenched disorder in the system. We consider here the zero temperature limit, where thermal noise is negligible with respect to quenched noise. A more general equation should include a kinematically generated nonlinearity of the Kardar-Parisi-Zhang type, which changes the asymptotic scaling. However this term vanishes at the critical point and therefore Eq. 17] is expected to have the correct critical behavior at the depinning transition (see below).

The quenched disorder is described by a random potential $V(z, \vec{r})$, whose derivatives gives the local pinning field $\eta(z, \vec{r})$ in equation (17). In $d=3$, if $P_{i}=\left(x_{i}, y_{i}, z_{i}\right)$ are the coordinates of the point-like defects, and $\vec{r}_{i}=\left(x_{i}, y_{i}\right)$, then the random force is

$$
\eta(z, \vec{r})=-R \sum_{i} \delta\left(z-z_{i}\right) \delta^{2}\left(\vec{r}-\vec{r}_{i}\right)
$$

where the sum runs over the pinning points. Here we assume the random noise to be uncorrelated and Gaussian distributed, so that

$$
\left\langle\eta(z, \vec{r}) \eta\left(z^{\prime}, \vec{r}^{\prime}\right)\right\rangle=2 \delta\left(z-z^{\prime}\right) \delta^{2}\left(\vec{r}-\vec{r}^{\prime}\right)
$$

Systems described by equation (17) exhibit a second order dynamic phase transition, called the depinning transition. The order parameter is the velocity of the interface, and the control parameter is the applied force, that we assume uniform and perpendicular to the substrate plane.

The behavior of the system is sketched in figure 19, At small applied forces the interface is locked by the pinning potential and $v=0$. At some threshold 




Figure 19. Sketch of the behavior of the interface velocity as an order parameter for the second order non-equilibrium depinning transition.

value $F_{c}$ the force overcomes the pinning. For $F>F_{c}$ the interface moves with constant velocity. Just above the threshold value $F_{c}$ the interface moves in power law distributed jumps.

\subsection{Dynamics of a magnetic elastic interface}

In the case of a magnetic wall, the interface separates two domains with opposite magnetization. When an external field $\vec{H}$ is applied, the system tends to increase the domain with the largest component of the magnetization in the direction of the field, by domain wall displacement. Thus the field $\vec{H}$ acts as an effective force perpendicular to the wall, as shown schematically in figure 20, and we can replace $\vec{F}$ with $\vec{H}$ in equation (17).

Unlike many other cases where an elastic interface is driven in a disordered media, magnetic systems do not generally experience a depinning transition of the kind predicted by equation (17) and described in the previous section. This is due to the presence of other important interactions peculiar to the magnetic case, that modify the behavior of the interface in an essential way. 


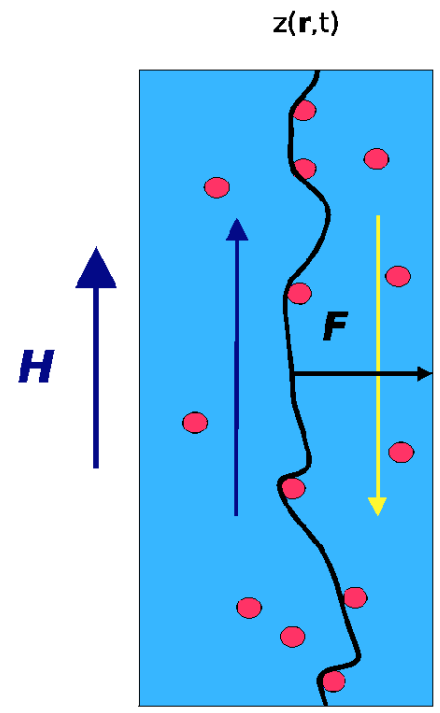

Figure 20. Magnetic wall in an external field $\vec{H}$. The system tends to increase the size of the domain magnetized in the direction of the external field, which therefore acts as an effective force perpendicular to the interface.

A first important step to adapt the general theory of elastic interfaces in random media in order to take into account the magnetic nature of the interactions was done by Urbach, Madison, and Markert [98] who modified equation (17) to include the effect of the demagnetizing field (see also [99]). The demagnetizing field is the field generated by the presence of free magnetic charges on the boundaries of the sample (see figure 21(a)). It opposes the applied field, and it is roughly proportional to the magnetization of the sample. The demagnetizing field is assumed, as in the ABBM model, to be of the form $-k m$, uniform and proportional to the magnetization, where the demagnetizing factor $k$ takes into account the geometry of the sample. From micro-magnetic calculations it can be shown that the assumption of a uniform demagnetizing field is strictly correct only in ellipsoidal geometries, however, it turns out to be a good approximation also in more general cases.

The addition of the demagnetizing field to the equation of motion has a crucial effect on the dynamics, since it provides the restoring force needed to keep the interface constantly at criticality. Without this term the system would be critical just at a threshold value of the applied magnetic field, and experience a true depinning transition, while, in BN experiments, criticality is observed in an entire range of values of $H(t)$ around the coercive field. These range of values correspond to the linear region of the hysteresis loop, which is precisely where the magnetization reversal is dominated by domain wall motion. 




(b)

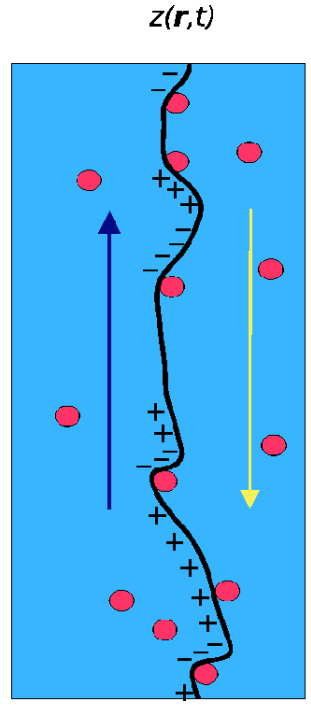

Figure 21. (a) Free magnetic charges on the boundary of the sample.(b) Free magnetic charges on the interface.

The drastic effect of the demagnetizing field on the behavior of the system is confirmed by performing experiments in particular geometries, as Barkhausen measurements on toroidal samples. In this case closed interfaces can be forced so that, due to the absence of free magnetic charges on the boundaries, the demagnetizing factor is zero, and indeed, a true depinning transition is observed. This behavior has first been observed on a FeSi single crystal in a frame geometry [76], and later on several other materials [100-102]. In any other geometry, where $k \neq 0$, the equation of motion for the interface has to be modified with respect to equation (17) by replacing the applied force $F(t)$ not just with the applied field $H(t)$, but with an effective field

$$
H_{e f f}(t)=H(t)-k m(t)
$$

The demagnetizing field produces the feedback mechanism that guarantees the presence of criticality without any tuning of the driving field. The mechanism is the following: the interface is stuck as long as $H(t)$ is such that $H_{\text {eff }}(t)$ is below the critical value $H_{c}$. As soon as $H_{\text {eff }}$ exceeds the critical value for the depinning transition, the interface starts to move in a series of discontinuous jumps. The displacement of the interface, however, causes an increase in the magnetization $m(t)$, and therefore in the demagnetizing field, so that at some point the effective field is back below the threshold value, and the avalanche stops. 
The work by Urbach et al. [98] represents a fundamental step towards the realistic description of an elastic magnetic interface, since the introduction of the demagnetizing field changes dramatically the behavior of the model, from one that shows criticality at the pinning threshold, to one that is critical in a finite range of values of the applied field.

However, the equation used in [98] to describe the domain wall dynamic still does not take into account all magnetic interactions, namely, it neglects the possible presence of free magnetic dipoles on the wall (see figure 21 (b)). Indeed, the local curvature of the interface may give rise to discontinuities in the normal component of the magnetization, and generate dipolar forces originating in the bulk of the material.

This complicated interaction has been worked out and included in the model by Cizeau, Zapperi, Durin, and Stanley [56,79]. Cizeau et al. considered the effect of dipolar forces originating from free magnetic charges on the wall, and evaluated this bulk effect, showing that it gives rise to a further contribution to the total applied force, acting as a long range elasticity, which works to flatten the interface.

The long range elasticity term turns out to be of the form:

$$
\int d \vec{r}_{1} K\left(\vec{r}-\overrightarrow{r_{1}}\right)\left(z\left(\vec{r}_{1}\right)-z(\vec{r})\right)
$$

with a kernel $K$ given by

$$
K(\vec{r})=\frac{K_{0}}{|\vec{r}|^{3}}\left(1-\frac{3 x^{2}}{|\vec{r}|^{2}}\right)
$$

This interaction has been calculated under the mild assumption that the surface charges are not strong enough to deviate the magnetization from the easy axis. The coefficient $K_{0}$ is given by $K_{0}=\mu_{0} m_{s}^{2} / 2 \pi$, where $\mu_{0}$ is the vacuum permeability, and $m_{s}$ is the saturation magnetization per unit volume.

The full equation of motion, including the contributions from surface and bulk dipolar interactions finally results to be:

$\Gamma \dot{z}(\vec{r}, t)=\nu \nabla^{2} z(\vec{r}, t)+\int d^{2} r_{1} K\left(\vec{r}-\overrightarrow{r_{1}}\right)\left(z\left(\overrightarrow{r_{1}}\right)-z(\vec{r})\right)+H(t)-k \bar{z}(t)+\eta(z, \vec{r})$,

where $\bar{z}(t)$ denotes the average position of the interface and is proportional to the magnetization.

The long range elasticity term is relevant in a renormalization group sense, since it is dominating the surface tension elastic term, as follows by power counting: rescaling $x \rightarrow x^{\prime}=b x$ and assuming that $h \rightarrow h^{\prime}=b^{\alpha} h$ with 
some positive $\alpha$, the elastic term from surface tension is rescaled by a factor $b^{\alpha-2}$, while the long range elasticity term from dipolar interactions is rescaled by $b^{d+\alpha-3}$, where $d-1$ is the dimension of the substrate plane. This point is treated in a more rigorous way in [56] where the authors, following the functional renormalization group developed in [87,92,103], obtain the value $d_{c}=2+1$ for the upper critical dimension. The lowering of the upper critical dimension of the system from $4+1$ (which is the one characterizing a generic elastic interface in a random media i.e. without the long range kernel) to $2+1$ as a consequence of the presence of the long range dipolar interactions ensures that the mean field approximation holds down to $d=3$, and ultimately allows to describe most experimental situations in terms of a mean field theory.

Corrections to scaling may however be present in $d=2+1$ : indeed the mean field power law behaviors are usually modified at the upper critical dimension by multiplicative logarithmic factors, as it is for example in the simplest case of the equilibrium Ising model in $d=4$ [104]. As an example closer to the system described by Eq. 23, let us mention that logarithmic corrections at the upper critical dimension for the depinning transition have recently been calculated for the quenched Edward Wilkinson equation with a driving force (17) in [105].

Equation (23) contains all the energetic contributions involved in the domain wall dynamics. In principle, one should add an additional noise term to the equation, to account for thermal fluctuations. However, it has been observed experimentally [98] that temperature does not affect Barkhausen measurements in bulk materials. At this regard see also [56], where a rough estimate of the signal produced by thermal effects is given, which results to be well below the typical instrumental background noise. This could be different in thin films, where thermal activated motion has been observed. Recent experiments by Kim, Choe, and Shin $[106,107]$ report measures by magneto optical imaging on $C o$ thin films, where the applied field is raised just below the coercive field and then kept constant, so that the observed Barkhausen jumps are only triggered by thermal noise (note that the Barkhausen jumps going outside the imaging window makes unreliable the estimates of the critical exponents obtained in [106,107], as already observed in [5]). therefore, in the case of thin films, an extra time dependent random term may be required in the equation of motion to include thermal noise.

The elastic interface model described by equation (23) has been extensively studied by numerical simulations, by direct integration and by simulating an automaton version of the model, and both in finite dimensions and in the mean field limit. Simulations in the infinite range limit confirm the equivalence with the ABBM model (see below), by reproducing the same amplitude distribution, and pulses statistics. The simulations of the interface model in $d=3$, recover the mean field results, in agreement with $d_{c}=3$ being the upper critical 
dimension. However, the scaling with $k$ of the cut-off in the distributions of pulses sizes and durations does not agree with the predictions of the mean field. This point will be discussed more in detail in subsection 4.10,

Although in this review we are dealing only with soft magnetic materials in the long range universality class, it is worth to note that the model by Cizeau et al. [79] has a more general validity. The results discussed above change when the long range kernel in equation (23) is absent, and the surface term is the dominating term. In this case the model is equivalent to the one previously proposed in [98]. The upper critical dimension in this case is $d_{c}=4+1$, and simulations in $d=3$ give $\tau \simeq 1.3$ and $\alpha \simeq 1.45$ for the critical exponents characterizing the pulses' distributions [108-110]. Simulations results are confirmed by renormalization group calculations $[87,108,111]$ which give $\tau \simeq 1.25$ and $\alpha \simeq 1.43$. In this case, no dependence on the driving field rate $c$ is observed. These results are in very good agreement with experiments on materials in the short range universality class $[56,112]$, indicating that the magnetic elastic interface model may indeed have a very general validity, providing a description for the magnetization reversal process suitable to deal with both universality classes of soft ferromagnets.

\subsection{The ABBM model as a mean field theory}

A mean field version of the model by Cizeau et al. [79] has been worked out by the same authors in [56], where they show that in the mean field approximation, their interface model coincides with the phenomenological ABBM model. This fact might indeed explain why the ABBM model describes the experiments so accurately.

Mean field theory is derived by taking the limit of long range interactions in the equation of motion. This is done by coupling all the points on the interface uniformly with the average wall position. Equation (23) become

$$
\Gamma \dot{z}(\vec{r}, t)=J(\bar{z}(t)-z(\vec{r}, t))+H(t)-k \bar{z}(t)+\eta(z, \vec{r}),
$$

where the coefficient $J$ includes the contributions from both short and long term elasticity. By averaging this equation over $\vec{r}$, one gets an equation for the average position of the interface:

$$
\Gamma \dot{\bar{z}}(t)=H(t)-k \bar{z}(t)+\frac{1}{S} \int d^{2} r \eta(z(\vec{r}, t), \vec{r})
$$

where $S$ is the surface of the substrate plane. The integral over the noise gives rise to an effective pinning $W(\bar{z})$, which depends only on the average position of the interface. 
Indeed from equation (18)

$$
\eta(z(\vec{r}, t), \vec{r})=\int d z \delta(z-z(\vec{r}, t)) \eta(z, \vec{r})=-R \sum_{i} \delta^{2}\left(\vec{r}-\vec{r}_{i}\right) \delta\left(z(\vec{r}, t)-z_{i}\right)
$$

which is proportional to the number of defects points that are pinning the interface to the average position $\bar{z}$. The effective noise $W$ is then given by

$$
W(\bar{z})=\frac{1}{S} \int d^{2} r \eta(z(\vec{r}, t), \vec{r})=-\frac{R}{S} \sum_{i} \delta\left(z(\vec{r}, t)-z_{i}\right) .
$$

To calculate the correlations, note that, in the difference $W(\bar{z})-W\left(\bar{z}^{\prime}\right)$, the only terms that contribute to the sum are those relative to the pinning points that have actually moved, whose number is proportional to the average wall displacement $\bar{z}-\bar{z}^{\prime}$, so that, $\eta$ being random and uncorrelated, the effective noise is a Brownian noise in $\bar{z}$ :

$$
\left\langle\left(W(\bar{z})-W\left(\bar{z}^{\prime}\right)\right)^{2}\right\rangle=D\left(\bar{z}-\bar{z}^{\prime}\right) .
$$

This finally explains the nature of the effective Brownian correlated pinning field, which was observed experimentally in [77,78], and used as an assumption in [31] to derive the ABBM equation. The long range correlations observed in the pinning field are not a result of real long range correlations between defects in the material. Rather, the effective correlated pinning field experienced by the center of mass of the wall originates as a collective effect from the interactions along the elastic wall moving in an uncorrelated disordered medium.

The average wall position is proportional to the magnetization, so that equation 25 can be rewritten as

$$
\Gamma \dot{m}(t)=H(t)-k m(t)+W(m),
$$

which coincides with the ABBM equation (14).

The ABBM model is extremely successful in describing BN experiments, although it was proposed on phenomenological basis, without a clear reference to the underlying physics. The fact that the ABBM model can be derived as a mean field theory of an interface model obtained by taking into account all the relevant magnetic interactions is therefore very important. We want to stress that the relation with the elastic interface model not only validates the ABBM model, by giving to it a more grounded microscopical derivation, but also allows to relate the model parameters to microstructural properties of the material, making possible a close quantitative comparison between theory and experiments. 


\section{Mapping $\mathrm{ABBM}$ onto a random walk process: exact results}

The ABBM approach allows to write a simple equation of motion for the center of mass of the magnetic wall separating two regions of opposite magnetization. Interestingly, the statistical properties of this dynamics can be fully worked out analytically.

The easiest way to do this is to transform the equation by eliminating the correlated noise. We show below that equation (14) maps onto an standard Langevin equation for a Brownian motion in a suitable potential. The equation has to be solved taking care of the boundary conditions, that ensure that the signal always stays positive.

\subsection{From the ABBM equation to the Langevin equation for a biased random walk in a logarithmic potential}

Equation (29) takes a particularly simple form in terms of velocity versus magnetization. Taking a derivative with respect to time, and defining $v=$ $d m / d t$

$$
\Gamma \frac{d v}{d m}=\frac{\dot{H}(t)}{v}-k+\frac{d W}{d m} .
$$

where we used $d / d t=v d / d m$.

Assuming that the field $H(t)$ is varied at constant rate $c$, as in most $\mathrm{BN}$ experiments, and defining $w(m)=d W / d m$, we get

$$
\Gamma \frac{d v}{d m}=\frac{c}{v}-k+w(m),
$$

where the noise term, being the derivative of a Brownian noise, is delta correlated in magnetization, with $\left\langle w(m) w\left(m^{\prime}\right)\right\rangle=2 D \delta\left(m-m^{\prime}\right)$.

Note that, the ABBM is defined with the assumption that just one single and rigid wall is present. This allows the identification of the magnetization with the coordinate defining the wall position, and therefore the variable $v$, which is the derivative of the magnetization with respect to time, can be interpreted both as the average velocity of the interface, and as the induced voltage directly measured in inductive $\mathrm{BN}$ experiments.

The four parameters in equation (29) can be reduced to two by rescaling the velocity $\tilde{v}=v \Gamma / D$, and defining $\tilde{k}=k / D, \tilde{c}=c \Gamma / D^{2}$, and $\eta=w / D$, so that $\left\langle\eta(m) \eta\left(m^{\prime}\right)\right\rangle=2 \delta\left(m-m^{\prime}\right)$ :

$$
\frac{d \tilde{v}}{d m}=\frac{\tilde{c}}{\tilde{v}}-\tilde{k}+\eta(m)
$$


This leaves two independent parameters $\tilde{k}$, and $\tilde{c}$. In order to avoid cumbersome notations we will avoid the tildes in the following.

Interpreting the velocity $v$ as a coordinate $x$ and the magnetization as time $t$, this is the Langevin equation for a random walk in a logarithmic plus linear potential $U(x)$ :

$$
U(x)=-c \log x+k x,
$$

where the coefficients of the two terms in the potential, that rules the dynamics, correspond to rescaled driving field rate and demagnetizing coefficient respectively.

The Fokker-Planck equation corresponding to the Langevin equation

$$
\dot{x}=\frac{c}{x}-k+\eta(t) .
$$

is

$$
\frac{\partial P(x, t)}{\partial t}=\frac{\partial}{\partial x}\left(\frac{\partial P(x, t)}{\partial x}+\left(k-\frac{c}{x}\right) P(x, t)\right)
$$

where $P(x, t)$ is the probability for the walker to be at $x$ at time $t$.

The first thing that can easily be done is to solve equation 35 in the stationary $t \rightarrow \infty$ limit. This gives

$$
P_{\infty}(x) \propto x^{c} \exp (-k x)
$$

Expression 36 gives the time independent probability distribution for the process for all values of $c$, however, it will become clear further on that, once the boundary conditions are taken into account, the signal distribution can be obtained by simply taking the asymptotic solution, as has been done here, only in the high driving rate regime (large $c$ ). We will come back to this distribution in the next section.

\subsection{The Fokker-Planck equation for a random walk in a logarithmic potential confined to the semiplane}

The linear term in the potential (33) acts as a constant bias to the random walk, that, as we will see, has the effect to introduce a cut-off in the return times distribution. To proceed, we forget for now about the bias term, and 
focus on the behavior of an unbiased random walk in a logarithmic potential:

$$
\dot{x}=\frac{c}{x}+\eta(t) .
$$

Interestingly, the same equation (37) in two dimensions appears in the analysis of the annihilation dynamics of vortex-antivortex pair in the two dimensional $X Y$ model, and has been studied in that context by Bray in [33], which we follow in the next sections for what concerns the calculation of the persistence properties of the process.

The Fokker-Planck equation associated to the Langevin equation (37) is:

$$
\frac{\partial P(x, t)}{\partial t}=\frac{\partial}{\partial x}\left(\frac{\partial P(x, t)}{\partial x}-\frac{c}{x} P(x, t)\right)
$$

where $P(x, t)$ is the probability for the walker to be at $x$ at time $t$.

Since by definition the avalanche ends when the velocity is back to zero, we are interested to the solutions of this equation with absorbing boundary conditions at the origin $x=0$ :

$$
P(0, t)=0 \text {. }
$$

Given the absorbing condition, a walk starting at $x=0$ would never leave the origin, thus we choose as initial condition for the walk a small $\epsilon<<1$ :

$$
P(x, 0)=\delta(x-\epsilon)
$$

and take the limit of vanishing $\epsilon$ at the end.

The solution of equation (38) can be searched by separation of variables [33] in the form

$$
P(x, t)=r(x) \exp \left(-k^{2} t\right)
$$

where $r(x)$ has to satisfy

$$
x^{2} r^{\prime \prime}(x)-c x r^{\prime}(x)+\left(c-k^{2} x^{2}\right) r(x)=0 .
$$

Equation (42) can be reduced to the Bessel equation by the change of variable $r(x)=x^{a} R(x)$ :

$$
x^{2} R^{\prime \prime}(x)+(2 a-c) x R^{\prime}(x)+\left((a-1)(a-c)+k^{2} x^{2}\right) R(x)=0,
$$


by choosing for $a$ the value $a=(1+c) / 2$ :

$$
x^{2} R^{\prime \prime}(x)+x R^{\prime}(x)+\left(k^{2} x^{2}-\nu^{2}\right) R(x)=0,
$$

with $\nu=((1-c) / 2)$.

The general solution of equation (38) can therefore be written as a linear combination of Bessel functions as:

$$
P(x, t)=x^{1-\nu} \int_{0}^{\infty} d k\left(\alpha(k) J_{\nu}[k x]+\beta(k) J_{-\nu}[k x]\right) \exp \left(-k^{2} t\right) .
$$

The coefficients $\alpha(k)$ and $\beta(k)$ are fixed by imposing the initial condition equation (40), that can be enforced by using the orthogonality properties of the Bessel functions:

$$
\int_{0}^{\infty} d k k J_{\alpha}\left[k x_{1}\right] J_{\alpha}\left[k x_{2}\right]=\delta\left(x_{1}-x_{2}\right) / x_{1} .
$$

This gives

$P(x, t \mid \epsilon, 0)=\epsilon\left(\frac{x}{\epsilon}\right)^{1-\nu} \int_{0}^{\infty} d k k\left(A J_{\nu}[k \epsilon] J_{\nu}[k x]+B J_{-\nu}[k \epsilon] J_{-\nu}[k x]\right) \exp \left(-k^{2} t\right)$,

where $A$, and $B$ are numerical coefficients. Finally, by performing the integrals

$$
P(x, t \mid \epsilon, 0)=\frac{\epsilon}{t}\left(\frac{x}{\epsilon}\right)^{1-\nu} \exp \left(-\frac{x^{2}+\epsilon^{2}}{4 t}\right)\left(A I_{\nu}\left[\frac{\epsilon x}{2 t}\right]+B I_{-\nu}\left[\frac{\epsilon x}{2 t}\right]\right)
$$

where $I_{\nu}[z]$ is the modified Bessel function.

To simplify the notations let us rewrite equation (48) as

$$
P(x, t \mid \epsilon, 0)=A P_{\nu}(x, t \mid \epsilon, 0)+B P_{-\nu}(x, t \mid \epsilon, 0)
$$

and analyze the two terms separately.

For what follows is useful to introduce the probability current

$$
j(x, t)=-\left(\partial_{x} P-c P / x\right)
$$

so that $\partial_{t} P=-\partial_{x} j$. The absorbing boundary condition equation (39) corresponds to requiring a negative current at the origin.

In order to evaluate the probability current at the origin corresponding to the two terms in equation (49), it is convenient to expand the Bessel functions 
in the limit of small $\epsilon$, using

$$
I_{\nu}[z]=(z / 2)^{\nu} \sum_{m=0}^{\infty}(z / 2)^{2 m} /(m ! \Gamma[m+\nu+1]) .
$$

The first term in equation (48) simplifies to

$$
P_{\nu}(x, t \mid \epsilon, 0)=\frac{4}{\Gamma[1+\nu]}(4 t)^{-(1+\nu)} \epsilon^{2 \nu} x \exp \left(-\frac{x^{2}}{4 t}\right)\left(1+O\left(\epsilon^{2}\right)\right) \text {. }
$$

The corresponding probability current is

$j_{\nu}(x, t \mid \epsilon, 0)=\frac{4}{\Gamma[1+\nu]}(4 t)^{-(1+\nu)} \epsilon^{2 \nu}\left((c-1)+\frac{x^{2}}{t}\right) \exp \left(-\frac{x^{2}}{4 t}\right)\left(1+O\left(\epsilon^{2}\right)\right)$,

and gives a non-vanishing contribution at the origin, which is negative, as requested for an absorbing boundary, as long as $c<1$.

The second term in equation (48) simplifies to

$$
P_{-\nu}(x, t \mid \epsilon, 0)=\frac{4}{\Gamma[1-\nu]}(4 t)^{-(1-\nu)} x^{1-2 \nu} \exp \left(-\frac{x^{2}}{4 t}\right)\left(1+O\left(\epsilon^{2}\right)\right) .
$$

The coefficient $1-2 \nu-c$ of the leading term of order $x^{-2 \nu}$ in the probability current vanishes, and the next-to-leading term gives:

$$
j_{-\nu}(x, t \mid \epsilon, 0)=\frac{4}{\Gamma[1-\nu]}(4 t)^{-(1-\nu)} \frac{x^{2-2 \nu}}{t} \exp \left(-\frac{x^{2}}{4 t}\right)\left(1+O\left(\epsilon^{2}\right)\right) .
$$

Since $c$ is a positive quantity, the exponent $2-2 \nu=1+c$ is always positive in the region of parameters of physical interest, and the contribution of the term $P_{-\nu}$ to the probability current at the origin vanishes. Therefore the physically relevant solution to our problem, satisfying the absorbing boundary condition is $P_{\nu}$, and we take $P=P_{\nu}$ and drop the $\nu$ sign from now on.

Finally, we can conclude that the total probability current at $x=0$ is:

$$
j(0, t)=-\frac{4 A(1-c)}{\Gamma[(3-c) / 2]}(4 t)^{-(3-c) / 2} \epsilon^{1-c}
$$

where we have replaced $\nu$ with its expression as a function of $c$. The current goes to zero as $c$ approaches 1, correctly suggesting that, as we will see more clearly in section 4.5, $c=1$ separates two different regimes. 
It must be stressed that for $c<1$ the probability $P(x, t \mid \epsilon, 0)$ in equation (52) is not normalized to unity, when integrated over $x$. Indeed, due to the absorbing boundary, part of the probability density flows to the origin, so that the conserved quantity is

$$
\int_{0}^{\infty} d x P(x, t \mid \epsilon, 0)+P_{a b s}(t \mid \epsilon, 0)=1
$$

where $P_{a b s}(t \mid \epsilon, 0)$ is the probability that the random walk has been absorbed at some time $t^{\prime}<t$. This probability goes to 1 as $t \rightarrow \infty$. For this reason, the overall time independent process amplitude distribution cannot be obtained by taking the $t \rightarrow \infty$ in $P(x, t \mid \epsilon, 0)$, since the asymptotic distribution is zero for any $x \neq 0$.

The asymptotic distribution clearly is not the correct quantity to be compared with the signal amplitude distribution in equation (5). A more meaningful probability, which indeed corresponds to the amplitude measured in experiments is obtained by integrating $P(x, t \mid \epsilon, 0)$ over $t$. In general, for a stationary process, the asymptotic distribution $P_{\infty}(x)$ coincides with the average over time of the probability $P(x, t)$ :

$$
P_{\infty}(x)=\lim _{T \rightarrow \infty} \frac{1}{T} \int_{0}^{T} d t P(x, t) .
$$

However, the quantity in equation (5) that is measured in experiments is the average over many avalanches of the probability for the signal to have a given value. During the avalanche, the process described by equation 34 is nonstationary, and thus the integral over $t$ cannot be replaced by the asymptotic distribution.

This point needs a further remark. In subsection 2.3 we stressed the fact that the BN experiments has to be restricted to the region where the signal is stationary, and this may generate confusion when compared with the present argument. The BN process is described by the stochastic equation (30) only when $v>0$, that is as long as the effective field (applied field diminished by the demagnetizing field) overcomes the pinning field. As long as $v>0$ the magnetization increases, causing a variation in the effective field, which decreases until it goes somewhere below the critical depinning threshold. At this point, the interface stays locked, until the applied field brings the effective field again above threshold. Therefore while $v=0$, the process is just deterministic. Given that the wall is at rest, there is no noise term involved. The stationarity of the whole BN process (the random process at $v>0$ plus the deterministic one for $v=0$ ) should not be confused with the stationarity of the process described by equation 34, which, in the low driving field rate 
regime, is reached only when the random walk is absorbed by the boundary.

Let us now go back to our problem of evaluating the signal amplitude distribution. By taking integral over $t$ in equation (52) to the first order in $\epsilon$ we can extract the small $x$ power law behavior:

$$
\int_{0}^{\infty} d t P(x, t \mid \epsilon, 0) \simeq x^{2 \nu+1}=x^{c}
$$

This expression is not valid for large $x$, where the small $\epsilon$ expansion does not hold. The power law dependence on $x$ of the process amplitude coincides with that of equation (36) obtained in the previous section, apart from the exponential cut-off depending on the bias term, and which has been neglected here. Indeed equation (36) gives an expression for the process amplitude distribution correct for all values of $c$.

The large $t$ asymptotic has been used in literature [109] to explain the experimental power law in equation (5), however, we stress that this behavior cannot be obtained in general by simply taking the asymptotic solution.

To compare this result with the experimental one on the distribution of the Barkhausen signal we must remember that here $t$ corresponds to the physical variable $m$, and $x$ to the wall velocity, or BN amplitude $v$. Going back to physical time will therefore produce an extra $v^{-1}$ factor, recovering the $c-1$ exponent found experimentally (see equation (5)) ).

\subsection{Distributions of sizes and durations of pulses from persistence properties}

Let us now turn to the analysis of the statistical properties of the signal pulses. The distribution of the sizes and durations of Barkhausen pulses can be derived from the persistence properties of the process (34).

Let us introduce the first passage time distribution $P_{0}(0, t \mid \epsilon, 0)$, i.e. the probability that a random walk starting at some threshold value $\epsilon$ at time $t=0$ goes back to $\epsilon$ for the first time at time $t$, and the persistence probability $P_{1}(t \mid \epsilon, 0)$, defined as the probability that a random walk that starts at $\epsilon$ at time $t=0$ stays positive up to time $t$.

These two quantities are related by the following relation:

$$
P_{1}(t \mid \epsilon, 0)=\int_{t}^{\infty} d t^{\prime} P_{0}\left(t^{\prime} \mid \epsilon, 0\right)
$$

since the probability for a random walk to be positive up to time $t$ is equal to the probability that it comes back to 0 for the first time at some later time $t^{\prime}>t$, which is given by the r.h.s. of equation (60). 
The persistence probability $P_{1}(t \mid \epsilon, 0)$ is also related to the solution of the Fokker Planck equation with absorbing boundary condition by

$$
P_{1}(t \mid \epsilon, 0)=\int_{0}^{\infty} d x P(x, t \mid \epsilon, 0),
$$

since, to survive up to some time $t$, a walker must be found at time $t$ at some positive value $x$. This is equivalent to the normalization condition (157) where $P_{a b s}(t \mid \epsilon, 0)=1-P_{1}(t \mid \epsilon, 0)$.

Using the previous two equations we can calculate $P_{0}$ as follows:

$$
P_{0}(t, \mid \epsilon, 0)=-\partial_{t} P_{1}(t \mid \epsilon, 0)=-\int_{0}^{\infty} d x \partial_{t} P(x, t \mid \epsilon, 0),
$$

where we passed the derivative under the integral.

Rather than replacing $P(x, t \mid \epsilon, 0)$ with its explicit expression and then dealing with the complicated integral, it is convenient to replace the integrand on the r.h.s. with its expression given by the Fokker-Planck equation:

$$
P_{0}(t, \mid \epsilon, 0)=-\int_{0}^{\infty} d x \partial_{x}\left(\partial_{x} P(x, t \mid \epsilon, 0)-\frac{c}{x} P(x, t \mid \epsilon, 0)\right)=j(0, t)
$$

where, in replacing the integral with the current in zero we use the fact that $P(x, t \mid \epsilon, 0)$ and its derivatives decay exponentially to zero for $x \rightarrow \infty$. Eq. (63) is consistent with the probability $P_{a b s}$ for the process to having being absorbed before time $t$ being equal to the total current flow through the origin up to time $t: P_{a b s}=-\int_{0}^{t} j(0, s) d s$.

Finally we get from equation (56):

$$
P_{0}(t, \mid \epsilon, 0) \propto(1-c) t^{-(3-c) / 2} .
$$

When $c \rightarrow 0$ equation 64 gives back the well known result for the persistence probability of a free random walk.

To see how this relates to the distribution of sizes and durations of BN pulses one has to go back to the physical variables, where $t$ has to be interpreted as magnetization. Equation (64) gives the probability that the avalanche ends at some given value of the magnetization. It gives therefore directly the distribution of sizes of the avalanches, which is $p(S) \sim S^{-(3-c) / 2}$. This gives the correct exponent $\tau=3 / 2$ in the quasi-static limit $c=0$, and the expected linear dependence on $c$ for finite driving field rates.

The distribution of durations of BN pulses can be also derived by this same result, by imposing that $p(S) d S=p(T) d T$, and using the scaling $S \sim T^{2}$ 
that we will show in subsection 4.7. This gives $p(t) \sim T^{-(2-c)}$, with $\alpha=2$ for vanishing field rate, and a linear dependence on $c$ that agrees with the fit of experimental histograms. Note that the exponential cut-offs cannot be recovered from this analysis, since they involve the demagnetizing factor that has been put to zero here (see beginning of section 4.2).

\subsection{Non-universality of the exponents and marginality of the logarithmic potential}

The continuous dependence on a parameter in otherwise universal exponents is usually related to the presence of some potential which is marginal in the renormalization group sense. Indeed, also in the present case, the dependence of the exponents $\alpha$ and $\tau$ on the driving field rate $c$ can be attributed to some marginal contribution.

This becomes clear in the mapping onto the random walk process. The non-universality of the exponents in the persistence and first passage time probabilities are special to the logarithmic potential, and are related to the marginality of the $1 / x$ perturbation to a free random walk in a renormalization group sense. Indeed, analyzing the relevance of a power law perturbation $x^{\alpha}$ to a free random walk, one can show by simple scaling arguments that the force is irrelevant for $\alpha>-1$ (the random walk behaves essentially as if it where free), while it is relevant, and dominates the noise term for $\alpha<-1$ (the motion is in many respects deterministic, and persistence properties are governed by rare fluctuations of the noise).

The equation for a random walk in a generic power law potential is

$$
\dot{x}=c x^{-\psi}+\eta(t) .
$$

Under rescaling $x \rightarrow a x, t \rightarrow a^{z} t$, the coefficients rescale as $c \rightarrow a^{z-1-\psi} c$, $D \rightarrow a^{z-2} D$, where $D$ is the noise correlation. The equation of motion therefore becomes

$$
\dot{x}=c a^{z-1-\psi} x^{-\psi}+a^{z / 2-1} \eta(t) .
$$

This equation has two fixed points, depending on the value of $\psi$. The potential term dominates with respect to the noise term for $z-1-\psi>z / 2-1$. In this case, the noise term renormalizes to zero, and the value of the dynamical exponent is obtained by balancing the potential term with the l.h.s., which gives $z=1+\psi$. Replacing the fixed point value of $z$ in the inequality we deduce that this is the fixed points for $\psi<1$.

The other regime is the one where the noise dominates over the potential, for $z / 2-1>z-1-\psi$. In this case, the coefficient of the potential renormalizes 
to zero, and the value of the dynamical exponent is obtained by balancing the noise with the l.h.s., which gives $z=2$. Replacing the value of $z$ in the inequality we verify that the system renormalize to this fixed point as long as $\alpha>1$.

Therefore, for $\psi>1, z=2$, and $c$ rescales to zero: the force is irrelevant, and the random walk behave essentially as if it were free. For $\psi<1, z=1+\psi$, the noise correlation $D$ rescales to zero: the noise term is (dangerously) irrelevant, and the process is almost deterministic.

The case of our equation, $\psi=1$, corresponding to the logarithmic potential, is marginal: it is the value of $\psi$ for which all the three terms in the equation balance, with $z=2$. This is at the origin of the non-universality of the persistence exponents and of their continuous dependence on the coefficient $c$ of the potential.

The effect of the driving field rate on crackling events has been recently studied by White and Dahmen in [55]. In this work the authors make a distinction between an avalanche (sequence of events triggered by an initial event at constant driving field), and a pulse (uninterrupted sequence of events measured in experiments). In general, at non-zero sweep rate a pulse can be a superposition of several simultaneously propagating avalanches, while avalanches and pulses coincide only in the adiabatic limit. Under some general assumptions that are fulfilled by many crackling systems, the authors of [55] show by scaling arguments that the value of the exponent $\alpha$ in the distribution of events durations determines whether or not the pulse statistic will be affected by the sweep rate $c$. In particular, they claim that no dependence on $c$ has to be expected for $\alpha<2$, while a linear dependence of the kind $\alpha=2-c$ is predicted in the case $\alpha=2$. It would be very interesting to make a connection between the results obtained in [55] in terms of superposition of adiabatic avalanches with those reported here for the ABBM model, but this has not been done yet.

\subsection{Existence of a threshold in the driving field rate from recurrence properties of the free random walk}

Another evidence in Barkhausen noise phenomenology is the fact that the intermittent behavior typical of the avalanche dynamics is lost when the driving field is cycled too fast: above a threshold value of the driving field rate the typical intermittent signal disappears, and the magnetization reversal proceeds in a unique avalanche.

The existence of a threshold value in the driving field rate $c$, corresponds to the existence of a special value $c$ for the coefficient of the logarithmic potential, that discriminates among two qualitatively different behaviors. The value $c=1$ already appeared in equation (56) as the threshold below which the probability 
current at the origin is negative.

Essentially, this value of $c$ determines whether or not the random walk is recurrent. The random walk is certainly recurrent for $c<0$, which corresponds to an attractive potential at the origin. This, however, does not correspond to the physical case of BN. The case of physical interest is $c>0$, corresponding to a repulsive potential. Interestingly, the random walk may come back to the origin even when it is subject to a logarithmic repulsion, as long as the repulsive potential coefficient is not too strong. This gives a condition on the coefficient $c$.

A a simple and clear way to interpret this behavior is to observe that there exists a natural mapping between the free random walk in $d$ dimension and the confined one dimensional random walk in a logarithmic potential. Starting from the Fokker-Planck equation for a free random walk in $d$ dimensions.

$$
\partial_{t} P(\mathbf{x}, t)=\nabla_{x} P(\mathbf{x}, t)
$$

we can write the Fokker-Planck equation for the radial part in polar coordinates as follows

$$
\partial_{t} P_{R}(r, t)=\partial_{x}\left(\partial_{x} P_{R}(r, t)-\frac{d-1}{r} P_{R}(r, t)\right) .
$$

This equation shows that the radial part of free random walk in $d$ dimensions performs a one dimensional random walk in a logarithmic potential confined to a semiplane, with coefficient $c$ equal to $d-1$ for the potential.

Using this mapping, the existence of a threshold value in the coefficient of the potential is explained in terms of the well known recurrence properties of the free random walk. The condition $d<2$ for the random walk to be recurrent translates into $c<1$.

\subsection{Cut-off in the distributions of pulses' sizes and durations as an effect of the bias to the random walk}

Let us now analyze the effect of to the bias term $k$. We consider a simple biased random walk first:

$$
\dot{x}(t)=-k+\eta(t) .
$$

By rescaling $x \rightarrow x+k t$, it is clear that the probability for a biased random walk with constant bias $k$ to return to the origin is equivalent to the probability for a free random walk to return onto the line $k t$. Given that the random walk is sub-linear, this introduces a characteristic timescale $\tau$, after which the random 
(a)

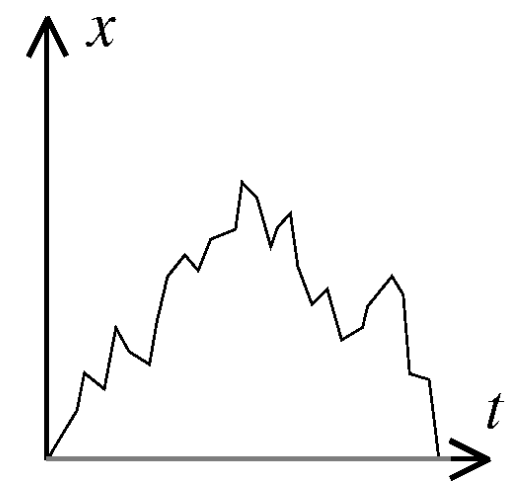

(b)

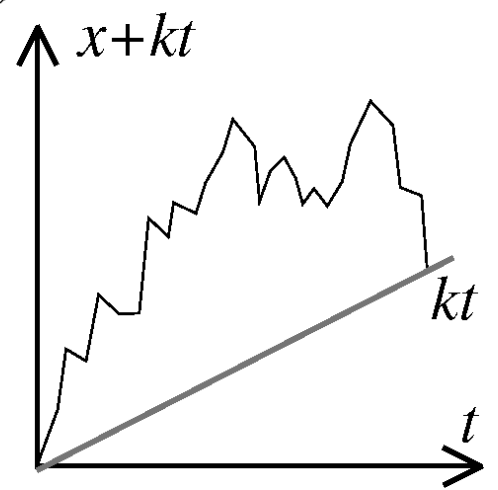

Figure 22. Sketch of the mapping of the return of the biased random walk to the origin (a) onto the return of the free random walk to the line $k t$ (b). The characteristic time $\tau$ is defined as the typical time at which the free random walk crosses the line $k t$.

walk will very unlikely, namely with exponentially small probability, go back to the line $k t$ (see figure 222).

The value of the characteristic time $\tau$ is such that $k \tau \sim \sqrt{\tau}$, which gives $\tau \sim k^{-2}$. Since $t$ corresponds to magnetization in the physical variables, $\tau$ corresponds to the characteristic avalanche size $S_{0}$ in the cut-off of equation (6). As we will see further on, $T_{0} \sim S_{0}^{1 / 2}$, giving $T_{0} \sim k^{-1}$ for the cut-off in equation (7).

As we have seen in subsection 4.4, the logarithmic potential term does not alter the dynamic exponent $z$ which is still equal to 2 as for the free random walk, thus the walk in the logarithmic potential is still sublinear, and is affected by the bias in the same way as the free random walk.

The fact that the bias term $k$ generates an exponential cut-off in the distribution of the return times, physically means that the size of the largest magnetization event is regulated by the demagnetizing field.

\subsection{Average shape of Barkhausen pulses as excursion of the random walk process}

In analogy to conventional critical phenomena one might expect that pulses of different durations have an average shape that, once properly rescaled, collapses onto a universal scaling function. In a recent paper by Sethna et al. [1] it was suggested that this scaling function is an interesting quantity to measure in order to make quantitative comparison between the theoretical models and experiments. This quantity provides a much sharper tool to test theory against experiments than the simple comparison of critical exponents, that are scalar quantities. 
Indeed, by performing this kind of analysis on experimental data, average pulses, properly rescaled by their durations, approximately collapse onto the same function (see figure 14) [113].

The average shape of the pulse is related, in terms of the corresponding stochastic process, to what is known in the mathematical literature as the excursion, namely the average trajectory between two successive return of the process to some reference value.

The average shape for the process (37) has been calculated in [113]. This work was exactly motivated by the problem, raised in [1], of finding the average form of a Barkhausen pulse as a function of time.

Let us consider a generic random walk in a potential $U(x)$ :

$$
\dot{x}(t)=U(x)+\eta(t) .
$$

The average over trajectories starting at $x=0$ at time $t=0$, and constrained to come back to $x=0$ for the first time at $t=T$, that we will denote as $\langle x(t)\rangle_{T}$ is found to scale, in appropriate time regimes depending on the potential $U$, as

$$
\langle x(t)\rangle_{T}=T^{\gamma} f(t / T)
$$

where $f$ is some scaling function $[114,115]$.

Let us consider processes constrained to the semiplane $x>0$, and focus on the returns to the origin (take as reference value a small threshold $x=\epsilon$ ). Once the solution with absorbing boundary conditions of the Fokker Planck equation corresponding to the Langevin equation (70) is known, the excursion can be expressed by averaging over all trajectories going through $x$ at time $t$.

In general, the probability for a Markovian process starting at $\epsilon$ at time 0 and going back to $\epsilon$ at time $T$ to be found at position $x$ at time $t$, is given by $c(x, t ; \epsilon, T)=P(x, t ; \epsilon, 0) P(\epsilon, T ; x, t)$. Using time translational invariance $c(x, t ; \epsilon, T)=P(x, t ; \epsilon, 0) P(\epsilon, T-t ; x, 0)=P(x, t ; \epsilon, 0) \bar{P}(x, T-t ; \epsilon, 0)$, where $\bar{P}$ is the conditional probability for the inverse process.

The average excursion is therefore obtained by taking the following limit for $\epsilon \rightarrow 0$ :

$$
\langle x(t)\rangle_{T}=\lim _{\epsilon \rightarrow 0} \frac{\int_{0}^{\infty} d x x P(x, t ; \epsilon, 0) \bar{P}(x, T-t ; \epsilon, 0)}{\int_{0}^{\infty} d x P(x, t ; \epsilon, 0) \bar{P}(x, T-t ; \epsilon, 0)},
$$

which counts the fraction of excursions that go through $x$ at time $t$.

It is instructive to calculate the excursion for the simplest case of a free random walk [116]. The probability $P(x, t ; \epsilon, 0)$ for the walk confined to the semiplane can be calculated by the image method as a linear combination of 
two solutions of the Fokker Planck for the free process:

$$
P(x, t ; \epsilon, 0)=\sqrt{\frac{2}{\pi t}}\left(\exp ^{-(x-\epsilon)^{2} / 2 t}-\exp ^{-(x+\epsilon)^{2} / 2 t}\right)
$$

which for small $\epsilon$ gives

$$
P(x, t ; \epsilon, 0)=\sqrt{\frac{2}{\pi}} t^{-3 / 2} \epsilon x \exp ^{-x^{2} / 2 t} .
$$

In this case $\bar{P}=P$ due to the process time reversal invariance, therefore the average excursion is given by

$$
\langle x\rangle_{T}=\frac{\int_{0}^{\infty} d x x^{3} \exp ^{-x^{2} / 2 \tau}}{\int_{0}^{\infty} d x x^{2} \exp ^{-x^{2} / 2 \tau}},
$$

with $\tau=t(T-t) / t$. Evaluating the integrals gives

$$
\langle x\rangle_{T}=T^{1 / 2} \sqrt{\frac{8}{\pi}} \sqrt{\frac{t}{T}\left(1-\frac{t}{T}\right)} .
$$

Therefore the exponent $\gamma$ is equal to the wandering exponent of the free process $\gamma=1 / 2$, and the scaling function is

$$
f(x)=\sqrt{8 / \pi} \sqrt{x(1-x)}
$$

normalized to have $\int_{0}^{1} d x f(x)=1$.

Let us now consider the case interesting to our theory of the excursion for the random walk in a logarithmic potential. This can be calculated in a similar way, by observing that the process is still time translational invariant, and it is invariant under $t \rightarrow-t$, as long as the coefficient $c$ is also inverted in the revers process. Therefore $\bar{P}(x, T-t ; \epsilon, 0)$ is obtained by replacing $c$ with $-c$ in $P(x, T-t ; \epsilon, 0)$.

Indeed, by repeating the previous steps with the solution of the Fokker Plank equation (38), the calculation finally reduces, also in this case, to evaluating the ratio between two successive moments of a half Gaussian with variance $w=2 t(T-t) / T$, so that the average excursion results to be proportional to $\sqrt{w}=\sqrt{t(T-t) / T}$, as in the case of the free random walk, and it is still given by equation (77).

To translate this result into the shape of the Barkhausen pulse, we have to go back to our original physical variables. The excursion calculated above 


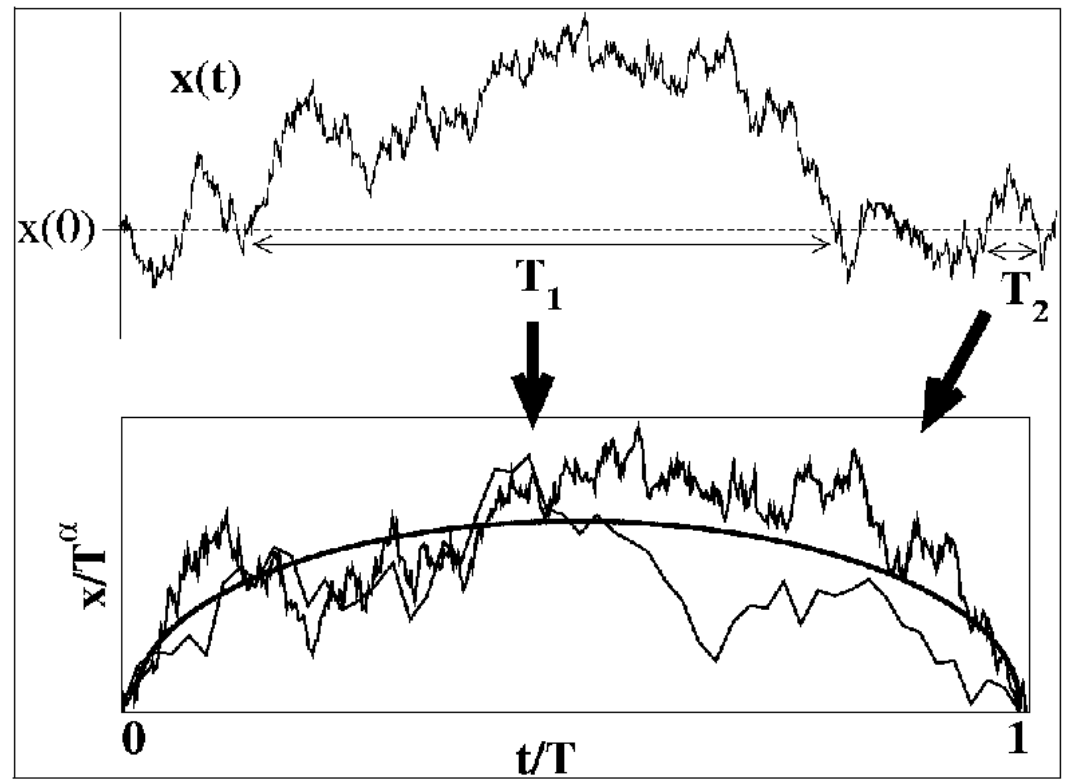

Figure 23. Schematic representation of the average excursion. Different realizations of the stochastic process all returning at the origin after the same time interval $T_{1}$ are averaged to give a smooth curve. Repeating the same process with a different return time interval $T_{2}$ gives another curve, that can eventually be rescaled onto the first.

corresponds to the signal $v$ as a function of the avalanche size $s$. The average pulse as a function of $s$ and of the total size $S$ of the pulse is then

$$
\langle v(s, S)\rangle=S^{1 / 2} f(s / S)
$$

However, the commonly measured experimental pulses, as those shown in figure 14, represent the signal $v$ as a function of duration $t$ of the avalanche, that will have some different scaling and shape

$$
\langle v(t, T)\rangle=T^{\beta} g(t / T) .
$$

Following [113] we calculate the scaling function $g$ : we have to express $s$ and $S$ as functions of $t$, and $T$. By definition, the size $s$ of the avalanche at time $t$, is the integral of the signal $v$ from time 0 to $t$ :

$$
\langle s(t, T)\rangle=\left\langle\int_{0}^{t} d t^{\prime} v\left(t^{\prime}, T\right)\right\rangle=T^{\beta+1} \int_{0}^{t / T} d x g(x),
$$


therefore, the total size $S$ scales as $S \simeq T^{\beta+1}$. Approximating $s(t, T)$ with its average value in the equation

$$
v(t, T)=v(s=s(t, T), S=S(T))
$$

gives the following condition on $g$ :

$$
T^{\beta} g(t / T)=T^{(\beta+1) / 2} f\left(\int_{0}^{t / T} d x g(x)\right) .
$$

This enforces $\beta=1$, and gives an implicit integral equation for $g$ :

$$
g(x)=f\left(\int_{0}^{x} g\left(x^{\prime}\right) d x^{\prime}\right)
$$

that has to be solved subject to the boundary conditions $g(0)=g(1)=0$. Equation (83) takes a simpler form in terms of $h(x)=\int_{0}^{x} g\left(x^{\prime}\right) d x^{\prime}$ :

$$
h^{\prime}(x)=f(h(x))=\pi \sqrt{h(x)(1-h(x))},
$$

which is solved by $h(x)=\sin ^{2}(\pi x / 2)$. This gives for $g(x)$

$$
g(x)=h^{\prime}(x)=\pi / 2 \sin (\pi x)
$$

which is normalized to unit and satisfies $g(0)=g(1)=0$. Finally, for the normalized average avalanche as a function of its duration we get:

$$
\langle v(t, T)\rangle=\frac{\pi T}{2} \sin \pi t / T
$$

Note that, while Eq. 78 with $f$ given by Eq. 77 is an exact result, and gives the scaling form of an avalanche as a function of magnetization, to derive Eq. 86 one has to replace the dependence of $s$ on time in a single realization with its average, given by Eq. 80 .

Figure 24 shows the comparison between rescaled experimental pulses of figure 14, and the scaling function (85) predicted by the ABBM. The curves have similar shapes, however, the experimental collapse is characterized by an evident leftward asymmetry, while the ABBM predicts a symmetric shape. Note that normalizing the average pulses with $N$ as done in figure 24 should 


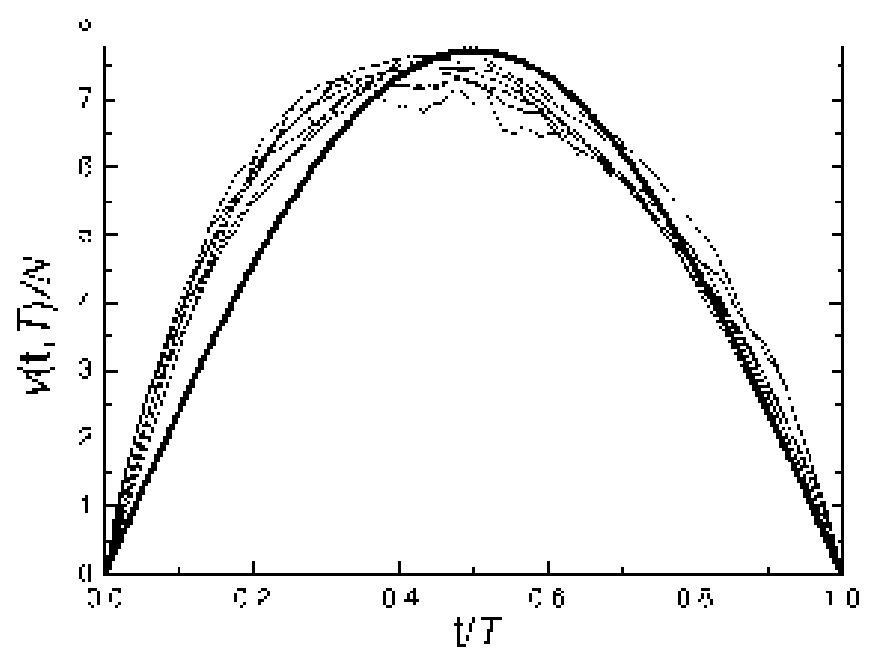

Figure 24. The approximate collapse of average pulses in figure 14 is compared with the prediction of the ABBM model (bold line) from equation 85] (Adapted from [5]).(Reprinted with permission from [5]). [5] Copyright 2005 by Academic Press.

in principle be equivalent to rescaling them by $T^{-1}$, as in equation ( $(86)$, since

$$
N=\int_{0}^{1} v(t, T) d(t / T)=S / T
$$

and $S \sim T^{2}$. However, if one directly plots $\langle v(t, T)\rangle T^{-1}$, the curve collapse is obtained only for short avalanches, while a clear deviation appears for longer avalanches (see figure 25)

This implies that the scaling $S \sim T^{2}$ does not hold for large avalanches. Indeed, by plotting the average size $\langle S\rangle$ versus $T$ for the same BN signal, the curve deviates from the predicted scaling for avalanches longer then $\sim 0.6 \mathrm{~ms}$, which is more or less where the pulses start to deviate form the expected collapse (see figure 26).

\subsection{Power spectra}

The power spectrum for the ABBM equation has been calculated in the original paper [31]. In order to get the correlation function it is more convenient to use directly the physical variables, time, magnetization, and velocity (or signal amplitude). The probability $R$ to find a signal $v$ at time $t$ is related to the probability $P$ used in the previous sections by

$$
R(v, t)=\left.P(v, m) \frac{d m}{d t}\right|_{m=m(t)}=\left.P(v, m) v\right|_{m=m(t)} .
$$






Figure 25. Average avalanche shapes for a polycrystalline FeSi material. Signals as a function of time $v(t, T)$ are scaled according to equation (86). The bold line is the theoretical prediction.

Numbers in the graph denote avalanche duration in ms. (Adapted from [113]). (Reprinted with permission from [113]). [113] Copyright 2004 Elsevier.



Figure 26. Average avalanche size as a function of its duration for polycrystalline $\mathrm{FeSi}$ material. Data are from the same experiment of figure 25 The solid line is the predicted scaling $S \sim T^{2}$. (Reprinted with permission from [5]). [5] Copyright 2005 by Academic Press.

The signal-signal time correlation function is defined as

$$
C(t)=\langle(v(t)-c / k)(v(0)-c / k)\rangle .
$$

By deriving the probability $R$ with respect to $t$ and using the Fokker Planck equation, it is easy to get a differential equation for $C$ (see [31] for the details), 
which turns out to be

$$
\frac{d C(t)}{d t}=-k C(t)
$$

Therefore $C(t)=c / k \exp (-k t)$, which corresponds to a Lorentzian power spectrum

$$
F(\omega)=\frac{2 c}{\omega^{2}+k^{2}}
$$

Equation (91) captures the correct $\omega^{-2}$ large frequency decay found in experiments, however it saturates to a constant for small $\omega$, without showing any peak.

To get a better prediction of the power spectrum, let us go back once again to the original formulation of the ABBM model, which includes a more general case, that we have not considered up to now, in which a finite correlation length $\xi_{P}$ is associated to the pinning field [31]. A finite $\xi_{P}$ has the effect of confining the pinning field, and physically measures the length over which the pinning field decorrelates.

In most cases it can safely be assumed $\xi_{P} \rightarrow \infty$, as done in most literature, and in the analysis reported in the previous sections. However, the existence of a finite correlation length in the pinning field affects the low frequency behavior of the power spectrum. The power spectrum for the case of a finite pinning correlation length is calculated in [31] in a high field velocity approximation, and results to be

$$
F(\omega)=\frac{2 c \omega^{2}}{\left(\omega^{2}+k^{2}\right)\left(\omega^{2}+\tau_{P}^{-2}\right)},
$$

where $\tau_{P}=\xi_{P} / c$ is a characteristic time associated to the correlation length $\xi_{P}$.

Equation (92) reproduces the low frequency part of the spectrum. It predicts the existence of a peak at $\omega_{M}=\left(k \tau_{P}\right)^{1 / 2} \sim c^{1 / 2}$, with a maximum value of the spectrum $F_{M}=\frac{4 c}{\left(\tau^{-1}+\tau_{P}^{-1}\right)^{2}}$ which scales as $c$ for small driving rates, as observed in experiments.

It would be interesting to understand how the presence of a finite $\xi_{P}$ affects other properties of the avalanches, as the decorrelation of the pinning field at some finite length scale might be responsible of some of the observed deviations from the $\xi_{P} \rightarrow \infty \mathrm{ABBM}$ theory in large events.

The deviation from the $1 / \omega^{2}$ prediction for the tail, which is observed at intermediate frequencies in some experiments might have the same origin as the deviation from scaling observed in the pulse shape scaling in the previous 


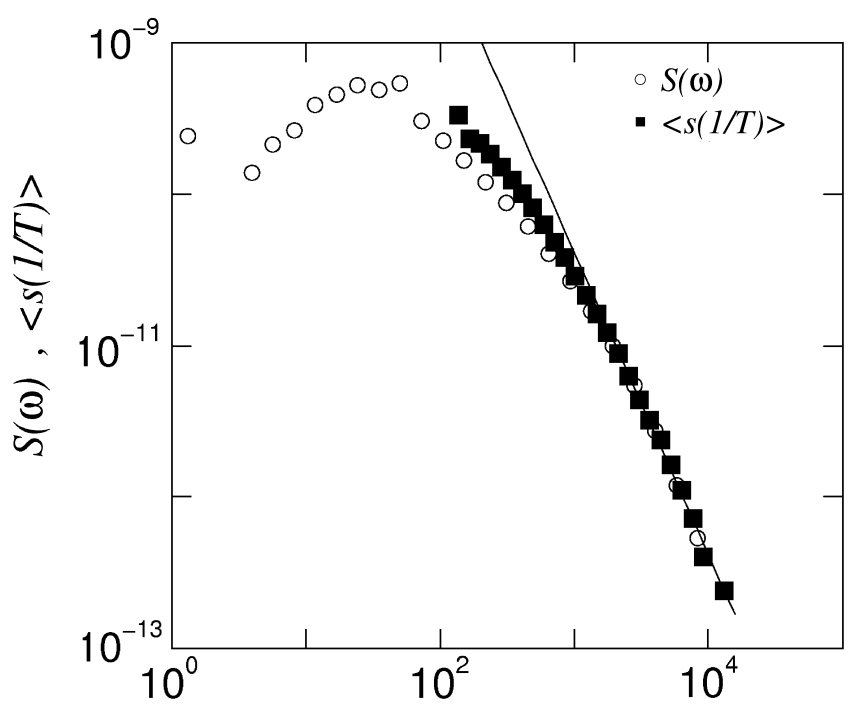

Figure 27. Comparison of the power spectrum $F(\omega)$ with the average avalanche size $\langle S\rangle$ as a function of the inverse duration for a polycrystalline FeSi material. The solid line is the predicted scaling $S \sim T^{2}$. (Adapted from [117]). (Reprinted with permission from [117]). [117] Copyright 2002 by Elsevier.

subsection [117]. This is suggested by the comparison of the power spectra with the average size versus inverse avalanche duration. The two curves deviate for the same value of $\omega$ (see figure 27). The origin of the deviation in the size versus duration plot observed in some experiments is, however, still unclear.

A possibility is that the eddy current retardation that we will discuss in the next section is responsible for the intermediate frequencies deviations from the asymptotic scaling, as suggested in [118] based on simulation of the modified $\mathrm{ABBM}$ equation. We will come back to this in the next section.

\subsection{Summary of ABBM predictions and comparison with the phenomenological observations}

Let us summarize the ABBM predictions and compare them with the phenomenological observations from $\mathrm{BN}$ experiments on soft materials with long range interactions:

(i) The distribution of the amplitude of the BN signal follows a power law decay with an exponent $1-c$ as observed in experiments (see equation 5).

(ii) The distributions of sizes and durations of avalanches can be calculated in terms of recurrence properties of the process described by equation 34 , and turn out to be power laws, with exponents $\alpha=2$ and $\tau=3 / 2$ in the quasi-static limit $c=0$, as found in experiments. 
(iii) Equation 34 predicts a linear dependence of the exponents $\alpha$ and $\tau$ on the driving rate $c$, given by $\alpha=2-c$ and $\tau=(3-c) / 2$, the same obtained in experiments.

(iv) There exist a threshold $c=1$ above which the process in equation 34 become transient. This corresponds to the threshold in the driving field rate above which the $\mathrm{BN}$ pulses collapse into a unique large avalanche of magnetization reversal.

(v) The bias term in equation 34, deriving from the demagnetizing field, is responsible for the exponential cut-offs in the distributions of sizes and durations of avalanches. The dependence on $k$ of the cut-off predicted by the ABBM equation is however different from that observed in experiments.

(vi) The average excursion of a trajectory for the stochastic process in equation 34 can be calculated and is related to the average pulse shape in BN. However, the predicted shape is symmetric, while experimental pulses show a clear leftward asymmetry.

(vii) The power spectrum decays at large frequencies as $\omega^{-2}$. A finite correlation length for the pinning field produces a peak at small frequencies at $\omega_{M} \sim c^{1 / 2}$ of amplitude $F_{M} \sim \omega$. These results are compatible with experimental ones, however, deviations from the predicted behavior are observed at intermediate frequencies in some experiments.

These predictions can be compared point to point with the experimental observations summarized in sec 2.4.9. Most of the phenomenology is captured by the ABBM model, however, two main unexplained discrepancies between experiments and theory appear: one is the scaling of the cut-offs with the demagnetizing factor $k$, the other is the presence of a leftward asymmetry in the pulse shape.

The first inconsistency can be dealt with by going back to the full elastic interface model, as we will discuss in briefly the next subsection. The second one is more serious, as the pulses asymmetry is related to non-universal properties of the magnetization reversal of microscopic origin, and we will postpone its discussion to the next section.

\subsection{Cut-off out of mean field approximation}

Let us go back for a moment to the elastic interface model discussed in section 3.4. This model has been extensively simulated both in the infinite range case, corresponding to the mean field, and in finite dimension $d$. The case $d=3$ is supposed to give results that, apart from logarithmic corrections, coincide with the mean field, since $d=3$ is the upper critical dimension for this system. This is confirmed in simulations, where one gets in both cases, for distribution 
of sizes and durations of the pulses, the exponents $\alpha$ and $\tau$ predicted by the ABBM theory, both in the adiabatic limit $c \rightarrow 0$, and for finite driving $c$.

However, the scaling with $k$ of the cut-offs $S_{0}$ and $T_{0}$ in the distributions is different in the case $d=3$ and in mean field. The simulations on the infinite range model confirm the results of section 4.6, they predict that the cut-offs scale as $S_{0} \sim k^{-2}$, and $T_{0} \sim k^{-1}$, consistently with the results obtained for the ABBM model. However simulations in $d=3$ are not compatible with these results.

Earlier simulations [56] in $d=3$ give $S_{0} \sim k^{-1}$, and $T_{0} \sim k^{-1 / 2}$, both in the quasi-static limit and at finite $c$. In [56] the full size and duration distributions are fitted by

$$
P(S, k) \sim S^{-(3-c) / 2} f_{S}\left(S / k^{-\gamma_{S}}\right),
$$

and

$$
P(T, k) \sim T^{-(2-c)} f_{T}\left(T / k^{-\gamma_{T}}\right),
$$

with $\gamma_{S} \simeq 1$, and $\gamma_{T} \simeq 1 / 2$, which are claimed to be compatible with the scaling obtained in the experiments reported in the same paper, where the cut-offs dependence on $k$ has been investigated by performing experiments on samples with different geometries. It has to be taken into account the extrapolation of a precise cut-off value is complicated by the further dependence of the distributions on the driving field rate $c$.

In more recent papers, different values are reported [54,57], corresponding to $\gamma_{S} \simeq 2 / 3$, and $\gamma_{T} \simeq 1 / 3$. These latter results are also supported by the following argument. The maximum interface jump is limited by a correlation length $\xi$, which is the length at which the dipolar interaction term, of order $\xi^{-1}$ overcome the restoring force. In $d=3$ (where the interface is not rough), this is of order $k \xi^{d-1}$, where $\xi^{d-1} h$ is the size of the avalanche. This gives $\xi \sim k^{-1 / d}$, and therefore $S_{0} \sim \xi^{d-1} \sim k^{-(d-1) / d}=k^{2 / 3}$.

In the same papers $[54,57]$, results from more recent experiments are also reported, corresponding to $\gamma_{S} \simeq 0.6$, and $\gamma_{T} \simeq 0.3$, that could be compatible with the values $\gamma_{S} \simeq 2 / 3$, and $\gamma_{T} \simeq 1 / 3$. In these works, the experimental estimates of the dependence on the demagnetizing field is extracted by repeating experiments on the same sample, which is progressively cut in order to change the value of $k$. This procedure allows to change the value of $k$ while stress and internal disorder are kept constant in the repeated experiments (see figure 13. which also reports the results of experiments on materials belonging to the short range universality class not discussed here). The experimental results in $[54,57]$ are therefore expected to be more reliable than previous ones, where different samples where used as in [56]. 
The scaling with $k$ of the cut-offs in the distributions of sizes and durations of the avalanches seems to agree with that predicted by the elastic interface model at the upper critical dimension $d=3$, although this prediction is different from the mean field one. The origin of the discrepancy between mean field behavior and the behavior at the upper critical dimension is not fully understood, and we believe that this point would still need further analysis in order to be definitely clarified.

\section{Beyond ABBM: Asymmetry in the pulse shape}

As we have seen in the previous section, despite its extreme simplicity, the ABBM model is able to capture a number of phenomenological evidences from $\mathrm{BN}$ experiments. However, the analysis on the scaling functions is more subtle: although the average pulses do approximately collapse onto each other once rescaled by their duration, by comparing the average shape of rescaled experimental Barkhausen pulses with the prediction from the ABBM model, one clearly observes a discrepancy: while the ABBM model predicts a symmetric shape, experimental pulses are systematically characterized by a visible leftward asymmetry, as can be seen in figure 24. The leftward asymmetry indicates that, unlike what would be expected from standard inertia, avalanches start fast and end slowly.

Given the success of ABBM theory in reproducing many other aspects of phenomenology, this inconsistency has been considered quite enigmatic, and the issue of understanding its origin has remained an open problem for some time.

The first attempts to identify the origin of the asymmetry have been done in [114], and more extensively in [115]. In these works, the authors pose the general problem of analyzing the behavior of the average excursion in a stochastic process, in order to understand to which extent this quantity is universal, and what kind of information it gives on the process. The idea was that this analysis could suggest in which direction the ABBM equation had to be modified in order to recover a leftward asymmetric pulse shape.

For a large class of stochastic processes, a scaling law of the form 71 is obeyed, in an appropriate time regime, with a scaling function that is, to a large extent, independent of the details of the single increment distribution, while it encodes interesting information on the presence and form of correlations. Indeed the introduction of correlated noise, is found to induce asymmetric shapes. However, in the cases considered in $[114,115]$, the scaling functions are characterized by a negative skewness, corresponding to a rightward asymmetric shape.

More recently $[118,119]$, the origin of the asymmetry has finally been identi- 
fied as a transient effect of eddy currents. Eddy currents are generated in conducting magnets as a response to domain wall displacements. This response, however, is not instantaneous and acts as an anti-inertial effect on the domain wall dynamics. It turns out that the effect of this retardation can indeed be accounted for, to a first approximation, by including a negative effective inertial term in the stochastic equation of motion for the domain wall.

\subsection{Experimental evidence of asymmetric pulses}

Figure 14 shows an attempt to collapse average pulses of different durations, obtained from a BN experiment on a polycrystalline FeSi sample, onto the same function. The pulses do approximately collapse, when properly rescaled, however, the supposedly universal shape is clearly not symmetric, as would be predicted by the ABBM model (see figure 24). The asymmetry appears to be more pronounced for small avalanches.

To get a quantitative measure of the asymmetry the skewness can be calculated as:

$$
\Sigma(T)=\frac{\frac{1}{T} \int_{0}^{T} d t\langle v(t, T)\rangle(t-\bar{t})^{3}}{\left\langle\frac{1}{T} \int_{0}^{T} d t\langle v(t, T)\rangle(t-\bar{t})^{2}\right\rangle^{3 / 2}},
$$

where $\bar{t}=\int_{0}^{T} d t\langle v(t, T)\rangle / T$.

Figure 28] shows the skewness as a function of the pulse duration in an experiment on a partially crystallized $\mathrm{Fe}_{64} \mathrm{Co}_{21} \mathrm{~B}_{15}$ ribbon. The skewness is always positive, corresponding to the leftward asymmetry. It shows a peak for $T_{P} \simeq 400 \mu s$, and decays to zero as $T$ goes to infinity.

The fact that, except for the initial increase at very small durations, the asymmetry becomes smaller and smaller for larger and larger avalanches suggests that the ingredient still missing in the ABBM theory must involve microscopic details of the system that become negligible on larger scales. The presence of a peak indicates the existence of some microscopic timescale.

\section{$5.2 \quad$ Retarded eddy current pressure}

To understand why ABBM is unable to reproduce the leftward asymmetry of the average pulse shape, we have to go back and question the assumptions which were made in its derivation. The equation of motion for the magnetic interface was derived by assuming that the work done by the total effective field (the applied field corrected by the demagnetizing and pinning fields) balances at any time the energy dissipated by eddy currents, which, in the quasistatic approximation, is proportional to the instantaneous velocity. The 


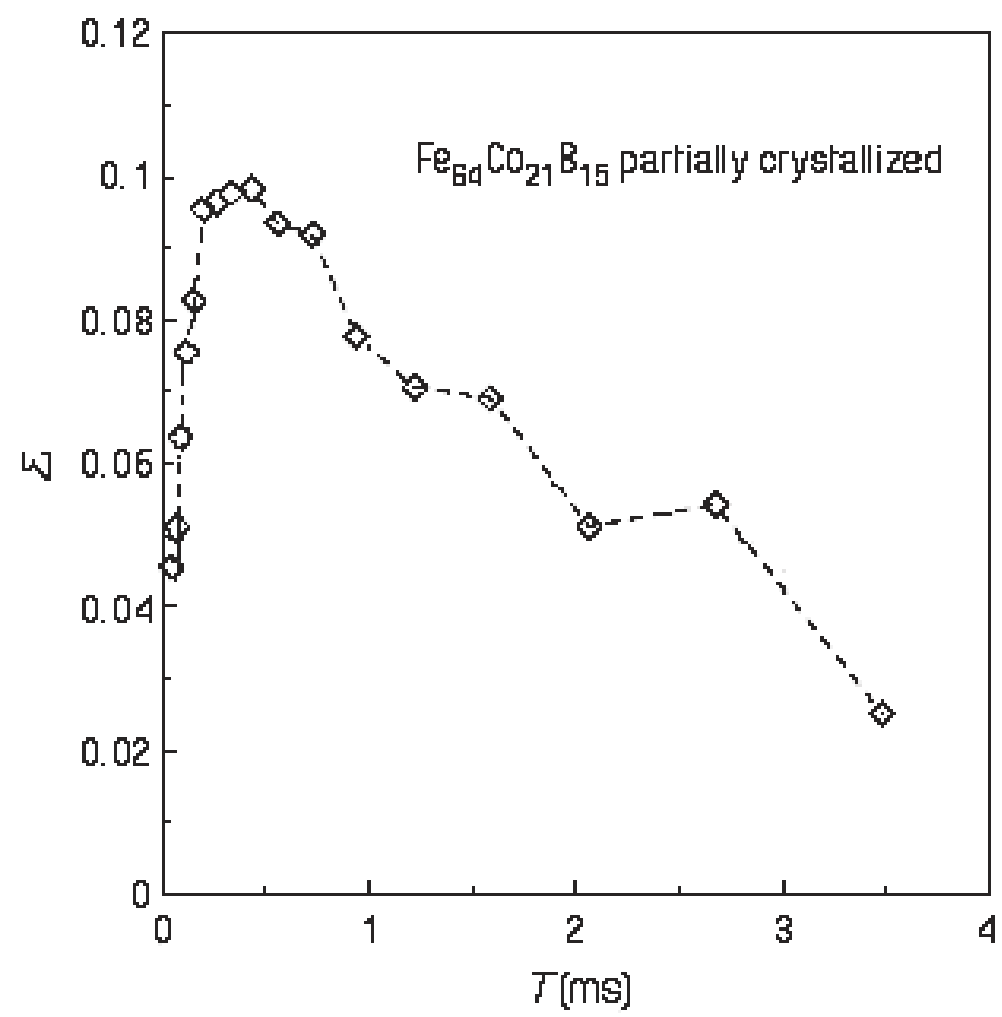

Figure 28. Skewness as a function of the avalanche duration measured in an experiment on a partially crystallized $\mathrm{Fe}_{64} \mathrm{Co}_{21} B_{15}$ sample. (Reprinted from [119]).

equation is overdamped, and neglects inertial effects. Let us mention that the existence of inertial effects in domain wall dynamics, has been demonstrated by Döering [120], who showed that a moving wall differed in energy from the wall at rest by a term due to gyromagnetic effects and proportional to the square wall speed. He identified this as a kinetic energy term and defined the constant of proportionality as one-half of the mass of the wall. The so called Döering mass $M_{D}$ results to be proportional to the inverse wall width divided by the squared gyromagnetic ratio (for a simplified treatment see [121], and also [122] for a review). Inertial effects are therefore always present in domain wall dynamics, however, they are not relevant up to the $\mathrm{GHz}$ band, and can be safely neglected in the equation of motion for the magnetic wall in other regimes, where eddy currents damping largely overwhelms the contribution from Döering mass.

The assumption that the work done by the net field is at any time proportional to the instantaneous velocity is, however, only approximately correct: eddy currents take a finite time to set up after the corresponding wall displacement, and also, they persist for a finite time after the magnetic reversal. 
Because of this delay, the eddy pressure on the wall at a given time $t$ of the avalanche depends on the history of the motion: is not strictly proportional to the instantaneous velocity, but rather it is a weighted average of all the velocities from the beginning of the avalanche up to time $t$.

To go beyond the quasistatic approximation, one has to investigate the dynamical effects eddy current dissipation. Following the lines indicated by [123] this has been done by calculating the magnetic field generated by the eddy currents from the full Maxwell equations [119,124].

Consider a sample with dimension $x \in[-a / 2, a / 2], y \in[-b / 2, b / 2]$, and infinite in the $z$ direction, divided in two magnetic domains by a rigid domain wall on the $y z$ plane, that starts to move from the zero-magnetization position $x=0$, as in figure 29.

The displacement of the magnetic wall in the conducting medium induces a flow of eddy currents that generates a magnetic field $H_{e}$, which, in this geometry, is parallel to the $z$ axis:

$$
\vec{H}_{e}=H_{e}(x, y, t) \hat{z}
$$

Neglecting the displacement currents with respect to the ohmic currents, the Maxwell equation for $H_{e}$ is

$$
\nabla^{2} H_{e}(x, y, t)=\sigma \mu \partial_{t} H_{e}(x, y, t)
$$

where $\sigma$ and $\mu$ are the electric conductivity and the magnetic permeability of the medium respectively.

Equation (97) is a diffusion equation for the eddy field, with a typical timescale for diffusion proportional to $\sigma \mu$. In the quasi-static approximation the permeability is assumed to be negligible within the magnetic domains, and equation (97) reduces to $\nabla^{2} H_{e}=0$, corresponding to an instantaneous eddy field propagation.

This approximation is correct as long as there is a clear separation of timescales, which happens when the observed quantities do not vary appreciably in time on a scale of the order of the characteristic time for diffusion. In that case, one can assume an instantaneous response of the eddy field as given by the static Maxwell equation. However, this is not the case for Barkhausen avalanches, especially for short ones, and therefore the dynamic effect of eddy field has to be taken into account.

Let us go back to equation (97), which has to be solved subject to the appropriate boundary condition

$$
H_{e}=0
$$




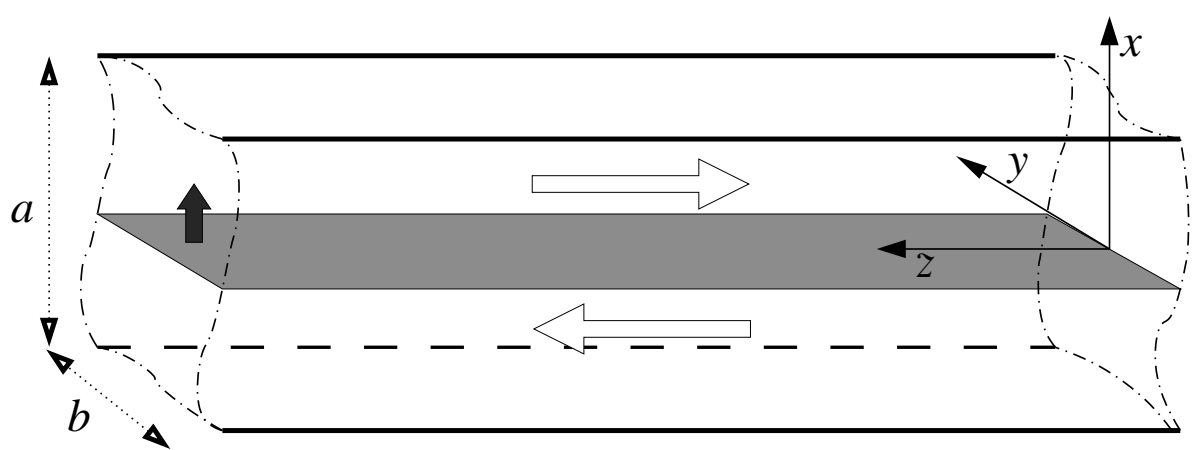

Figure 29. Horizontal arrows indicate the directions of the magnetization in the two domains. The black vertical arrow indicates the direction of motion of the wall.

on the sample surface, and with the Faraday condition

$$
\partial_{x} H_{e}\left(0^{+}, y, t\right)-H_{e} \partial_{x} H_{e}\left(0^{-}, y, t\right)=2 \sigma M_{s} v(t)
$$

that regulates the discontinuity of the field across the wall. In equation $99 M_{s}$ is the saturation magnetization, and $v(t)$ is the instantaneous velocity of the domain wall.

The solution of equation (97) has been derived in [124], by expanding $H_{e}$ in its Fourier components

$$
H_{e}(x, y, t)=\frac{1}{\sqrt{2 \pi}} \int_{-\infty}^{\infty} d \omega F(x, y, \omega) e^{i \omega t}
$$

Each component has to satisfy

$$
\nabla F(x, y, \omega)=r^{2} F(x, y, \omega)
$$

with $r^{2}=i \omega \sigma \mu$. The condition for the magnetic eddy field to be zero on the sample boundary implies that $F(x, \pm b / 2, \omega)=F( \pm a / 2, y, \omega)=0$. The solution of equation (118) for the Fourier components with these boundary condition is of the form

$$
F(x, y, \omega)=\sum_{n=0}^{\infty} A_{n}(x, \omega) \cos \left(\lambda_{n} y\right)
$$

where $A_{n}$ satisfies

$$
\partial_{x}^{2} A_{n}(x, \omega)=\Lambda_{n}^{2} A_{n}(x, \omega),
$$


with $\Lambda_{n}^{2}=\lambda^{2}+r^{2}$, to be solved separately for $x>0$ and $x<0$. The condition on the sample boundary in the $y$ direction implies $\cos \left( \pm b / 2 \lambda_{n}\right)=0$ which fixes $\lambda_{n}=(2 n+1) \pi / b$. The condition on the other boundary in satisfied by choosing

$$
A_{n}(x, \omega)=C_{n}(\omega) \sinh \left(\Lambda_{n}(|x|-a / 2)\right)
$$

so that

$$
F(x, y, \omega)=\sum_{n=0}^{\infty} C_{n}(\omega) \sinh \left(\Lambda_{n}(\omega)(|x|-a / 2)\right) \cos \left(\lambda_{n} y\right)
$$

The functions $C_{n}(\omega)$ are fixed by the Faraday condition around the wall, which in Fourier space reads

$$
\partial_{x} F\left(0^{+}, y, \omega\right)=-\partial_{x} F\left(0^{-}, y, \omega\right)=\sigma M_{s} \hat{v}(\omega)
$$

where $\hat{v}$ is the Fourier transform of the velocity of the wall $v(t)$. Equation (106) implies

$$
\sum_{n=0}^{\infty} C_{n}(\omega) \Lambda_{n}(\omega) \cosh \left(\Lambda_{n}(\omega) a / 2\right) \cos \left(\lambda_{n} y\right)=\sigma M_{s} \hat{v}(\omega)
$$

Multiplying by $\cos \left(\lambda_{m} y\right)$, integrating in $[-b / 2, b / 2]$, and using the orthogonality relations $\int_{-b / 2}^{b / 2} d y \cos \left(\lambda_{n} y\right) \cos \left(\lambda_{m} y\right)=\delta_{n, m} b / 2$, and $\int_{-b / 2}^{b / 2} d y \cos \left(\lambda_{m} y\right)=$ $(-1)^{m} 2 / \lambda_{m}$ one gets

$$
C_{n}(\omega)=(-1)^{n} \frac{4 \sigma M_{s}}{b} \frac{1}{\lambda_{n} \Lambda_{n}(\omega) \cosh \left(\Lambda_{n}(\omega) a / 2\right)} \hat{v}(\omega)
$$

so that the solution for the Fourier components of the field $H_{e}$ is finally found to be given by

$$
F(x, y, \omega)=\frac{4 \sigma M_{s}}{b} \sum_{n=0}^{\infty}(-1)^{n} \frac{\sinh \left(\Lambda_{n}(\omega)(|x|-a / 2)\right)}{\lambda_{n} \Lambda_{n}(\omega) \cosh \left(\Lambda_{n}(\omega) a / 2\right)} \cos \left(\lambda_{n} y\right) \hat{v}(\omega)
$$

To calculate the pressure on the wall due to the presence of eddy currents, 
only the value of the field at $x=0$ is needed, where $F$ simplifies to:

$$
F(0, y, \omega)=\sum_{n=0}^{\infty}(-1)^{n} \frac{4 \sigma M_{s}}{b} \frac{\tanh \left(\Lambda_{n}(\omega) a / 2\right)}{\lambda_{n} \Lambda_{n}(\omega)} \cos \left(\lambda_{n} y\right) \hat{v}(\omega)
$$

The average eddy current pressure on the wall is then obtained by integrating the magnetic field over $y$ at the wall position $x=0$ :

$$
P(t)=\frac{2 I}{b} \int_{-b / 2}^{b / 2} d y H_{e}(0, y, t)=\frac{2 I}{b} \frac{1}{\sqrt{2 \pi}} \int_{-\infty}^{\infty} d \omega e^{i \omega t} \int_{-b / 2}^{b / 2} d y F(0, y, \omega) .
$$

In real space the pressure at time $t$ is given by a convolution of velocities of the wall at all times prior to $t$ with some response function $f$ :

$$
P(t)=\frac{1}{\sqrt{2 \pi}} \int_{-\infty}^{\infty} d s v(t-s) f(s)
$$

which, in Fourier space, corresponds to

$$
\hat{P}(\omega)=-\hat{v}(\omega) \hat{f}(\omega) .
$$

Replacing $F(0, y, \omega)$ in equation (111) with its expression (110), and performing the integral over $y$, we get, for the Fourier transform of the response function, the expression:

$$
\hat{f}(\omega)=\frac{16 I^{2} \sigma}{b^{2}} \sum_{n=0}^{\infty} \frac{\tanh \left(\Lambda_{n}(\omega) a / 2\right)}{\lambda_{n}^{2} \Lambda_{n}(\omega)}
$$

To proceed further it is convenient to simplify the $\omega$ dependence in equation (114), which can be done by using the relation

$$
\tanh (z)=8 z \sum_{k=0}^{\infty} \frac{1}{\pi^{2}(2 k+1)^{2}+4 z^{2}}
$$

with $z=\Lambda_{n}(\omega) a / 2$, which gives

$$
\hat{f}(\omega)=\frac{64 a I^{2} \sigma}{b^{2}} \sum_{n, k=0}^{\infty} \frac{1}{\lambda_{n}^{2}\left(b^{2} \lambda_{k}^{2}+a^{2} \Lambda_{n}^{2}(\omega)\right)} .
$$


Replacing $\lambda_{n}$ and $\Lambda_{n}$ with their expressions, one gets

$$
\hat{f}(\omega)=\frac{64 I^{2} \sigma}{a b^{2} \epsilon^{2}} \overline{\sum_{n, k=1}^{\infty}} \frac{1}{n^{2} \omega_{b}\left(k^{2} \omega_{a}+n^{2} \omega_{b}+i \omega\right)},
$$

where $\bar{\sum}$ indicates a summation over odd numbers only, and $\omega_{a}=\tau_{a}^{-1}=$ $\pi^{2} / \epsilon a^{2}, \omega_{b}=\tau_{b}^{-1}=\pi^{2} / \epsilon b^{2}$.

From equation (117), the expression for the response $f$ in real space is obtained by anti-transforming term to term in the double sum, and replacing each term of the form $1 /\left(\omega_{0}+i \omega\right)$ with its inverse Fourier transform $\sqrt{2 \pi} \exp \left(-\omega_{0} t\right) \theta(t)$ (where $\theta(t)$ is the Heaviside theta function). This leads to:

$$
f(t)=\sqrt{2 \pi} \frac{64 I^{2} \sigma}{a b^{2} \epsilon^{2}} \sum_{n, k=1}^{\infty} \frac{1}{n^{2} \omega_{b}} e^{-\omega_{k, n} t} \theta(t),
$$

where $\omega_{k, n}=\tau_{k, n}^{-1}=k^{2} \omega_{a}+n^{2} \omega_{b}$. The form (118) of the response function gives through the convolution (111) the explicit expression of the retarded pressure on the wall.

The response function finally results to be the sum of simple exponential relaxations, with different relaxation times. The most relevant relaxation time is the largest one, which is

$$
\tau_{0,0}=\frac{\sigma \mu}{\pi^{2}}\left(\frac{1}{a^{2}}+\frac{1}{b^{2}}\right)^{-1} .
$$

This is the quantity that has to be compared with the typical timescale of the phenomena under study to decide whether or not the dynamical effects due to eddy current relaxation are negligible.

In a typical Barkhausen noise experiment, $\tau_{0,0}$ is of the order of $5 \mu \mathrm{s}$. Although this time is small with respect to overall duration of a Barkhausen pulse, the magnetization within an avalanche does vary appreciably on that timescale (see figure 14).

\subsection{Generalized ABBM: negative effective mass}

In terms of the equation of motion, the effect due to the non-instantaneous response of the eddy field to the wall displacement is accounted for by replacing the left hand side of equation (29) with the retarded pressure $P(t)$. This leads 
to a modified $\mathrm{ABBM}$ equation:

$$
\int_{0}^{t} d s f(t-s) v(s)=H(t)-k m+W(m)
$$

that includes the time nonlocal effects.

The eddy dissipation keeps memory of the past velocities of the interface, within a timescale of the order of the eddy field diffusion relaxation time.

To clarify the effects of the response function, it is useful to consider the case of a simple exponential decay $f(t)=\Gamma / \tau_{0} \exp \left(-t / \tau_{0}\right)$, which corresponds to the contribution of a single term in equation (118) to the equation of motion. As long as $\tau_{0}$ is small with respect of the duration of the avalanches considered, only velocities at small times before $t$ will contribute to the convolution, so that we can expand $v(s)$ around $t$ and perform the integrals:

$$
\Gamma \int_{0}^{t} d s \frac{e^{-s / \tau_{0}}}{\tau_{0}} v(t-s) \simeq \Gamma v(t)-\Gamma \tau_{0} v^{\prime}(t)
$$

A simple exponential response function would therefore modify the equation of motion by introducing an inertial term

$$
\Gamma v(t)+M v^{\prime}(t)=H(t)-k m+W(m),
$$

and would lead to the identification of an effective mass $M=-\Gamma \tau_{0}$, which turns out to be negative.

When the full response function is considered, a series of mass and damping terms are generated, each coming from a single exponential. Taking only the leading order in $\mu$, this procedure leads in a similar way to the identification of a damping coefficient

$$
\Gamma=\frac{64 I^{2} \sigma b^{2}}{a \pi^{4}} \Sigma_{1}(b / a)
$$

and of a negative effective mass

$$
M=-\frac{64 I^{2} \sigma^{2} \mu b^{4}}{a \pi^{6}} \Sigma_{2}(b / a),
$$

where $\Sigma_{1}(\alpha)=\bar{\sum}_{n, k} \frac{1}{n^{2}} \frac{1}{n^{2}+\alpha^{2} k^{2}}$, and $\Sigma_{2}(\alpha)=\bar{\sum}_{n, k} \frac{1}{n^{2}} \frac{1}{\left(n^{2}+\alpha^{2} k^{2}\right)^{2}}$, and $\bar{\sum}$ indicates a summation over odd numbers only.

As in the case of the simple exponential relaxation, the ratio between mass 
and damping defines a characteristic time

$$
\tau=|M| / \Gamma=\omega_{b}^{-1} \frac{\Sigma_{2}(b / a)}{\Sigma_{1}(b / a)}
$$

Keeping all orders in $\mu$ results in a frequency dependent effective mass, that has been calculated in [124] and is given by

$$
M=-\frac{64 I^{2} \sigma^{2} \mu b^{4}}{a \pi^{6}} \sum_{n, k=1}^{\infty} \frac{1}{n^{2}\left(\left(k^{2}(a / b)^{2}+n^{2}\right)^{2}+\left(\omega / \omega_{b}\right)^{2}\right)} .
$$

The effective mass turns out to be negative at all frequencies.

The leftward pulse asymmetry observed in Barkhausen experiments is indeed consistent with a negative effective mass: the avalanches start fast and end slowly, which is exactly the opposite of what one would expect from standard inertia. Indeed, since the retarded pressure at time $t$ is given by a weighted average of previous velocities of the wall up to time $t$, this anti-inertial effect can be understood by observing that the effective average velocity is smaller than the instantaneous one for an accelerating wall, while the opposite is true when the wall decelerates.

\subsection{Effective mass dependence on sample geometry}

The role of sample geometry on eddy current retardation has been studied in detail in Ref. [124]. It turns out that the only geometrical parameter that significantly affects the damping coefficient, effective mass, and characteristic time, is the smallest sample dimension.

Figure 30 shows the variation of damping coefficient $\Gamma$, characteristic time $\tau$, and of the modulus of the effective mass $M$, as a function of the aspect ratio $r$ defined as

$$
r=\max (a, b) / \min (a, b),
$$

and keeping constant the section area $A=a b$. These three quantities have a maximum in the case of a square rod. Therefore this is the geometry where, $A$ being constant, the maximum asymmetry is expected to be observed.

Figure 31 shows the variation of the the same three quantities as a function of the most relevant parameter, which is the smallest sample dimension $d=$ $\min (a, b)$. The damping coefficient $\Gamma$, characteristic time $\tau$, and modulus of the effective mass $M$, increase as $d, d^{2}$, and $d^{3}$ respectively, and saturate to the values $A^{1 / 2}, A$, and $A^{3 / 2}$, respectively. The role of the two dimensions $a$, 


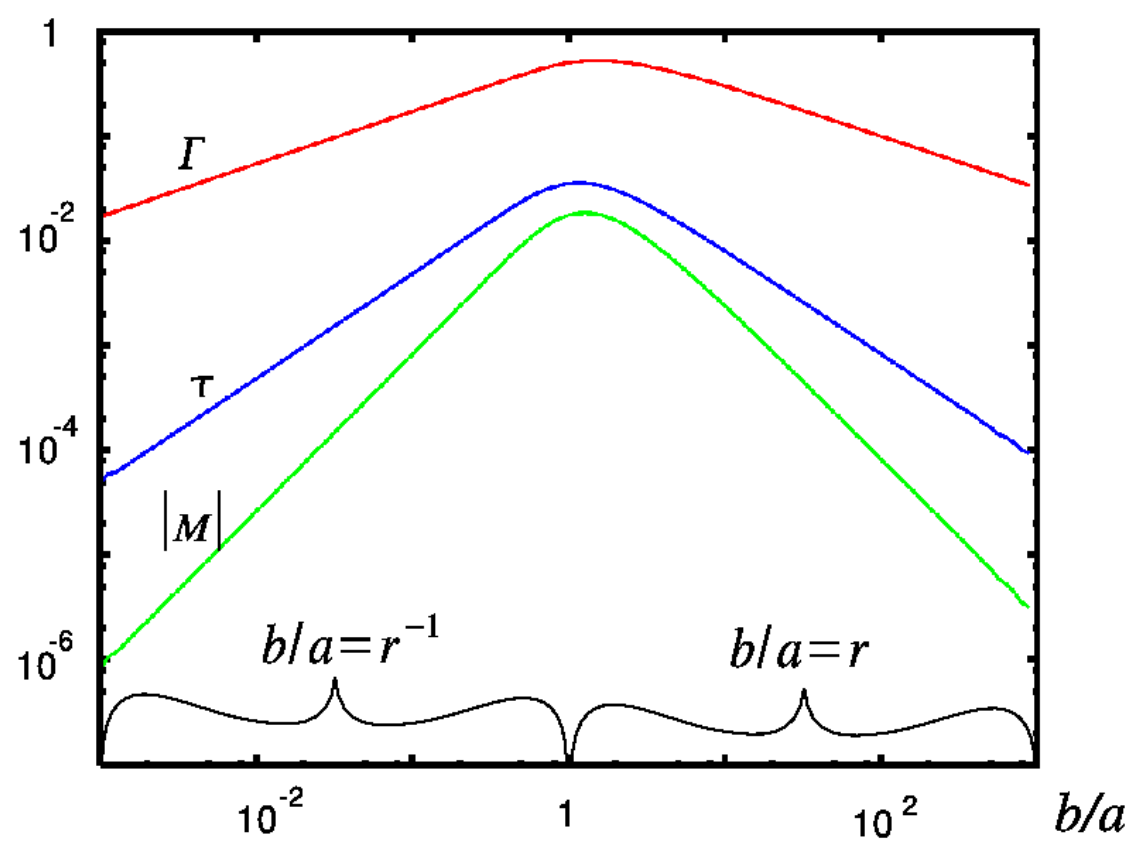

Figure 30. Damping coefficient $\Gamma$, characteristic time $\tau$, and modulus of the effective mass $M$, as functions of the aspect ratio $r$.

and $b$, transverse and parallel to the wall is however not symmetric, and the effect of eddy current retardation is expected to be more severe, other things being equal, when the transverse dimension is the smallest.

The analytical expressions of $\Gamma, \tau$, and $M$ used in figures 30, and 31 are given in [124].

It would be interesting to repeat $\mathrm{BN}$ experiments in changing sample geometries in order to test these results.

Let us comment on the relevance of the eddy inertial term when compared to the usual inertial term which is associated to the Döring mass $M_{D}$. The values of $M_{D}$ are about four orders of magnitude smaller then the eddy mass $M$ in typical sample geometries used in BN experiments. Indeed the Döring mass originates from a gyroscopic precessional effect, whose associated time scales are much faster then those relative to the eddy mass. However, the analysis reported in [124] on the dependence on sample geometry predicts a fast decay $d^{-3}$ of the mass with the sample thickness, which means that the two masses may become comparable in thick films. 




Figure 31. Damping coefficient $\Gamma$, the characteristic time $\tau$, and the modulus of the effective mass $M$, as functions of the smaller sample dimension $d$, both in the two cases, when $d$ equals the dimension transverse $(a)$ and parallel $(b)$ to the domain wall.

\subsection{Comparison between theory and experiments}

By simulating numerically the modified ABBM equation (120) one observes that, whereas the distributions of avalanche duration and size are unaffected by the addition of the inertial term, the pulse shapes become asymmetric and bear a remarkable similarity with the experimental ones (see figures 32,33 , where the curves are normalized with $\left.N=\int_{0}^{T} d t\langle v(t, T)\rangle / T\right)[119]$. Note that eddy currents only change the internal dynamics of the avalanches, so that at least the distribution of avalanches sizes' is expected to be left unaltered, while correction could be present in the power spectrum (see below) and in the distribution of avalanche durations' (not observed).

The skewness of the simulated model results positive, indicating a leftward asymmetry, corresponding to a negative mass, while it would be negative, indicating rightward asymmetry, in the standard case of a positive mass. The qualitative behavior of the skewness as a function of the avalanche duration in correspondence to a negative mass (see figure 34) is similar to the one observed in the experiments (see figure 28): it is always positive, it increases from zero, shows a peak at some characteristic time, and it decays to zero for long avalanches. The existence of a peak can be used to extract the characteristic 


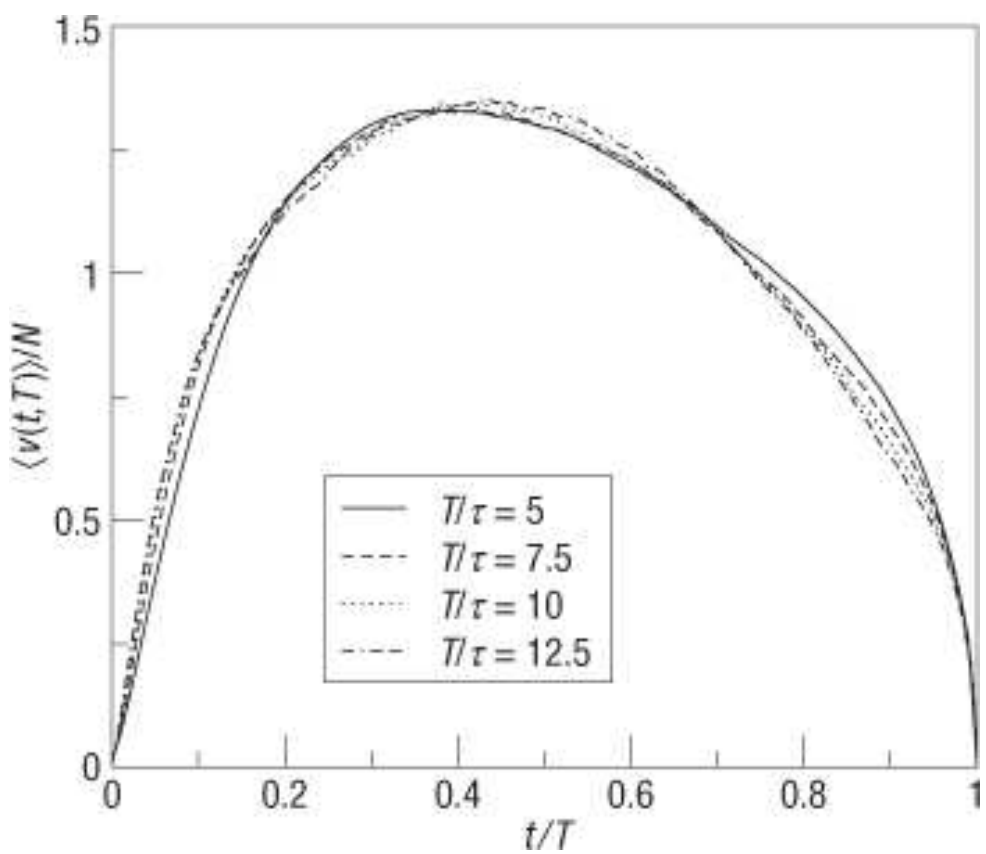

Figure 32. Asymmetry of the rescaled pulse shape: pulses obtained from Barkhausen noise experiments in a partially crystallized $\mathrm{Fe}_{64} \mathrm{Co}_{21} \mathrm{~B}_{15}$ ribbon. The shapes for different durations $T$. (Reprinted from [119]).

relaxation timescale, which corresponds to the ratio between mass and damping constant, and to make a quantitative comparison between the model and experiments.

A further quantitative test of the theory against experiments includes the comparison of the location of the peak $T_{P}$ that characterizes the skewness versus duration plot. Skewness extracted from experimental pulses shows a $T_{p}$ at a value which is roughly twice that obtained from simulations of the modified $\mathrm{ABBM}$ equation with the microscopic parameters of the corresponding experiment. This may be due to the fact that the model considers just one single domain wall, while many domains may be present in general in a sample. It is indeed expected [123] that the relaxation time increases with the number of domains.

Another interesting point to underline is the effect of the negative inertial term on the power spectra. Power spectra obtained by simulating the modified $\mathrm{ABBM}$ equation show a deviation from the large frequency asymptotic regime at a frequency $\omega^{*}=T_{P}^{-1}$ very similar to the one observed in some experiments. Moreover, experimental data also show a correspondence between the peak in the skewness and the frequency at which the deviation appears in the spectrum [118].

Summarizing the discussion of this section, we can conclude that the left- 


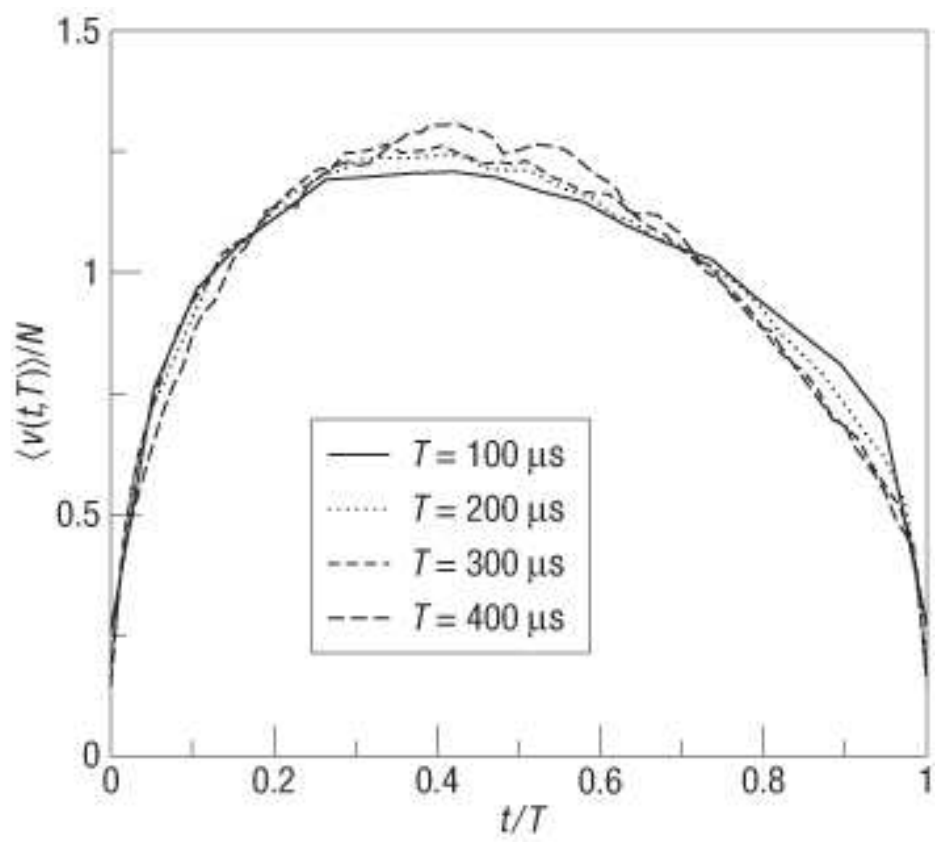

Figure 33. The shapes obtained from the modified ABBM model with the parameters corresponding to those of the experiment in figure 32 (Reprinted from [119]).

ward asymmetry of pulse profiles in $\mathrm{BN}$ is caused by the non instantaneous response of the eddy field to the wall displacement, that can be accounted for by associating a negative effective mass to the moving wall. The asymmetry is a non-universal property, that depends on the material parameters $\sigma, \mu$, and $M_{s}$ and on the geometrical dimensions $a$ and $b$ of the sample, and therefore it allows to extract information about the characteristic time ruling the microscopic dynamics.

Since the asymmetry is related to the existence of this microscopic timescale, its magnitude depends on the avalanche duration: for avalanches long enough with respect to the characteristic time, the microscopic details of the material and the geometry of the sample become irrelevant, pulse-shapes become symmetric, and universality is expected to hold.

Let us finally comment on the physical meaning of the eddy inertial term. It has always to be kept in mind that the negative effective mass is just a convenient way to account, to the first order, for the history dependent damping. The effective mass, however, is unable to describe for example the very short time dynamics, when the domain wall starts to move from its position at rest. Indeed, in this case, the negative mass would cause the wall to move backward, which is certainly not the case. Here the results of this section should be used with more care: when the wall starts to move there is no previous motion his- 


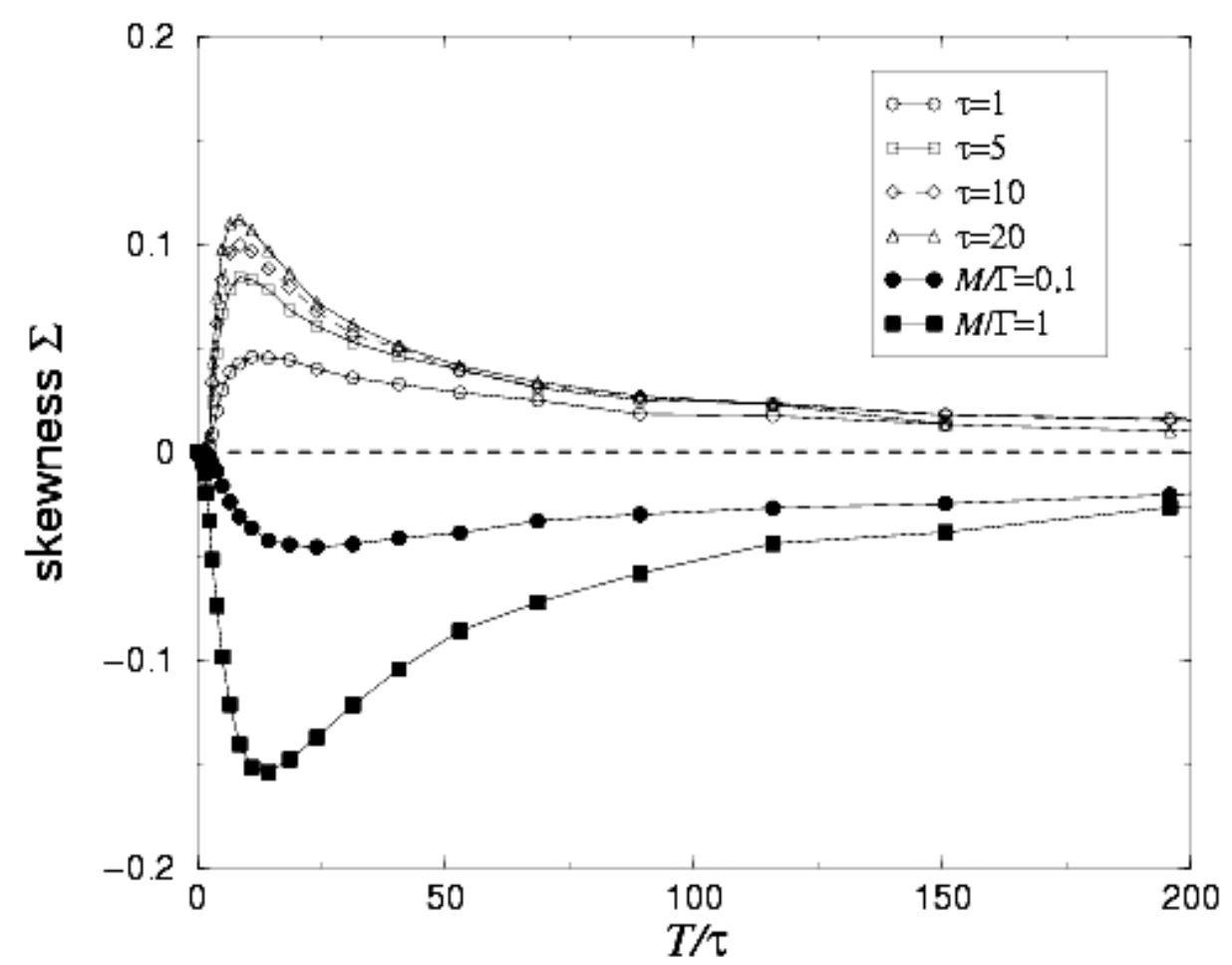

Figure 34. Skewness from simulations of the modified ABBM model. Filled symbols correspond to positive mass (negative skewness), empty symbols to negative mass (positive skewness). (Reprinted from [119]).

tory, and therefore both the eddy damping and the eddy inertial term are zero. In the very short time dynamics the Döring mass, which is positive, represents the most relevant inertial term in the equation of motion. Therefore standard inertia governs the dynamics, until damping and eddy mass build up.

6 Other crackling systems: granular materials, seismic activity in earthquakes

Crackling noise emerges in very different contexts, and the similarity in how completely different systems behave suggests the existence of some common principle in the underlying physics. As an example of how ideas developed in the study of one crackling system can stimulate advances in other fields, we report here some analogies between $\mathrm{BN}$ and two other crackling system: sheared granular materials and seismic activity in earthquakes. 
(a)

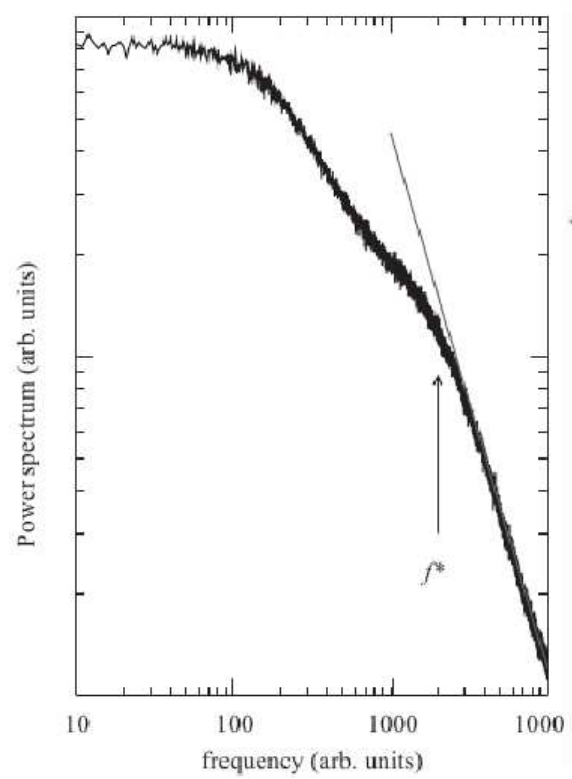

(b)

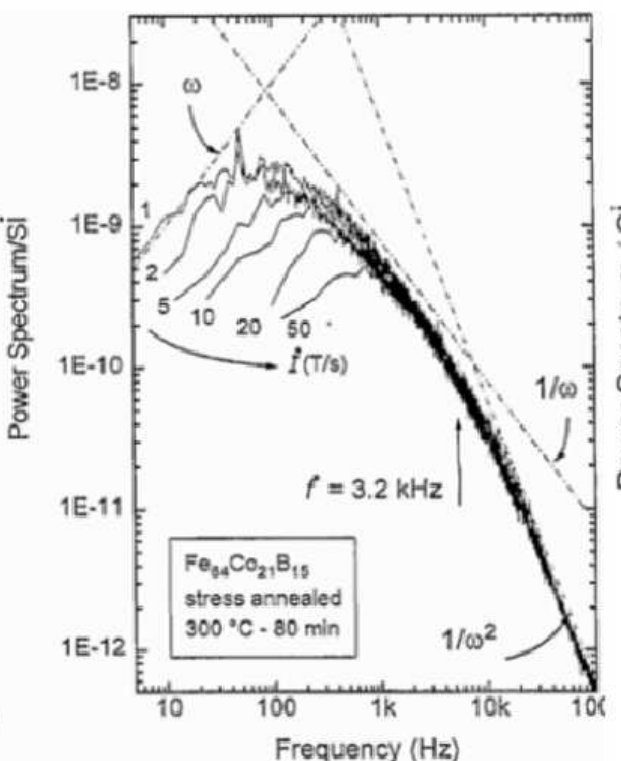

(c)



Figure 35. Comparison of power spectra where deviation from $1 / \omega^{2}$ is observed at intermediate frequencies with the one from simulations. (a) Power spectrum from a simulation of the modified ABBM model. (Reprinted with permission from [118]). [118] Copyright 2007 by Elsevier. (b) Power spectrum for a partially crystallized $\mathrm{Fe}_{64} \mathrm{Co}_{21}$ B15.(Reprinted with permission from [81]). 81] Copyright 1996 by Elsevier. (c) Power spectrum for a polycrystalline 7.8\% FeSi. (Reprinted with permission from [58]). [58] Copyright 1997 by World Scientific, Singapore. 


\subsection{Dynamics of granular materials under shear}

A granular medium, subject to a slow loading rate, has an intermittent shear response characterized by large fluctuations, typical of crackling systems $[125$, 126]. The large fluctuations in this case originate from the rearrangement of the network of force chains along which the stress propagate.

Most of the past theoretical activity on granular matter has focused on average properties, that could allow the formulation of macroscopic laws governing the dynamics. However, more recently it became clear that a deeper understanding of the mechanisms that regulate the dynamics on smaller scales and cause the large fluctuations, cannot be reached without going through a detailed analysis of the slip statistics.

In this framework, a very interesting model has recently been proposed to interpret a series of experiments on granular materials sheared in a Couette geometry [7]. This model, is based on an assumption similar to the one that underlies the ABBM model for BN, namely that the resultant from the forces acting on the system performs a Brownian motion.

In the experiment a cylindrical cell is filled with monodisperse glass beads with $2 \mathrm{~mm}$ of diameter. The shear is created by rotating an annular plate over the top of the circular channel, driven by a torsion spring with stiffness $k$. After being initialized to reach the stationary state, the system is then run at a slow driving velocity $v$. The experimental device allows to measure the angular position $\theta$ of the plate, and the deflection of the torsion spring. In a given range of driving velocities, the system displays a stick-slip regime, where repeatedly the torque on the plate accumulates as the spring winds up, until the plate slips.

The instantaneous velocity of the plate $\dot{\theta}$ as a function of time, shows the typical intermittent behavior of crackling signals, with pulses of largely fluctuating amplitudes. A statistical analysis of the signal similar to the one usually done for BN emissions reveals the presence of power law distributions of the slip events in the stick-slip phase, as it was already observed in a previous experiment [126].

In this case the pulse size $s$ corresponds to the angular variation during the slip event. The sizes and durations distributions show a more complex shape than in the BN case, since the initial power law decay is followed by a peak at larger scales, in correspondence to some cut-off value. The position of the peak is independent of the driving velocity $v$.

As in the case of $\mathrm{BN}$, the intermittent stick-slip phase is observed only as long as the driving velocity is not too large. Above a threshold value, a transition to a steady sliding regime occurs, that is analogous to the transition between the intermittent $\mathrm{BN}$ noise regime, and the unique avalanche magnetization reversal that occurs at high sweep field rates. 
In ref. [7] the authors propose a simple model that quantitatively reproduces the phenomenology, and which is based on some minimal hypothesis. The equation of motion for the stick-slipping plate is obtained by equating the inertial term to the driving force on the spring diminished by the counteracting friction of the granular media.

$$
I \ddot{\theta}=k(v t-\theta)-F .
$$

Here $I$ is the moment of inertia of the system, and the whole complexity of the granular matter dynamics that give rise to the wide fluctuations is accounted for in the frictional torque term $F$.

Baldassarri et al. assume that, as a first approximation, the instantaneous frictional torque is a function of $\theta$ and $\dot{\theta}$ only (since $F$ is constant when the disk is stuck, there is no direct dependence on time $t$ in $F$ ), and that $F$ can be split into a deterministic part $F_{d}(\dot{\theta})$ plus a random contribution $F_{r}(\theta)$.

The deterministic part of the instantaneous friction $F_{d}(\dot{\theta})$ is obtained by fitting the experimental average velocity dependence of the frictional torque with the expression

$$
F_{d}(\dot{\theta})=F_{0}+\gamma\left(\dot{\theta}-2 v_{0} \ln \left(1+\dot{\theta} / v_{0}\right)\right)
$$

where $F_{0}$ is the average static friction force, $v_{0}$ is the minimum average torque and $\gamma$ is the high velocity damping. Note, that the results obtained, however, do not depend very much on the precise choice of the function $F_{d}$.

The fluctuating part of the instantaneous friction is the one that accounts for the continuously rearranging disordered structure of the force chain network present in the granular medium. This is assumed to be described by a confined Brownian process

$$
\frac{d F_{r}(\theta)}{d \theta}=\eta(\theta)-a F_{r}(\theta)
$$

where $\eta$ is a Gaussian quenched uncorrelated noise, and $a$ is an inverse correlation length. The idea is that the frictional torque changes of a random amount after every small slip, but eventually decorrelate on large slips involving a complete rearrangement of the grains, resulting in a confinement of the Brownian process. The quantity $a^{-1}$ plays the same role as the correlation length of the pinning field $\xi_{P}$ introduced in the ABBM model (see section 4.8).

This theory shows a number of analogies with the ABBM theory for BN. The granular matter dynamics is more complicated than the one of magnetic domains, due to the presence of inertial effects and of a shear rate weakening torque. However, both theories are based on the same assumption that the 
effective force acting on the system performs a Brownian motion. Indeed, the presence of Brownian correlations in the effective force evolution, due to collective effects (the sum over many pinning forces over the domain wall in one case, the formation and destruction of force chains in the granular media in the other) may be of more general validity in driven dissipative systems.

The equation of motion is also similar in the two cases, and, although, the one describing the granular material includes more complex effects, it reduces to the $\mathrm{ABBM}$ equation in the limit $I, v_{0} \rightarrow 0$ of vanishing inertia and minimal average torque. In this case, or in an appropriate velocity regime $v_{0} \ll v \ll \gamma / I$, where the additional effects can be neglected, all the results obtained for the ABBM model are valid, once magnetization is interpreted as a slip, the magnetic field rate as a shear rate, the demagnetizing factor as the stiffness of the torsional spring, the eddy current damping as a frictional damping, and the random pinning as a random friction.

The analogy also suggests that the transition from the stick-slip phase at slow drivings to the sliding phase at fast drivings might possibly be explained also in this case as a transition from transient to recurrent for the corresponding stochastic process.

\subsection{Seismic activity in earthquakes}

Another kind of crackling noise that shows analogies with $\mathrm{BN}$ is the seismic activity during earthquakes.

Earthquake phenomenology exhibits a number of power law distributions including the Gutenberg-Richter frequency-size statistics $[127,128]$ and the Omori law for aftershock decay rates $[129,130]$.

In literature, the area of the region of the fault that slips in an earthquake is called the moment. The probability of occurrence of an earthquake of a given moment $M$ decays as a power law according to

$$
P(M) \sim M^{-(1-\beta)}
$$

The exponent $\beta$ is the same, within errors, for most sets of data, collected in different times and regions.

As in the case of BN, scaling functions have been introduced as additional interesting observables, which provides a powerful tool both to check universality, and to test the theory against data. In particular, the average moment rate profiles at fixed total earthquake duration, have been studied in [30], and compared with the prediction of a mean field theory for earthquakes dynamics [131]. 




Figure 36. A collapse of averaged earthquake pulses' shapes, with a duration of $T$, with two to ten earthquakes averaged for each value of $T$. The collapse was obtained using the mean field theory for which the universal scaling function is claimed to be $g(x)=A x(1-x)$ with $x=t / T$ [51]. Note the apparent asymmetry to the left in the observed data while the theoretical curve is symmetric around its maximum. In the inset the raw data and the averaged data before collapse are shown.

(Reprinted with permission from [30]). [30] Copyright 2006 by the American Physical Society.

The average moment rate is supposed to behave as

$$
\langle d M(t, T) / d t\rangle \simeq T g(t / T) .
$$

In paper [30] the universal function $g(t / T)$ is claimed to be:

$$
g(x)=A(x(1-x)) .
$$

This result was obtained by mapping the model onto the mean field model for $\mathrm{BN}$, for which the result of $g$ being an inverted parabola was claimed in [51].

It is clear from figure 36 that in seismic data the moment rate of a given duration $T$ increases faster then it drops, as the corresponding average moment 
shapes show a leftward asymmetry similar to that observed in BN, while, also in this case, models predict a symmetric shape.

Seismic movements are caused by the motion of fault planes in response to the accumulated stress. The analogy with BN suggests the possibility that the origin of the asymmetry is similar in the two cases. The asymmetry may indeed originate from some non-local dynamical effect, that, in the case of earthquakes could be caused by the presence of stress overshoots [132]. It would be interesting to see how the skewness of the average pulses changes with the duration of the seismic event (which is not clear for the data in ref. [71]), as this can help to understand whether or not also in this case the asymmetry disappears in large events, and whether universality is recovered oon large time scales.

In the case of earthquakes, the analogy with domain wall dynamics is even more stringent than in the case of sheared granular media. Indeed, the mean field description studied in [30] exactly coincides with the ABBM model. Long range elastic interactions lead to $d=3$ being the upper critical dimension for earthquakes models $[93,131,133,134]$, and playing the same role as the long range dipolar interactions in $\mathrm{BN}$.

It is interesting to mention that asymmetric avalanches are also observed in other crackling systems. The dynamics of the Bak-Tang-Wiesenfeld model of sand piles [135] is characterized by asymmetric avalanches [52]. Leftward asymmetric pulses also appear in the intermittent behavior of systems undergoing a shear deformation that proceeds in bursts of dislocations activity [136]. Recently Laurson and Alava [137] analyzed a discrete dislocation dynamics model and observed, in agreement with the experiments, a leftward asymmetry in the avalanche shape, corresponding to an asymmetry in the average creation and annihilation rates of dislocations during the avalanche. They also find that the asymmetry decreases with the signal threshold, although the threshold value do not have visible effects on the avalanche statistics. Finally, a strong skewness in the avalanche shape has recently been observed in the acustic emissions during martensitic transformations [138].

\section{Conclusions}

In this review we report on the present state of understanding of the Barkhausen effect in soft ferromagnetic materials, focusing on those with long range dipolar interactions. In particular, we collect several results on the mean field ABBM model for BN. This model, originally proposed on phenomenological basis, corresponds to a mean field description for an elastic magnetic wall in a disordered ferromagnet under the effect of an external driving field, and is extremely successful in reproducing the complex phenomenology observed 
in the experiments, at least as long as universal properties are involved.

The ABBM model consists of an effective equation of motion for the velocity of the avalanche front. This equation maps onto the Langevin equation for a biased random walk in a logarithmic potential with appropriate boundary conditions. This process is amenable to analytical treatment with standard methods in the theory of stochastic processes. Therefore this mapping has the advantage to provide several exact results, as well as a direct interpretation of a number of properties observed in the experiments in terms of properties of the corresponding stochastic process. Specifically, through this mapping, the power laws in the distributions of sizes and durations of avalanches are exactly derived in terms of distributions of return times to the origin, the continuous dependence of the exponents in the power law distributions on the driving field rate can be calculated and turns out to be related to the marginality of the logarithmic perturbation to a free random walk, the existence of a threshold in the driving field rate has a clear interpretation (thanks to a further mapping) in terms of recurrence properties of a free random walk, the existence of a cutoff in the power law distributions is ascribed to the bias term, and therefore is due to the presence of the demagnetizing field, and finally the average shape of the Barkhausen pulse which is related to the excursion of the process, and the power spectra of the noise can be computed explicitly.

Although deviations from the ABBM predictions are observed in some experiments, the picture that emerges from this theory is quite satisfactory. The most relevant experimental feature that is not reproduced by ABBM is the leftward asymmetric shape systematically observed in experiments. This asymmetry is due to a non-local damping by the eddy currents, whose effect is neglected in the original ABBM model formulation, as it is in most domain walls models. This effect can be taken into account by including in the equation of motion an inertial term with a negative effective mass for the interface, which leads to the definition of a generalized ABBM model. The asymmetry of the pulses depend on the duration and can be used to extract important information on the characteristic time of the underlying dynamics.

A basic point to keep in mind, is that the whole theory developed so far models one single domain wall, while many interacting domain walls are in general involved in the magnetization process. It would therefore be interesting to understand how the presence of many domains modifies the present theory.

Although the ABBM theory was derived as a model for Barkhausen noise, it turns out to be a paradigmatic theory for avalanche dynamics, and can give suggestions on how to model other crackling systems. We discussed some analogies with sheared granular matter and seismic events. These similarities point to the existence of deep connections between the physics of avalanches in different systems. Also the presence of non-universal effects might be common to other crackling system. Asymmetric avalanche shapes similar to those 
observed in BN appear in other crackling systems: in martensitic transformations, in plastic deformations, in the Bak-Tang-Wiesenfeld model of sand piles, and in seismic events. In the last case, the leftward asymmetric average moments may originate, from the presence of stress overshoots.

\section{Acknowledgments}

The author wishes to thank all those people who have contributed to our current understanding of the problems discussed. In particular, the personal author's understanding of these subjects is largely due to interactions with A. Baldassarri, G. Bertotti, C. Castellano, F. Dalton, G. Durin, A. Petri, J. P. Sethna, and S. Zapperi, to whom the author is most grateful also for stimulating discussions and for encouragement to write this review.

\section{References}

[1] J.P. Sethna, K.A. Dahmen, and C.R. Myers, Crackling noise, Nature 410 (2001), pp. 242-250.

[2] J.P. Sethna, 2007, Vol. 85, 6. in Complex Systems, Volume LXXXV: Lecture Notes of the Les Houches Summer School 2006 Elsevier, Amsterdam ed. by Jean-Philippe Bouchaud, Marc Mezard, and Jean Dalibard, pp. 257-288.

[3] H. Barkhausen, Zwei mit hilfe der neuen verstãrker entdeckte erscheinugen, Physik Z 20 (1919), pp. 401-403.

[4] G. Bertotti Hysteresis in Magnetism: For Physicists, Material Scientists, and Engineers, Academic Press, San Diego, 1998.

[5] G. Durin and S. Zapperi, 2006, Vol. 2in The Science of Hysteresis Elsevier, Amsterdam ed. by G. Bertotti and I. D. Mayergoyz, pp. 181-267.

[6] A. Baldassarri et al., 2007in Traffic and Granular Flow'05 Springer, Berlin, pp. 91-100.

[7] A. Baldassarri et al., Brownian Forces in Sheared Granular Matter, Physical Review Letters 96 (2006), pp. 118002-4.

[8] M. Bretz et al., Broad distribution of stick-slip events in Slowly Sheared Granular Media: Table-top production of a Gutenberg-Richter-like distribution, Europhysics Letters 74 (2006), pp. 1116-1122.

[9] E. Vives et al., Distributions of avalanches in martensitic transformations, Physical Review Letters 72 (1994), p. 1694.

[10] M.C. Miguel et al., Intermittent dislocation flow in viscoplastic deformation, Nature 410 (2001), pp. 667-671.

[11] J. Weiss and D. Marsan, Three-Dimensional Mapping of Dislocation Avalanches: Clustering and Space/Time Coupling, Science 299 (2003), pp. 89 - 92.

[12] M. Zaiser, Scale invariance in plastic flow of crystalline solids, Advances in Physics 55 (2006), pp. $185-245$.

[13] F.F. Csikor et al., Dislocation Avalanches, Strain Bursts, and the Problem of Plastic Forming at the Micrometer Scale, Science 318 (2007), pp. 251-254.

[14] J.P. Sethna, Crackling wires, Science 318 (2007), pp. 207-208.

[15] D. Ertaş and M. Kardar, Anisotropic Scaling in Depinning of a Flux Line, Physical Review Letters 73 (1994), p. 1703.

[16] S. Field et al., Superconducting Vortex Avalanches, Physical Review Letters 74 (1995), p. 1206.

[17] D. Ertaş and M. Kardar, Anisotropic scaling in threshold critical dynamics of driven directed lines, Physical Review B 53 (1996), p. 3520.

[18] R.A. Guyer and K.R. McCall, Capillary condensation, invasion percolation, hysteresis, and discrete memory, Physical Review B 54 (1996), p. 18.

[19] M.P. Lilly, A.H. Wootters, and R.B. Hallock, Spatially Extended Avalanches in a Hysteretic 
Capillary Condensation System: Superfluid 4He in Nuclepore, Physical Review Letters 77 (1996), p. 4222.

[20] P. Bak, M. Paczuski, and M. Shubik, Price variations in a stock market with many agents, Physica A 246 (1997), pp. 430-453.

[21] J. Bouchaud, Power-laws in economics and finance: some ideas from physics, Quantitative Finance 1 (2001), pp. 105-112.

[22] J.P. Bouchaud, The subtle nature of financial random walks, Chaos: An Interdisciplinary Journal of Nonlinear Science 15 (2005), pp. 026104-10.

[23] E.V. Colla, L.K. Chao, and M.B. Weissman, Barkhausen Noise in a Relaxor Ferroelectric, Physical Review Letters 88 (2001), p. 017601.

[24] W.A. Curtin and H. Scher, Analytic model for scaling of breakdown, Physical Review Letters 67 (1991), p. 2457.

[25] A. Petri et al., Experimental Evidence for Critical Dynamics in Microfracturing Processes, Physical Review Letters 73 (1994), p. 3423.

[26] S. Zapperi, A. Vespignani, and H.E. Stanley, Plasticity and avalanche behaviour in microfracturing phenomena, Nature 388 (1997), pp. 658-660.

[27] A. Garcimartín et al., Statistical Properties of Fracture Precursors, Physical Review Letters 79 (1997), p. 3202.

[28] L.I. Salminen, A.I. Tolvanen, and M. Alava, Acoustic Emission from Paper Fracture, Physical Review Letters 89 (2002), p. 185503.

[29] I. Main, Statistical Physics, Seismogenesis, and Seismic Hazard, Reviews of Geophysics 34 (1996), pp. 433-462.

[30] A.P. Mehta, K.A. Dahmen, and Y. Ben-Zion, Universal mean moment rate profiles of earthquake ruptures, Physical Review E 73 (2006), pp. 056104-8.

[31] B. Alessandro et al., Domain-wall dynamics and Barkhausen effect in metallic ferromagnetic materials. I. Theory, Journal of Applied Physics 68 (1990), pp. 2901-2907.

[32] B. Alessandro et al., Domain-wall dynamics and Barkhausen effect in metallic ferromagnetic materials. II. Experiments, Journal of Applied Physics 68 (1990), pp. 2908-2915.

[33] A.J. Bray, Random walks in logarithmic and power-law potentials, nonuniversal persistence, and vortex dynamics in the two-dimensional XY model, Physical Review E 62 (2000), p. 103.

[34] P. Weiss, L'hypothèse du champ moléculaire et la propriété ferromagnétique, J. de Physique 6 (1907), pp. 661-690

[35] W.C. Elmore, The Magnetic Structure of Cobalt, Physical Review 53 (1938), p. 757.

[36] H.J. Williams and W. Shockley, A Simple Domain Structure in an Iron Crystal Showing a Direct Correlation with the Magnetization, Physical Review 75 (1949), p. 178.

[37] C. Kittel, Physical Theory of Ferromagnetic Domains, review of modern physics 21 (1949).

[38] K. Tiitto, Use of Barkhausen noise in fatigue - Nondestructive Testing and Evaluation, Nondestructive Testing and Evaluation 5 (1989), pp. 27-37.

[39] M.R. Govindaraju et al., Evaluation of fatigue damage in steel structural components by magnetoelastic Barkhausen signal analysis, in 37 th Annual conference on magnetism and magnetic materials, Vol. 73 AIP, Houston, Texas (USA), 1993, pp. 6165-6167.

[40] L.B. Sipahi, Overview of applications of micromagnetic Barkhausen emissions as noninvasive material characterization technique, in 38th Annual Conference on Magnetism and Magnetic Materials, Vol. 75 AIP, Minneapolis, Minnesota (USA), 1994, pp. 6978-6980.

[41] C.C.H. Lo et al., Monitoring fatigue damage in materials using magnetic measurement techniques, J. Appl. Phys. 85 (1999), pp. 4595-4597.

[42] B. R et al., Characterisation of Microstructures, Deformation, and Fatigue Damage in Different Steels Using Magnetic Barkhausen Emission Technique, Russian Journal of Nondestructive Testing 37 (2001), pp. 789-798.

[43] R. B et al., Assessment of microstructures and mechanical behaviour of metallic materials through non-destructive characterisation, International Materials Reviews 48 (2003), pp. 273325.

[44] S.P. Sagar et al., Magnetic Barkhausen emission to evaluate fatigue damage in a low carbon structural steel, International Journal of Fatigue 27 (2005), pp. 317-322.

[45] V. Moorthy, B. Shaw, and P. Hopkins, Magnetic Barkhausen emission technique for detecting the overstressing during bending fatigue in case-carburised En36 steel, NDT \& E International 38 (2005), pp. 159-166.

[46] R.C. O'Handley Modern Magnetic Materials: Principles and Applications, John Wiley \& Sons, New York, 2000.

[47] G. Bertotti, F. Fiorillo, and M.P. Sassi, Barkhausen noise and domain structure dynamics 
in Si-Fe at different points of the magnetization curve, Journal of Magnetism and Magnetic Materials 23 (1981), pp. 136-148.

[48] G. Durin and S. Zapperi, The role of stationarity in magnetic crackling noise, Journal of Statistical Mechanics 2006 (2006), p. P01002.

[49] D. Spasojević et al., Barkhausen noise: Elementary signals, power laws, and scaling relations, Physical Review E 54 (1996), p. 2531.

[50] D. Sornette, Sweeping of an instability : an alternative to self-organized criticality to get powerlaws without parameter tuning, J. Phys. I France 4 (1994), pp. 209-221.

[51] M.C. Kuntz and J.P. Sethna, Noise in disordered systems: The power spectrum and dynamic exponents in avalanche models, Physical Review B 62 (2000), p. 11699.

[52] L. Laurson, M.J. Alava, and S. Zapperi, Power spectra of self-organized critical sandpiles, Journal of Statistical Mechanics (2005), p. L11001.

[53] G. Durin, G. Bertotti, and A. Magni, Fractals, scaling and the question of self-organized criticality in magnetization processes, Fractals 3 (1995), pp. 351-370.

[54] G. Durin,, and S. Zapperi, Scaling Exponents for Barkhausen Avalanches in Polycrystalline and Amorphous Ferromagnets, Physical Review Letters 84 (2000), p. 4705.

[55] R.A. White and K.A. Dahmen, Driving Rate Effects on Crackling Noise, Physical Review Letters 91 (2003), p. 085702.

[56] S. Zapperi et al., Dynamics of a ferromagnetic domain wall: Avalanches, depinning transition, and the Barkhausen effect, Physical Review B 58 (1998), p. 6353.

[57] G. Durin and S. Zapperi, Universality and size effects in the Barkhausen noise, J. Appl. Phys. 87 (2000), pp. 7031-7033.

[58] G. Durin, The Barkhausen effect: new perspectives for an old problem, in Proc. of the 14th Internation Conference on Noise in Physical Systems and $1 / f$ FluctuationsNoise as a Tool for Studying Materials. Ed. C. Claeys and E. Simoen World Scientific, Singapore, 1997, pp. $577-582$.

[59] G. Coudercon et al., Magnetization process in NiFe alloys with vanishing anisotropies, Magnetics, IEEE Transactions on 25 (1989), p. 3973

[60] G. Bertotti, F. Fiorillo, and A. Rietto, Microscopic and macroscopic aspects of the magnetization process in SiFe single crystals, Magnetics, IEEE Transactions on 20 (1984), p. 1481.

[61] K.P. O'Brien and M.B. Weissman, Statistical characterization of Barkhausen noise, Physical Review E 50 (1994), p. 3446.

[62] J.R. Petta, M.B. Weissman, and G. Durin, Barkhausen pulse structure in an amorphous ferromagnet: Characterization by high-order spectra, Physical Review E 57 (1998), p. 6363.

[63] J.P. Sethna et al., Hysteresis and hierarchies: Dynamics of disorder-driven first-order phase transformations, Physical Review Letters 70 (1993), p. 3347.

[64] K. Dahmen and J.P. Sethna, Hysteresis loop critical exponents in 6-epsilon dimensions, Physical Review Letters 71 (1993), p. 3222.

[65] O. Perković, K. Dahmen, and J.P. Sethna, Avalanches, Barkhausen Noise, and Plain Old Criticality, Physical Review Letters 75 (1995), p. 4528.

[66] K. Dahmen and J.P. Sethna, Hysteresis, avalanches, and disorder-induced critical scaling: A renormalization-group approach, Physical Review B 53 (1996), p. 14872.

[67] O. Perković, K.A. Dahmen, and J.P. Sethna, Disorder-induced critical phenomena in hysteresis: Numerical scaling in three and higher dimensions, Physical Review B 59 (1999), p. 6106.

[68] A. Travesset, R.A. White, and K.A. Dahmen, Crackling noise, power spectra, and disorderinduced critical scaling, Physical Review B 66 (2002), p. 024430.

[69] J.H. Carpenter and K.A. Dahmen, Barkhausen noise and critical scaling in the demagnetization curve, Physical Review B 67 (2003), p. 020412.

[70] H. Ji and M.O. Robbins, Percolative, self-affine, and faceted domain growth in random threedimensional magnets, Physical Review B 46 (1992), pp. 14519 - 14527.

[71] A.P. Mehta et al., Universal pulse shape scaling function and exponents: Critical test for avalanche models applied to Barkhausen noise, Physical Review E 65 (2002), p. 046139.

[72] E. Vives and A. Planes, Avalanches in a fluctuationless first-order phase transition in a random-bond Ising model, Physical Review B 50 (1994), p. 3839.

[73] E. Vives and A. Planes, Hysteresis and avalanches in disordered systems, Journal of Magnetism and Magnetic Materials 221 (2000), pp. 164-171.

[74] L. Néel, Theory of the Lord Rayleigh magnetization law, 1, Cahiers de Phys. 12 (1942), pp. $1-20$.

[75] L. Néel, Theory of the Lord Rayleigh magnetization law, 2, Cahiers de Phys. 13 (1943), pp. $18-30$. 
[76] H.J. Williams, W. Shockley, and C. Kittel, Studies of the Propagation Velocity of a Ferromagnetic Domain Boundary, Physical Review 80 (1950), p. 1090.

[77] J. Porteseil and R. Vergne, Some new aspects of an old problem: The magnetization of a polycrystal, J. Phys.(Paris) 40 (1979), pp. 871-881.

[78] R. Vergne, J. Cotillard, and J. Portesei, Some statistical aspects of the magnetisation processes in ferromagnetic materials. A case of the displacement of a single 180 degrees Bloch wall in a monocrystalline medium with random imperfections, Revue de Physique Applique 16 (1981), pp. 449-476.

[79] P. Cizeau et al., Dynamics of a Ferromagnetic Domain Wall and the Barkhausen Effect, Physical Review Letters 79 (1997), p. 4669.

[80] G. Durin, C. Beatrice, and G. Bertotti, Microstructural study of amorphous magnetic materials through the Barkhausen effect, IEEE Transactions on Magnetics 30 (1994), pp. 464-466.

[81] G. Durin, A. Magni, and G. Bertotti, Measurements of the Barkhausen effect in FeCoB amorphous alloys, Journal of Magnetism and Magnetic Materials 160 (1996), pp. 299-301.

[82] J.R. Petta, M.B. Weissman, and K.P. O'Brien, Multiple magnetization paths in Barkhausen noise, Physical Review E 54 (1996), p. R1029.

[83] J.R. Petta, M.B. Weissman, and G. Durin, Dependence of Barkhausen pattern reproducibility on hysteresis loop size, Physical Review E 56 (1997), pp. 2776-2780.

[84] J.P. Stokes et al., Interfacial Stability of Immiscible Displacement in a Porous Medium, Physical Review Letters 57 (1986), p. 1718.

[85] M. Cieplak and M.O. Robbins, Dynamical Transition in Quasistatic Fluid Invasion in Porous Media, Physical Review Letters 60 (1988), pp. 2042 - 2045.

[86] M. Cieplak and M.O. Robbins, Influence of contact angle on quasistatic fluid invasion of porous media, Physical Review B 41 (1990), pp. 11508 - 11521.

[87] O. Narayan and D.S. Fisher, Threshold critical dynamics of driven interfaces in random media, Physical Review B 48 (1993), p. 7030.

[88] M. Alava, M. Dubé, and M. Rost, Imbibition in disordered media, Advances in Physics 53 (2004), pp. 83-175.

[89] G. Blatter et al., Vortices in high-temperature superconductors, Reviews of Modern Physics 66 (1994), p. 1125.

[90] D.S. Fisher, Sliding charge-density waves as a dynamic critical phenomenon, Physical Review B 31 (1985), p. 1396.

[91] G. Grüner, The dynamics of charge-density waves, Reviews of Modern Physics 60 (1988), p. 1129.

[92] O. Narayan and D.S. Fisher, Critical behavior of sliding charge-density waves in 4- epsilon dimensions, Physical Review B 46 (1992), p. 11520.

[93] D.S. Fisher et al., Statistics of Earthquakes in Simple Models of Heterogeneous Faults, Physical Review Letters 78 (1997), p. 4885.

[94] M. Alava et al., Statistical models of fracture, Advances in Physics 55 (2006), pp. 349 - 476.

[95] J. Koivisto, M. Rost, and M. Alava, Creep of a Fracture Line in Paper Peeling, Physical Review Letters 99 (2007), p. 145504.

[96] M. Kardar, Nonequilibrium dynamics of interfaces and lines, Physics Reports 301 (1998), pp. $85-112$.

[97] F.D. S, Collective transport in random media: from superconductors to earthquakes, Physics reports 301 (1998), pp. 113-150.

[98] J.S. Urbach, R.C. Madison, and J.T. Markert, Interface Depinning, Self-Organized Criticality, and the Barkhausen Effect, Physical Review Letters 75 (1995), p. 276.

[99] O. Narayan, Self-Similar Barkhausen Noise in Magnetic Domain Wall Motion, Physical Review Letters 77 (1996), p. 3855.

[100] J.K. Galt, Motion of Individual Domain Walls in a Nickel-Iron Ferrite, Bell Syst. Tech. J. 33 (1954), p. 1023.

[101] C.P. Bean and D.S. Rodbell, Influence of Pulsed Magnetic Fields on the Reversal of Magnetization in Square Loop Metallic Tapes, Journal of Appl. Phys. 26 (1955), p. 1318.

[102] D.S. Rodbell and C.P. Bean, Some Properties of the Coercive Force in Soft Magnetic Materials, Physical Review 103 (1956), pp. 886-895.

[103] O. Narayan and D.S. Fisher, Dynamics of sliding charge-density waves in 4- epsilon dimensions, Physical Review Letters 68 (1992), p. 3615.

[104] A.I. Larkin and D.E. Khmel'nitskii, Phase transition in uniaxial ferroelettrics, Zh. Eksp. teor. Fiz. 56 (1969), pp. 2087-2098 [Soviet Physics JETP, 29, 1123 (1969)], reprinted in 30 Years of the Landau Institute: Selected Papers Di Isaak Markovich Khalatnikov, Vladimir P. Mineev, 
World Scientific, 1996, pg. 43.

[105] A.A. Fedorenko and S. Stepanow, Depinning transition at the upper critical dimension, Physical Review Letters 67 (2003), p. 057104.

[106] D.H. Kim, S.B. Choe, and S.C. Shin, Direct Observation of Barkhausen Avalanche in Co Thin Films, Physical Review Letters 90 (2003), p. 087203.

[107] D.H. Kim, S.B. Choe, and S.C. Shin, Time-resolved observation of Barkhausen avalanche in Co thin films using magneto-optical microscope magnetometer, Journal of Appl. Phys. 93 (2003), p. 6564.

[108] H. Leschhorn et al., Driven interface depinning in a disordered medium, Annalen der Physik 6 (1997), pp. 1-34.

[109] G. Durin and S. Zapperi, Barkhausen noise in soft amorphous magnetic materials under applied stress, J. Appl. Phys. 85 (1999), pp. 5196-5198.

[110] M. Bahiana et al., Domain size effects in Barkhausen noise, Physical Review E 59 (1999), p. 3884 .

[111] T. Nattermann et al., Dynamics of interface depinning in a disordered medium, Journal de Physique II France 2 (1992), pp. 1483-1488.

[112] G. Durin and S. Zapperi, Complex dynamics of magnetic domain walls, Physica A: Statistical Mechanics and its Applications 314 (2002), pp. 230-234.

[113] F. Colaiori, S. Zapperi, and G. Durin, Shape of a Barkhausen pulse, Journal of Magnetism and Magnetic Materials 272-276 (2004), pp. E533-E534.

[114] A. Baldassarri, F. Colaiori, and C. Castellano, Average Shape of a Fluctuation: Universality in Excursions of Stochastic Processes, Physical Review Letters 90 (2003), p. 060601.

[115] F. Colaiori, A. Baldassarri, and C. Castellano, Average trajectory of returning walks, Physical Review E 69 (2004), p. 041105.

[116] S. Redner A Guide to First-passage Processes, Cambridge University Press, United Kingdom, 2001.

[117] G. Durin and S. Zapperi, On the power spectrum of magnetization noise, Journal of Magnetism and Magnetic Materials 242-245 (2002), pp. 1085-1088.

[118] G. Durin et al., Signature of negative domain wall mass in soft magnetic materials, Journal of Magnetism and Magnetic Materials 316 (2007), pp. 436-441.

[119] S. Zapperi et al., Signature of effective mass in crackling-noise asymmetry, Nature Physics 1 (2005), pp. 46-49.

[120] W. Döering, Ferromagnetic Resonance, Z. Naturforschung 3a (1948), p. 373.

[121] R. Becker, La dynamique de la paroi de Bloch et la perméabilité en haute fréquence, Journal de Physique et le Radium 12 (1951), pp. 332-338.

[122] C. Kittel and J. Gait, Magnetic Structure of Ferromagnets, Solid State Physics 3 (1956), p. 437.

[123] J.E.L. Bishop, The contribution made by eddy currents to the effective mass of a magnetic domain wall, Journal of Physics D: Applied Physics 13 (1980), pp. L15-L19.

[124] F. Colaiori, G. Durin, and S. Zapperi, Eddy current damping of a moving domain wall: Beyond the quasistatic approximation, Physical Review B 76 (2007), pp. 224416-7.

[125] B. Miller, C. O'Hern, and R.P. Behringer, Stress Fluctuations for Continuously Sheared Granular Materials, Physical Review Letters 77 (1996), p. 3110.

[126] F. Dalton and D. Corcoran, Basin of attraction of a bounded self-organized critical state, Physical Review E 65 (2002), p. 031310.

[127] B. Gutenberg and C.F. Richter Seismicity of the Earth and Associated Phenomena, Princeton University Press, Princeton, N. J., 1949.

[128] International Handbook of Earthquake and Engineering Seismology: Part A, Academic Press, Amsterdam, 2002 ed William H.K. Lee and Hiroo Kanamori and Paul Jennings and Carl Kisslinger and William Hung Kan Lee.

[129] F. Omori, On the aftershocks of earthquakes, Journal of the College of Science, Imperial University of Tokyo 7 (1894), pp. 111-200.

[130] C. Kisslinger and L.M. Jones, Properties of aftershock sequences in southern California, Journal of Geophysical Research 96 (1991), pp. 11947-11958.

[131] Y. Ben-Zion and J.R. Rice, Earthquake failure sequences along a cellular fault zone in a threedimensional elastic solid containing asperity and nonasperity regions, Journal of Geophysical Research 98 (1993), pp. 14109-14132.

[132] K. Dahmen, Nonlinear dynamics: Universal clues in noisy skews, Nat Phys 1 (2005), pp. 13-14.

[133] Y. Ben-Zion and J.R. Rice, Slip patterns and earthquake populations along different classes of faults in elastic solids, Journal of Geophysical Research 100 (1995), p. 12956. 
[134] Y. Ben-Zion, Stress, slip, and earthquakes in models of complex single-fault systems incorporating brittle and creep deformations, Journal of Geophysical Research 101 (1996), pp. 5677-5706.

[135] P. Bak, C. Tang, and K. Wiesenfeld, Self-organized criticality: An explanation of the $1 / f$ noise, Physical Review Letters 59 (1987), pp. $381-384$.

[136] F..L. T . Richeton J . Weiss, Dislocation avalanches: Role of temperature, grain size and strain hardening, Acta Materialia 53 (2005), pp. $4463-4471$.

[137] L. Laurson and M.J. Alava, $1 / f$ noise and avalanche scaling in plastic deformation, Physical Review E 74 (2006), p. 066106.

[138] F.J. Pérez-Reche, L. Truskinovsky, and G. Zanzotto, Training-Induced Criticality in Martensites, Physical review Letters 99 (2007), p. 075501. 